\title{
MicroED Elucidation of Diverse Solid-State Packing in a Family of Electron-Deficient Expanded Helicenes
}

\author{
Adrian E. Samkian, ${ }^{[a], \dagger}$ Gavin R. Kiel, ${ }^{[a],+}$ Christopher G. Jones, ${ }^{[b]}$ Harrison M. Bergman, ${ }^{[a]}$ Julia \\ Oktawiec, ${ }^{[a]}$ Hosea M. Nelson, ${ }^{[[b]}$ and T. Don Tilley ${ }^{*[a]}$
}

[a] Department of Chemistry, University of California, Berkeley, Berkeley, California, 94720, United States

[b] Department of Chemistry and Biochemistry, University of California, Los Angeles, Los Angeles, California, 90095, United States

†Denotes equal contribution

\begin{abstract}
Solid-state packing plays a defining role in the properties of a molecular organic material, but it is difficult to elucidate in the absence of single crystals that are suitable for X-ray diffraction. Here, we demonstrate the coupling of divergent synthesis with microcrystal electron diffraction (MicroED) for rapid assessment of solid-state packing motifs, using a class of chiral nanocarbons - expanded helicenes - as a proof of concept. Two highly selective oxidative dearomatizations of a readily-accessible helicene provided a divergent route to four electron-deficient analogues containing quinone or quinoxaline units. Crystallization efforts consistently yielded microcrystals that were unsuitable for single crystal X-ray diffraction, but ideal for MicroED. This technique facilitated the elucidation of solid-state structures of all five compounds with $<1.1 \AA$ resolution. The otherwise-inaccessible data revealed a range of notable packing behavior, including four different space groups, homochirality in a crystal for a helicene with an extremely low enantiomerization barrier, and nanometer scale cavities. The results of this study suggest that MicroED will soon become an indispensable tool for high-throughput investigations in pursuit of next-generation organic materials.
\end{abstract}

\section{Introduction}

There is growing interest in chirality as an orthogonal design element for next-generation molecular organic materials. ${ }^{1}$ This parameter gives rise to new properties as a result of its impact on electronic structure, including circularly polarized absorption and emission, ${ }^{2}$ nonlinear optical behavior, ${ }^{3}$ and spin polarization. ${ }^{4}$ While the impact of chirality on molecular properties is important, its impact on supramolecular and solid-state behavior is especially relevant if the molecule is to be integrated into a material or device. ${ }^{5}$ The low symmetry and diverse geometries of chiral molecules have profound implications for the properties of their aggregates, but the complexities of such structures severely complicate efforts toward rational design. ${ }^{6}$

As the prototypical chiral aromatic molecules, helicenes helical arrays of fused aromatic rings - have been at the forefront of efforts to exploit chirality in organic (opto)electronic applications. ${ }^{1 d-f}$ The structural diversity of these compounds has recently grown at a rapid rate, with a particular surge of structures containing multiple helicenes embedded into larger polyaromatic frameworks. ${ }^{7}$ Helicenes exhibit remarkable supramolecular and solid-state chemistry promoted by m-stacking interactions, which can result in enhanced or emergent properties. ${ }^{3,7 c, 8}$ For example, using a versatile helicene quinone scaffold, Nuckolls, Katz, and Verbiest demonstrated significant enhancements of specific rotation, circular dichroism, and second-order nonlinear optical susceptibility due to aggregation. ${ }^{3,8 a}$ More recently, Itami and coworkers reported a double helicene that exhibits m-stacking in all three crystallographic dimensions, a rare phenomenon that may give rise to isotropic charge transport. ${ }^{7 c}$ Along these lines, the unique solid-state behavior of helicenes has been exploited in a device setting. For example, in an organic field effect transistor, the enantiopurity of the helicene-based active layer can have dramatic or unexpected effects on performance..$^{2 \mathrm{~b}, 8 \mathrm{~b}-\mathrm{c}}$ Nakamura demonstrated that a charge carrier inversion (switch from $\mathrm{p}$ - to $\mathrm{n}$ type) can occur on going from a racemic to an enantiopure helicene ${ }^{8 b}$ and Fuchter observed an 80-fold increase in charge carrier mobility for a racemic active layer (vs. an enantiopure analogue). ${ }^{8 c}$ These results motivate further research related to helicene solid-state structure and highlight the important role of chirality.

In 2017, the Tilley group introduced a new class of chiral nanocarbons, "expanded helicenes," which possess internal cavities and large diameters due to alternating angular and linear ring fusion (e.g. 1a-b, Figure 1a). ${ }^{9}$ One exciting feature of these compounds is their unique self-assembly and solid-state packing, facilitated in part by their structural flexibility. For example, expanded [13]helicene $\mathbf{1 b}$ dimerizes via $\pi$-stacking to form the unusual double helix 1b-Dim, both in chloroform- $d$ solution and in the solid-state, which is accompanied by a large $(>2 x)$ increase in the pitch of the constituent helicenes. ${ }^{9 a}$ In contrast, no aggregation was apparent for the [11]helicene analogue (1a). Subsequently, the covalent linkage of two expanded helicenes afforded a configurationally labile "figure-eight" dimer that crystallized into an intricate homochiral network containing two distinct yet interconnected helical superstructures. ${ }^{9 c}$

Single crystal X-ray diffraction is the gold standard for solidstate structure elucidation since it can provide structural parameters with a high level of precision; however, even for crystalline compounds, it can be difficult or impossible to obtain suitable single crystals for analysis, which can preclude valuable insights. For example, our inability to obtain suitable crystals of $\mathbf{1 a}$ prevented comparisons with its apparently more interesting analogue $\mathbf{1 b} .^{9 a}$ Microcrystal electron diffraction (MicroED) has recently emerged as a way to circumvent limitations related to crystal growth since it can allow high-resolution structural data to be acquired on nanocrystalline samples. ${ }^{10}$

In this contribution, we use a combination of divergent synthesis and MicroED to interrogate solid-state behavior in a new family of expanded helicenes. The quinone- and quinoxilinecontaining helicenes 2-mon, 2-di, 3-mon, and 3-di (Scheme 1b) were targeted due to their electron deficiency, which tends to promote strong intermolecular interactions between $\pi$ systems. ${ }^{8 a, 11}$ The new compounds were accessed via a highly selective oxidative dearomatization of electron-rich 1a, which provided derivatives of the same or reduced symmetry. While uncontrollable crystallization kinetics consisitently resulted in the 
formation of microcrystals that were unsuitable for single crystal $X$-ray diffraction, the application of MicroED enabled the rapid acquisition of high resolution data for all five helicenes. This data revealed three different packing motifs, representing four different space groups. Three of the structures feature large $(1.3-2.0 \mathrm{~nm})$ channels, enforced by the ability of the helicenes to accommodate large distortions in molecular geometry.
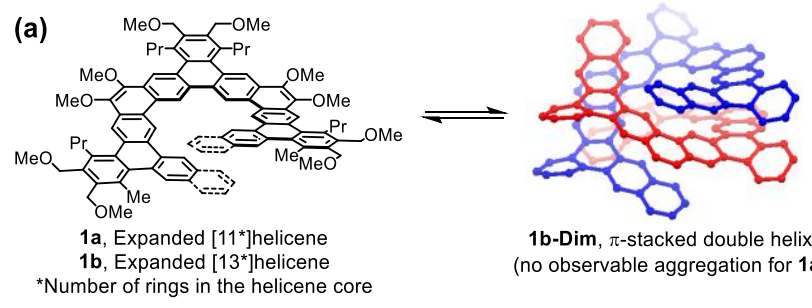

1b-Dim, $\pi$-stacked double helix (no observable aggregation for $1 \mathbf{a}$ )

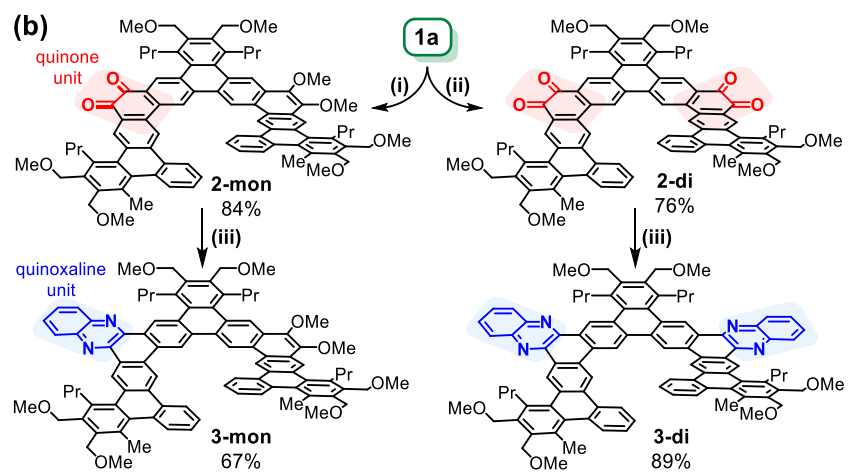

Scheme 1. (a) Previous Work: Expanded [11]- and [13]helicenes 1a and 1b, and the observation of $\pi$-stacked double helix $\mathbf{1 b}$-Dim (both in solution and the solid-state). Compound 1a could not be analyzed by X-ray crystallography due to the difficulty in obtaining suitable single crystals; (b) This work: A simple post-synthetic oxidation of electron-rich and non-aggregating 1a provided rapid, divergent access to electron deficient analogues 2-di and 3-di and donoracceptor analogues 2-mon and 3-mon. Conditions: (i) $\mathrm{CAN}$ (6 equiv), $\mathrm{CH}_{2} \mathrm{Cl}_{2}$, $\mathrm{MeCN}, \mathrm{H}_{2} \mathrm{O}, 22{ }^{\circ} \mathrm{C}$; (ii) $\mathrm{CAN}$ (15 equiv), $\mathrm{CH}_{2} \mathrm{Cl}_{2}, \mathrm{MeCN}, \mathrm{H}_{2} \mathrm{O}, 22{ }^{\circ} \mathrm{C}$; (iii) 1,2 diaminobenzene ( 5 and 20 equiv for 3 -mon and 3 -di, respectively), DCE, $70^{\circ} \mathrm{C}$.

\section{Results and Discussion}

Quinone-containing helicenes display desirable properties due to their low LUMO levels, including red-shifted absorption, reversible redox behavior, and strong aggregation. ${ }^{8 \mathrm{a}, 12}$ Furthermore, polycyclic aromatic hydrocarbons (PAHs) with quinone functionalities are well-established synthons for a range of derivatives, including $\pi$-extended cyclopentadienones, ${ }^{13}$ imidazoles, ${ }^{14}$ and pyrazines, ${ }^{15}$ which facilitates divergent manipulation of properties. ${ }^{16}$ An expedient way to install quinone units is via the oxidation of a PAH with a 1,2-dimethoxy substitution pattern. ${ }^{16 a, 17}$ Since compound $1 \mathrm{a}$ is available using a scalable procedure, ${ }^{9 a}$ this strategy seemed to represent an opportunity to significantly increase functional diversity in expanded helicenes. Thus, in an attempt to fully oxidize 1a to its diquinone derivative 2-di (Scheme 1), this compound was treated with an excess ( 6 equiv) of cerium ammonium nitrate (CAN) in a $\mathrm{CH}_{2} \mathrm{Cl}_{2} / \mathrm{MeCN} / \mathrm{H}_{2} \mathrm{O}$ solvent system. Remarkably, this instead resulted in the selective formation of monoquinone 2-mon, isolated in $84 \%$ yield. The exceptional selectivity for 2-mon over 2-di is surprising given the highly benzenoid nature of $\mathbf{1 a}$ and its quinone derivatives, which is expected to limit electronic communication across the $\pi$-system. ${ }^{18}$ This suggests that inductive effects play an important role in slowing down the oxidation of the second 1,2-dimethoxyarene unit even though the two reaction sites are physically distant. Importantly, diquinone 2di was also accessed, in $76 \%$ yield, simply with use of a larger excess of CAN (15 equiv).

The condensation of a 1,2-diamine onto a quinonecontaining $\mathrm{PAH}$ is a reliable and general transformation that provides a means to install functionally-valuable nitrogen atoms and extend the $\pi$-system, ${ }^{15}$ which could lead to stronger $\pi$ stacking interactions between molecules. ${ }^{11}$ Thus, 2-mon and 2-di were treated with excess 1,2-phenylenediamine in 1,2dichloroethane (DCE) at $70{ }^{\circ} \mathrm{C}$, which afforded quinoxalinecontaining helicenes 3-mon and 3-di in 67\% and $89 \%$ isolated yields, respectively, after a simple workup/purification procedure consisting of precipitation followed by recrystallization from toluene. The identities of all new helicenes were initially confirmed with ${ }^{1} \mathrm{H}$ and ${ }^{13} \mathrm{C}$ NMR spectroscopies and high-resolution mass spectrometry (HRMS-ESI). Furthermore, the structures for all five helicenes were unambiguously confirmed by MicroED (see below).

The substitution of PAHs with quinone units often results in solubility problems, ${ }^{17 b, 19}$ but the new helicenes 2-mon and 2-di are highly soluble $\left(>100 \mathrm{mg} / \mathrm{mL}\right.$ in $\mathrm{CHCl}_{3}$ and $\mathrm{CH}_{2} \mathrm{Cl}_{2}$ ). This is especially notable given their large size (14 fused rings). The solubility may be attributed to two different forms of non-planarity: 1) the helical distortion that is present in all helicenes and 2) a bending of the benzannulated rings away from the contour of the helix, which results from alkyl substituents in six different bay positions. While the quinoxaline-annulated helicenes 3-mon and 3-di are similarly soluble to the quinone analogs in a thermodynamic sense, their kinetic solubility is low when isolated as a crystalline solid.

The photophysical properties of the new helicenes were probed by UV/Vis absorption and emission spectroscopies (Figure 1a-b). Most notable are the sizable decreases in photophysical HOMO-LUMO gaps (calculated from their absorption onset values) for quinone-containing helicenes 2-mon $(2.08 \mathrm{eV})$ and 2-di $(2.05 \mathrm{eV})$ relative to that for their synthetic precursor $1 \mathrm{a}(2.79 \mathrm{eV})$. In contrast, the quinoxaline units have only a small effect on this value $(2.63 \mathrm{eV}$ for both 3-mon and 3di). Relative to the absorption maxima for $\mathbf{1 a}(324 \mathrm{~nm})$, small red shifts of 9 and $15 \mathrm{~nm}$ are observed for the mono- and diquinones 2-mon and 2-di, respectively, whereas blue shifts of comparable magnitude (5 and $9 \mathrm{~nm}$ ) are observed for the mono- and diquinoxalines 3-mon and 3-di. Despite limited perturbation of their absorption maxima and onset values, 3-mon and 3-di display relatively large bathochromic shifts in emission maxima relative to that for 1a (from $446 \mathrm{~nm}$ to 533 and $517 \mathrm{~nm}$, respectively). As is typical for PAHs containing quinone units, ${ }^{20} 2$ mon and 2-di are non-emissive. 

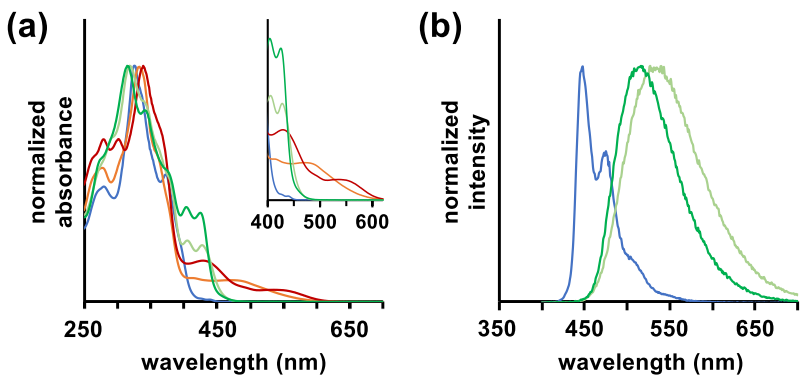

(c)

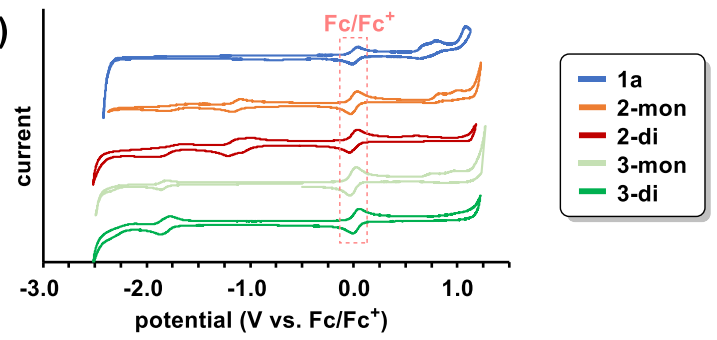

Figure 1. UV/Vis (a) absorption and (b) emission spectra in $\mathrm{CH}_{2} \mathrm{Cl}_{2}$; (c) Cyclic voltammograms in $\mathrm{CH}_{2} \mathrm{Cl}_{2}\left(1.0 \mathrm{mM}\right.$ ) with $0.1 \mathrm{M}^{n} \mathrm{Bu}_{4} \mathrm{NPF}_{6}$ as the supporting electrolyte, scanned at $100 \mathrm{mV} / \mathrm{s}$.

The electrochemical behavior of the helicenes was investigated by cyclic voltammetry $(\mathrm{CV})$ in $\mathrm{CH}_{2} \mathrm{Cl}_{2}$ solution (Figure 1c). Only 1a, 2-mon, and 3-mon display oxidation events (one per 1,2-dimethoxy substituent), which were quasi-reversible or irreversible. The four new compounds with quinone or quinoxaline units display one reversible reduction event per unit (e.g. the presence of two quinones in 2-di gives rise to two reversible reduction events). ${ }^{21}$ The electrochemical HOMO-LUMO gaps for 2-mon and 3-mon (1.94 and $2.57 \mathrm{eV}$, respectively) are slightly smaller than those calculated from the absorption onset (see above). Replacement of the 1,2-dimethoxyarene units with quinone or quinoxaline units does not significantly affect $\mathrm{HOMO}$ energy levels. Compared to the HOMO level for 1a $(-5.46 \mathrm{eV})$, only slight decreases were observed for each additional quinone $(\sim 0.15 \mathrm{eV})$ or quinoxaline $(\sim 0.06 \mathrm{eV})$ unit. The large changes in $\mathrm{HOMO} / \mathrm{LUMO}$ gaps result primarily from changes in LUMO levels $(\sim 1.0 \mathrm{eV}$ decreases for 2-mon and 2-di and $\sim 0.3 \mathrm{eV}$ decreases for 3-mon and 3-di).

One of the primary motivations for structural modifications of compound 1a was its limited aggregation via m-stacking. Aggregation behavior was initially probed by variable concentration ${ }^{1} \mathrm{H}$ NMR spectroscopy in chloroform- $d$ solution. Like its precursor 1a, monoquinone 2-mon does not appear to aggregate to an appreciable extent in this solvent, as evidenced by the invariance of its ${ }^{1} \mathrm{H}$ NMR spectrum to concentration (Figures S9 and S10). In contrast, the ${ }^{1} \mathrm{H}$ NMR spectra of 2-di, 3mon, and 3-di all exhibit concentration dependence (Figure 2). The most dramatic effects are observed for 3-di, whose chemical shifts are shielded by up to $1.3 \mathrm{ppm}$ upon concentration from $0.024 \mathrm{mM}$ to $80 \mathrm{mM}$. In contrast, the chemical shifts of monoquinoxaline 3-mon and diquinone 2-di exhibit a combination of shielding and de-shielding, and of a smaller magnitude. Compounds 2-di, 3-mon, and 3-di are rare in that they simultaneously aggregate and exhibit high solubility, without the need for long alkyl chains on the periphery. Since these two properties are usually at odds with one another, and since both are of critical importance for applications (e.g. in organic electronics), further elucidation of the nature of the aggregation was of interest.
Dynamic light scattering (DLS) experiments were conducted to probe aggregate size in chloroform solution (Figure S24). Measurements were made for diquinone 2-di and diquinoxaline 3-di since their variable concentration ${ }^{1} \mathrm{H}$ NMR spectra suggest notable aggregation, and the non-aggregating parent helicene 1a was analyzed for comparison. As expected, the DLS measurements suggest that $\mathbf{1 a}$ is monomeric regardless of concentration, at an average size of $\sim 1.0 \mathrm{~nm} .{ }^{22}$ In contrast, the size of 2-di exhibits strong concentration dependence, from 1.0 $\mathrm{nm}$ at $0.24 \mathrm{mM}$ (consistent with monomeric 2-di) to $271 \mathrm{~nm}$ at 24 $\mathrm{mM}$ (consistent with supramolecular polymers). For diquinoxaline 3-di, the size approaches $1.7 \mathrm{~nm}$ at high concentration, which suggests that the large upfield shifts observed in the ${ }^{1} \mathrm{H}$ NMR spectra of concentrated solutions result from small aggregates.

The observation of small aggregates for 3-di via DLS prompted the use of the Horman/Dreux model for quantitative assessment of dimeric aggregation equilibria. ${ }^{23}$ Using the aromatic chemical shifts from the variable concentration ${ }^{1} \mathrm{H}$ NMR spectra, this model provided an excellent fit and an average association constant for dimerization $\left(K_{\mathrm{a}}\right)$ of $13 \mathrm{M}^{-1}$ at $298 \mathrm{~K}$. Thus, these data are consistent with those from DLS, suggesting that higher-order aggregates of 3-di do not play a significant role in the concentration range studied. The $K_{a}$ value is slightly lower than that for expanded [13]helicene 1b $\left(23 \mathrm{M}^{-1}\right){ }^{9 a}$ but these values may not be directly comparable since $\mathbf{1 b}$ exhibits the welldefined monomer-dimer equilibrium shown in Figure 1a whereas 3-di may aggregate differently.

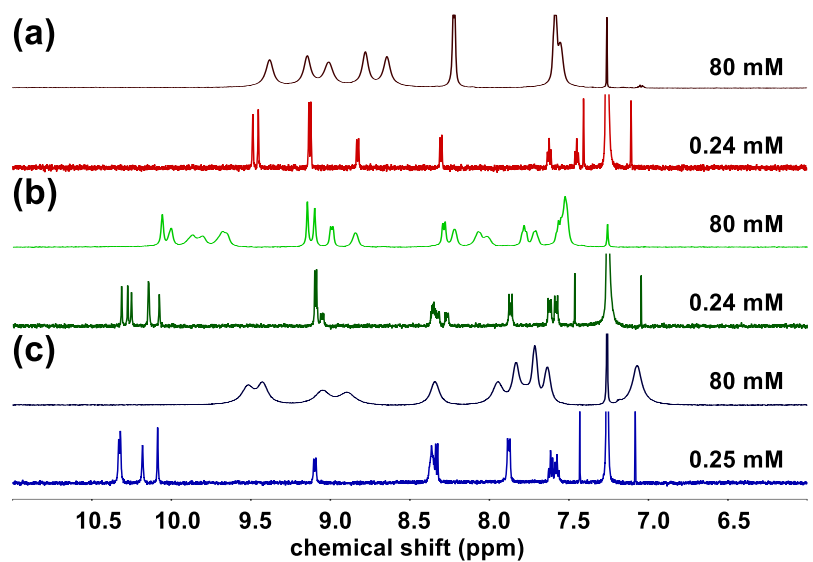

Figure 2. Variable concentration partial ${ }^{1} \mathrm{H}$ NMR spectra of helicenes (a) 2-di, (b) 3-mon and (c) 3-di in chloroform-d solution. The analogous spectra for helicenes 1a and 2-mon are invariant to concentration (See SI).

Previously obtained crystal structures of expanded helicenes have revealed unique packing architectures and provided insight into their supramolecular interactions. ${ }^{9}$ In pursuit of this information, significant effort was devoted to growing single crystals suitable for X-ray diffraction, but these efforts consistently provided microcrystals ${ }^{24}$ that were unsuitable (even with the aid of synchrotron radiation) for all compounds except 3-mon. Thus, we turned to MicroED, and with this technique the structures of all five helicenes were elucidated with ease (Figure 3). The crystals used for analysis were grown as follows. For 1a, the bulk, microcrystalline material obtained during its synthesis proved to be of sufficient quality. For 2-mon and 2-di, diffusion of pentane into an EtOAc solution yielded orange needles. For 3-mon and 3di, slow evaporation of a benzene solution provided yellow rods. 
(a) $1 \mathrm{a}$

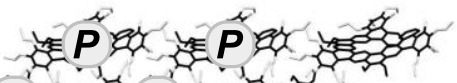

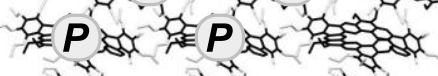
Les

o 30 o ror

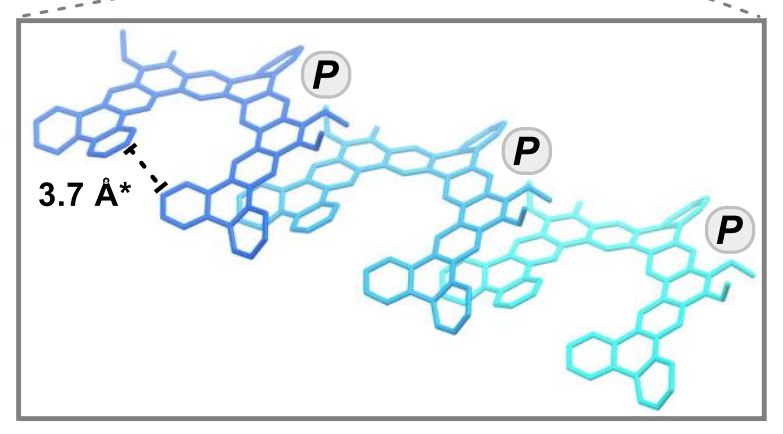

(b) 2-di

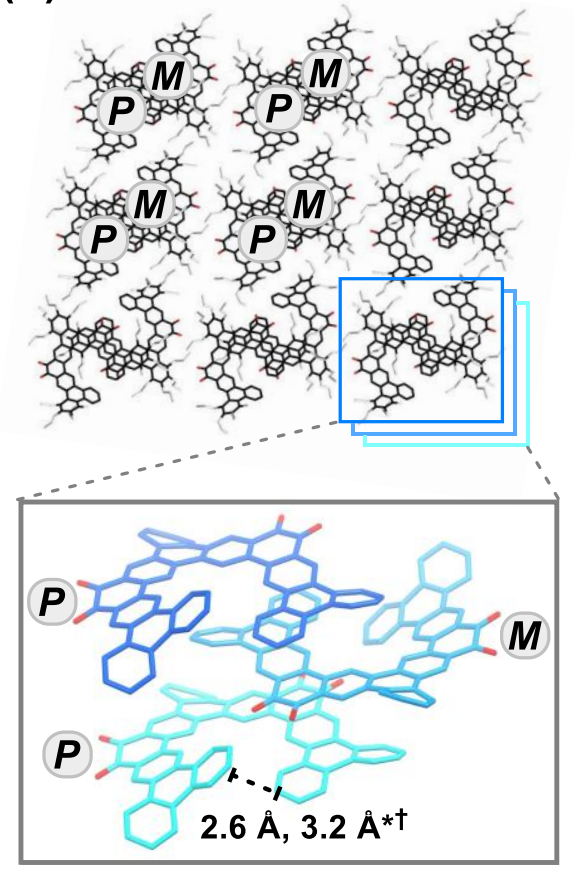

(c) 2-mon
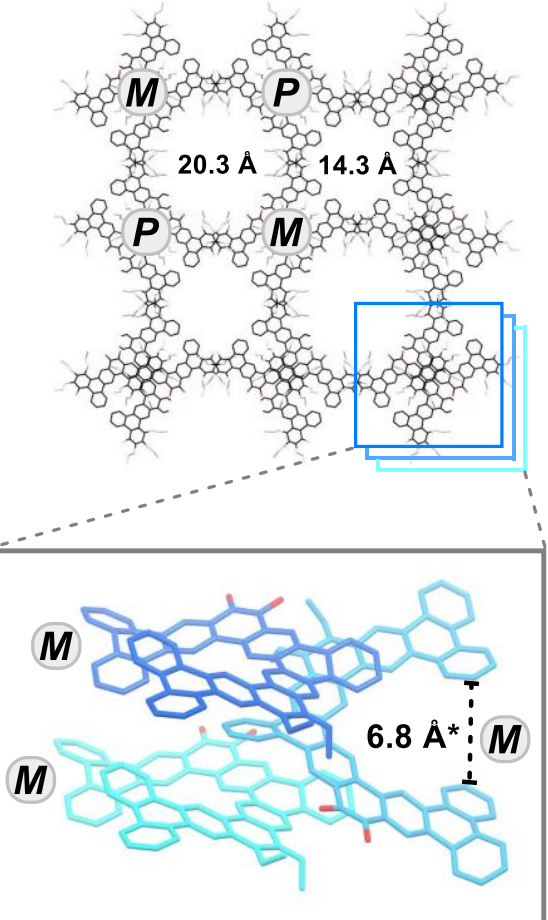

(d) 3-mon
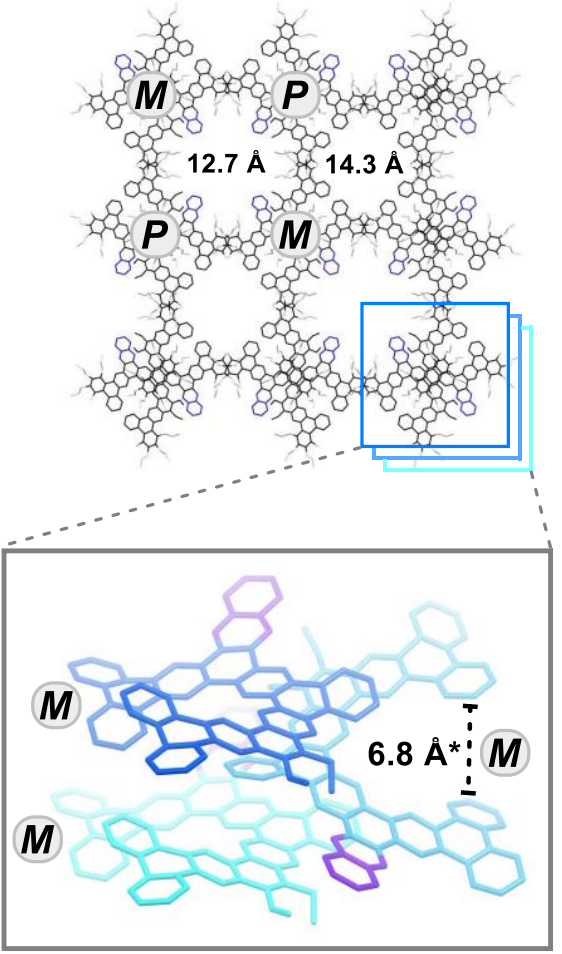

(e) 3-di
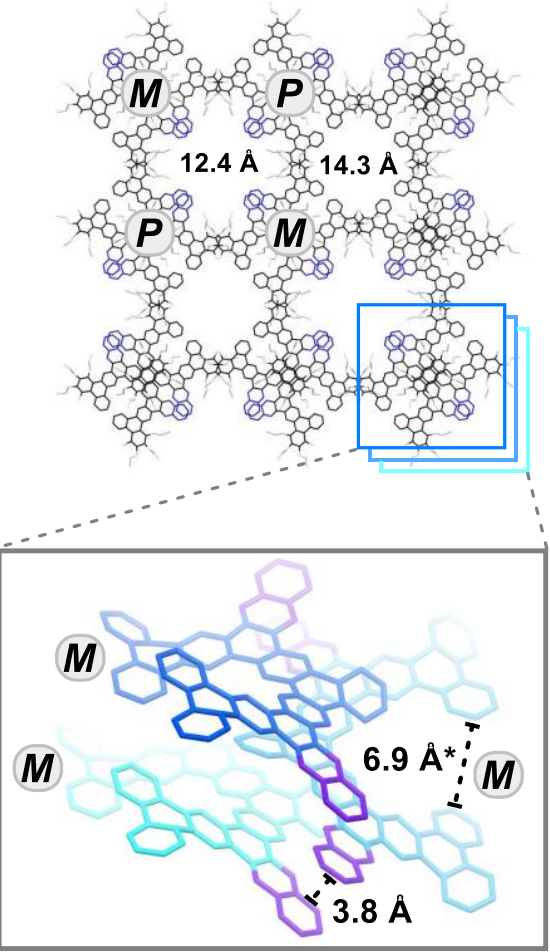

Figure 3. Solid-state MicroED structures of (a) 1a, (b) 2-di, (c) 2-mon, (d) 3-mon, and (e) 3-di. *The starred distances represent helical pitch values for the associated helicene, which were estimated as described in Figure 4. ${ }^{\dagger}$ The crystals of 2-di feature helicenes with two different pitch values. 
The parent, electron-rich helicene 1a crystallized in the chiral $P 212121$ space group, wherein the constituent molecules are homochiral and form weakly bound columns (Figure 3a). This is the second example of a homochiral expanded helicene crystal structure ${ }^{9 c}$ and it is notable since 1a is expected to have an extremely low enantiomerization barrier in solution. The closest $\pi$-stacking distance is $3.8 \AA$, and there are only two overlapping rings. These weak interactions are consistent with the absence of observable aggregation in chloroform- $d$ solution. ${ }^{9 a}$ Notably, the packing is quite different than that of the isolated double helical dimers observed in the longer analogue 1b (Figure 1a), which differs by only two rings at the terminus.

While the quinone-containing helicenes 2-di and 2-mon differ from 1a only with respect to their oxidation state and associated methyl groups, all three compounds exhibit unique crystal packing. Compounds 2-di and 2-mon crystallized in the Iba2 and $P \overline{1}$ space groups, respectively (Figures $3 b$ and $3 c$ ). In contrast to that of $1 \mathrm{a}$, these space groups are achiral. All three crystal structures feature columns driven by long-range $\pi-$ stacking; however, in contrast to $\mathbf{1 a}$ and 2-mon, the columns of 2-di are heterochiral (i.e. the helicenes within a given column alternate handedness), which leads to an achiral crystal. Individual columns for 2-mon are homochiral, with alternation of chiralities between columns to make the overall structure achiral.

Compared to 1a, the molecules of both quinone-containing helicenes exhibit more pronounced intermolecular interactions. This is especially true for 2-di, which features eight overlapping rings per helicene and a closest $\pi$-contact of $3.4 \AA$. This closepacking appears to be driven by interactions between electrondeficient quinone units and relatively electron-rich benzannulated rings (two per molecule). The intermolecular interactions in 2mon support a complex framework with emergent macroscopic features, including the existence of two large cavities.

The crystal packings of 3-mon and 3-di (Figure $3 \mathrm{~d}$ and $3 \mathrm{e}$, respectively) are similar to that for 2-mon in that all three exhibit a combination of one intrinsic and two extrinsic cavities. The intrinsic cavity ( $\sim 4 \AA$ in diameter) is consistent across all compounds since it is enforced by the alternating linear and angular ring fusion pattern that defines expanded helicenes. In all three cases, such cavities are occupied by the central methoxymethyl sidechains of neighboring molecules. There are two extrinsic cavities that emerge in each structure due to the packing arrangement. One is bordered by the terminal rings of the helicenes and is identical in all three structures $(14.3 \AA$ in diameter). The other differs for each helicene, and its size is dictated by the peripheral functionality (20.3 $\AA$ for 2-mon, $12.7 \AA$ for 3-mon, $12.4 \AA$ for 3-di). Further examination of these cavities through the Fourier difference map shows little residual density, but conclusions cannot be drawn about the presence or absence of solvent molecules due to the possibility of disorder. Like 2-mon, compound 3-mon also crystallized in the Iba2 space group, but compound 3-di occupies the higher symmetry $\overline{4} c 2$ space group since it has a molecular $C_{2}$ axis (Figure $3 \mathrm{e}$ ). Each of these three helicenes has only one overlapping backbone ring with similar centroid-centroid distances (4.2 ̊̊ for 3-mon, $4.0 \AA$ for 3-di, and 4.1 $\AA$ for 2-mon) indicating weak interactions. In addition, 3-di has overlapping quinoxaline units that may contribute to its strong aggregation in solution.

A striking feature of the MicroED structures is the large helical pitches for 2-mon, 3-mon, and 3-di $(6.8,6.8$, and $6.9 \AA$, respectively). ${ }^{25}$ Previously reported expanded helicenes, and compounds 1a and 2-di described herein, have pitches in the range of 2.6-3.7 $\AA .{ }^{9}$ One exception is dimeric [13]helicene 1bDim (Figure 1a), ${ }^{9 a}$ wherein each helicene displays a large pitch $(7.4 \AA)$ to accommodate extensive $\pi$-stacking. Another case where a notable molecular distortion was observed in the crystal structure was for the recently-reported figure-eight expanded helicene dimer. ${ }^{9 c}$ There, an intricate network of $\pi$-stacking interactions appears to be the driving force for the distortion. In contrast to that for $\mathbf{1 b}$-Dim and the figure-eight helicene, the driving forces for the observed, large molecular distortions are unclear.

The distorted molecular structures of 2-mon, 3-mon, and 3di in the solid-state suggest a high level of structural flexibility for these compounds, especially given the absence of notable intermolecular interactions. This motivated a computational study in an attempt to quantify the energy costs for these distortions. First, gas phase geometry optimizations were performed using density functional theory (DFT) at the B3LYP-6311g(2d,p) level of theory (Figure 4). As expected, the calculated structures display only modest pitches of $3.9 \AA$ (2-mon), $3.9 \AA$ (3-mon), and $4.0 \AA$ (3-di), which are in much better agreement with those from previously-reported expanded helicenes (vide supra). Next, the structures were optimized with geometry constraints to approximate the pitches observed in their respective crystal structures, and the energies were compared with those from the unconstrained geometries. The energy cost for the observed molecular distortion was estimated to be $3.2,3.2$, and $3.8 \mathrm{kcal} / \mathrm{mol}$ for 2-mon, 3-mon, and 3-di, respectively (see SI for details). These relatively small values provide rationale for the remarkable ability of these helicenes to "flex" in order to accommodate novel crystal packings.
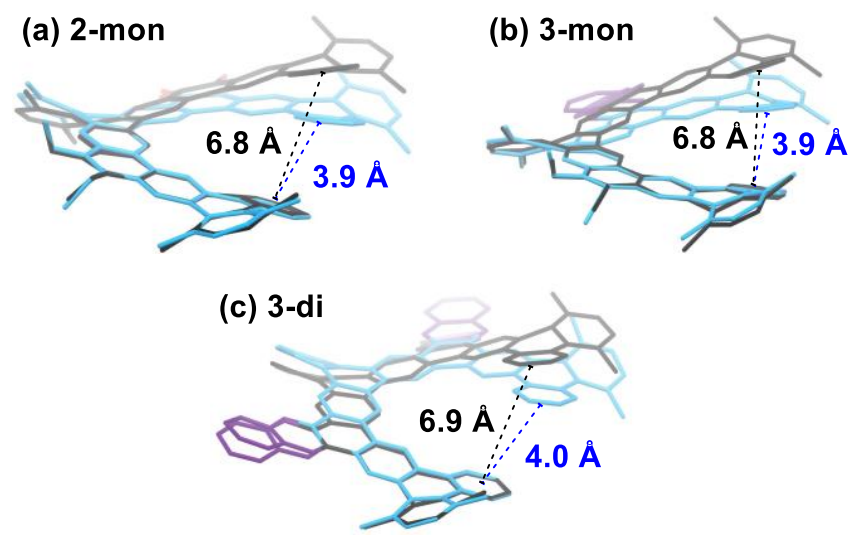

Figure 4. Comparison of MicroED (black) and calculated (blue) structures of helicenes (a) 2-mon, (b) 3-mon, and (c) 3-di. The noted distances represent the pitches of the helices that are defined by the centroids of the inner 11 rings. $^{25}$

\section{Conclusion}

In conclusion, the discovery of a selective oxidative dearomatization of a readily-available, electron-rich expanded helicene provided access to a series of four electron deficient analogues. The five helicenes displayed variations in a range of properties, but the most notable differences were related to their solid-state structures. Despite difficulties with crystal growth and the associated failures of X-ray crystallography, the acquisition of high-resolution structural data for all helicenes was easily 
accomplished with MicroED. A range of unique solid-state packing behavior was observed, including four different space groups, homochirality in a crystal for a helicene with an extremely low enantiomerization barrier, nanometer scale cavities, and large molecular distortions without an obvious driving force. The latter suggests that expanded helicene flexibility may manifest in nonintuitive ways to afford unprecedented solid-state packing. Perhaps most importantly, the results of this study provide an initiral indication of MicroED's potential utilily for high-throughput crystallization to aid the disovery of novel organic materials.

\section{Acknowledgements}

A large portion of this work was funded by the National Science Foundation under Grant No. CHE-1708210. C.G.J. acknowledges the National Science Foundation Graduate Research Fellowship Program (DGE-1650604), the Christopher S. Foote Fellowship, and the Pat Tillman Foundation for funding. J.O. acknowledges the National Science Foundation Graduate Research Fellowship Program (DGE-1650604) for funding. H.M.N. acknowledges the Packard Foundation and Bristol Myers Squibb for generous funding. A.E.S thanks Robert H. Grubbs for use of facilities and helpful discussions. The authors thank Jose Rodriguez, Duilio Cascio, Michael R. Sawaya and Michael J. Collazo (UCLA) for assistance with MicroED data collection and processing, and Vincent LaVallo, Yan Xu and William Wolf for helpful general discussions. The computational work was performed at the UC Berkeley Molecular Graphics and Computation Facility (MGCF), which is supported by the National Institute of Health (Grant No. NIH S10OD023532), and the authors thank Dave Small for his assistance with these calculations. This work incorporates data collected at the Northeastern Collaborative Access Team (NE-CAT) beamlines at Argonne National Laboratory. NE-CAT is funded by the NIHNIGMS (Grant No. P30 GM124165). The Pilatus 6M detector on the 24-ID-C beamline is funded by NIH-ORIP HEI (Grant No. S10 RR029205). This work also used resources at the Advanced Photon Source, a U.S. Department of Energy Office of Science User Facility operated by Argonne National Laboratory under Contract No. DE-AC02-06CH11357. The UCLA-DOE Institute's X-ray Crystallography Core Facility is supported by the U.S. Department of Energy (Grant No. DE-FC02-02ER63421).

\section{References}

(1) (a) Brandt, J. R.; Salerno, F.; Fuchter, M. J. The Added Value of SmallMolecule Chirality in Technological Applications. Nat. Rev. Chem. 2017 1 (6), 1-12. https://doi.org/10.1038/s41570-017-0045; (b) FernándezGarcía, J. M.; Evans, P. J.; Filippone, S.; Herranz, M. Á.; Martín, N. Chiral Molecular Carbon Nanostructures. Acc. Chem. Res. 2019, 52 (6), 15651574. https://doi.org/10.1021/acs.accounts.9b00144; (c) Rickhaus, M.; Mayor, M.; Juríček, M. Strain-Induced Helical Chirality in Polyaromatic Systems. Chem. Soc. Rev. 2016, 45, 1542-1556. https://doi.org/10.1039/C5CS00620A; (d) Shen, Y.; Chen, C.-F. Helicenes: Synthesis and Applications. Chem. Rev. 2012, 112 (3), 14631535. https://doi.org/10.1021/cr200087r; (e) Gingras, M. One Hundred Years of Helicene Chemistry. Part 1: Non-Stereoselective Syntheses of Carbohelicenes. Chem. Soc. Rev. 2013, 42 (3), 968-1006. https://doi.org/10.1039/C2CS35154D; (f) Gingras, M. One Hundred Years of Helicene Chemistry. Part 3: Applications and Properties of
Carbohelicenes. Chem. Soc. Rev. 2013, 42 (3), 1051-1095. https://doi.org/10.1039/C2CS35134J.

(2) (a) Field, J. E.; Muller, G.; Riehl, J. P.; Venkataraman, D. Circularly Polarized Luminescence from Bridged Triarylamine Helicenes. J. Am. Chem. Soc. 2003, 125 (39), 11808-11809. https://doi.org/10.1021/ja035626e; (b) Yang, Y.; da Costa, R. C.; Fuchter, M. J.; Campbell, A. J. Circularly Polarized Light Detection by a Chiral Organic Semiconductor Transistor. Nat. Photonics 2013, 7 (8), 634-638. https://doi.org/10.1038/nphoton.2013.176; (c) Tanaka, H.; Inoue, Y.; Mori, T. Circularly Polarized Luminescence and Circular Dichroisms in Small Organic Molecules: Correlation between Excitation and Emission Dissymmetry Factors. ChemPhotoChem 2018, 2 (5), 386-402. https://doi.org/10.1002/cptc.201800015.

(3) Verbiest, T.; Elshocht, S. V.; Kauranen, M.; Hellemans, L.; Snauwaert, J.; Nuckolls, C.; Katz, T. J.; Persoons, A. Strong Enhancement of Nonlinear Optical Properties Through Supramolecular Chirality. Science 1998, 282 (5390), 913-915. https://doi.org/10.1126/science.282.5390.913.

(4) Kiran, V.; Mathew, S. P.; Cohen, S. R.; Delgado, I. H.; Lacour, J.; Naaman, R. Helicenes-A New Class of Organic Spin Filter. Adv. Mater. 2016, 28 (10), 1957-1962. https://doi.org/10.1002/adma.201504725.

(5) (a) Palmans, A. R. A.; Meijer, E. W. Amplification of Chirality in Dynamic Supramolecular Aggregates. Angew. Chem. Int. Ed. 2007, 46 (47), 8948-8968. https://doi.org/10.1002/anie.200701285; (b) Yang, Y.; Zhang, Y.; Wei, Z. Supramolecular Helices: Chirality Transfer from Conjugated Molecules to Structures. Adv. Mater. 2013, 25 (42), 60396049. https://doi.org/10.1002/adma.201302448; (c) Liu, M.; Zhang, L.; Wang, T. Supramolecular Chirality in Self-Assembled Systems. Chem. Rev. 2015, 115 (15), 7304-7397. https://doi.org/10.1021/cr500671p; (d) Yashima, E.; Ousaka, N.; Taura, D.; Shimomura, K.; Ikai, T.; Maeda, K. Supramolecular Helical Systems: Helical Assemblies of Small Molecules, Foldamers, and Polymers with Chiral Amplification and Their Functions. Chem. Rev. 2016, 116 (22), 13752-13990. https://doi.org/10.1021/acs.chemrev.6b00354.

(6) Schmidt, J. A.; Weatherby, J. A.; Sugden, I.; Santana-Bonilla, A.; Salerno, F.; Fuchter, M.; Johnson, E.; Nelson, J.; Jelfs, K. Computational Screening of Organic Semiconductors: Exploring Side-Group Functionalisation and Assembly to Optimise Charge Transport in Chiral Molecules. 2020. https://doi.org/10.26434/chemrxiv.12451943.v1.

(7) (a) Barnett, L.; Ho, D. M.; Baldridge, K. K.; Pascal, R. A. The Structure of Hexabenzotriphenylene and the Problem of Overcrowded "D3h" Polycyclic Aromatic Compounds. J. Am. Chem. Soc. 1999, 121 (4), 727733. https://doi.org/10.1021/ja983471i; (b) Wang, X.-Y.; Wang, X.-C.; Narita, A.; Wagner, M.; Cao, X.-Y.; Feng, X.; Müllen, K. Synthesis, Structure, and Chiroptical Properties of a Double [7]Heterohelicene. J. Am. Chem. Soc. 2016, 138 (39), 12783-12786. https://doi.org/10.1021/jacs.6b08664; (c) Fujikawa, T.; Segawa, Y.; Itami, K. Synthesis, Structures, and Properties of $\pi$-Extended Double Helicene: A Combination of Planar and Nonplanar m-Systems. J. Am. Chem. Soc. 2015, 137 (24), 7763-7768. https://doi.org/10.1021/jacs.5b03118; (d) Pradhan, A.; Dechambenoit, P.; Bock, H.; Durola, F. Fused Helicene Chains: Towards Twisted Graphene Nanoribbons. Chem. - Eur. J. 2016, 22 (50), 18227-18235. https://doi.org/10.1002/chem.201603702; (e) Hu, Y.; Wang, X.-Y.; Peng, P.-X.; Wang, X.-C.; Cao, X.-Y.; Feng, X.; Müllen, K.; Narita, A. Benzo-Fused Double [7]Carbohelicene: Synthesis, Structures, and Physicochemical Properties. Angew. Chem. Int. Ed. 2017, 56 (12), 3374-3378. https://doi.org/10.1002/anie.201610434. (f) Hosokawa, T.; Takahashi, Y.; Matsushima, T.; Watanabe, S.; Kikkawa, S.; Azumaya, I.; Tsurusaki, A.; Kamikawa, K. Synthesis, Structures, and Properties of Hexapole Helicenes: Assembling Six [5]Helicene Substructures into Highly Twisted Aromatic Systems. J. Am. Chem. Soc. 2017, 139 (51), 18512-18521. https://doi.org/10.1021/jacs.7b07113; (g) Ball, M.; Zhong, Y.; Wu, Y.; Schenck, C.; Ng, F.; Steigerwald, M.; Xiao, S.; Nuckolls, C. Contorted Polycyclic Aromatics. Acc. Chem. Res. 2015, 48 (2), 267-276. https://doi.org/10.1021/ar500355d; (h) Li, C.; Yang, Y.; Miao, Q. Recent Progress in Chemistry of Multiple Helicenes. Chem. Asian J. 2018, 13 (8), 884-894. https://doi.org/10.1002/asia.201800073; (i) Schuster, N. J.; Paley, D. W.; Jockusch, S.; Ng, F.; Steigerwald, M. L.; Nuckolls, C. Electron Delocalization in Perylene Diimide Helicenes. 
Angew. Chem. Int. Ed. 2016, 55 (43), 13519-13523. https://doi.org/10.1002/anie.201607878; (j) Evans, P. J.; Ouyang, J.; Favereau, L.; Crassous, J.; Fernández, I.; Perles, J.; Martín, N. Synthesis of a Helical Bilayer Nanographene. Angew. Chem. Int. Ed. 2018, 57 (23) 6774-6779. https://doi.org/10.1002/anie.201800798. (k) Schuster, N. J. Joyce, L. A.; Paley, D. W.; Ng, F.; Steigerwald, M. L.; Nuckolls, C. The Structural Origins of Intense Circular Dichroism in a Waggling Helicene Nanoribbon. J. Am. Chem. Soc. 2020, 142 (15), 7066-7074 https://doi.org/10.1021/jacs.0c00646.

(8) (a) Nuckolls, C.; Katz, T. J.; Castellanos, L. Aggregation of Conjugated Helical Molecules. J. Am. Chem. Soc. 1996, 118 (15), 3767-3768. https://doi.org/10.1021/ja960145z; (b) Hatakeyama, T.; Hashimoto, S.; Oba, T.; Nakamura, M. Azaboradibenzo[6]Helicene: Carrier Inversion Induced by Helical Homochirality. J. Am. Chem. Soc. 2012, 134 (48), 19600-19603. https://doi.org/10.1021/ja310372f; (c) Yang, Y.; Rice, B. Shi, X.; Brandt, J. R.; Correa da Costa, R.; Hedley, G. J.; Smilgies, D.M.; Frost, J. M.; Samuel, I. D. W.; Otero-de-la-Roza, A.; Johnson, E. R.; Jelfs, K. E.; Nelson, J.; Campbell, A. J.; Fuchter, M. J. Emergent Properties of an Organic Semiconductor Driven by Its Molecular Chirality. ACS Nano 2017, $11 \quad$ (8), 8329-8338. https://doi.org/10.1021/acsnano.7b03540; (d) Shcherbina, M. A.; Zeng X.; Tadjiev, T.; Ungar, G.; Eichhorn, S. H.; Phillips, K. E. S.; Katz, T. J. Hollow Six-Stranded Helical Columns of a Helicene. Angew. Chem. Int. Ed. 2009, 48 (42), 7837-7840. https://doi.org/10.1002/anie.200903658.

(9) (a) Kiel, G. R.; Patel, S. C.; Smith, P. W.; Levine, D. S.; Tilley, T. D. Expanded Helicenes: A General Synthetic Strategy and Remarkable Supramolecular and Solid-State Behavior. J. Am. Chem. Soc. 2017, 139 (51), 18456-18459. https://doi.org/10.1021/jacs.7b10902; (b) Nakakuki, Y.; Hirose, T.; Matsuda, K. Synthesis of a Helical Analogue of Kekulene: A Flexible $\pi$-Expanded Helicene with Large Helical Diameter Acting as a Soft Molecular Spring. J. Am. Chem. Soc. 2018, 140 (45), 1546115469. https://doi.org/10.1021/iacs.8b09825; (c) Kiel, G. R.; Bay, K. L.; Samkian, A. E.; Schuster, N. J.; Lin, J. B.; Handford, R. C.; Nuckolls, C.; Houk, K. N.; Tilley, T. D. Expanded Helicenes as Synthons for Chiral Macrocyclic Nanocarbons. J. Am. Chem. Soc. 2020, 142 (25), 11084 11091. https://doi.org/10.1021/jacs.0c03177; (d) Fujise, K.; Tsurumaki, E.; Fukuhara, G.; Hara, N.; Imai, Y.; Toyota, S. Multiply Fused Anthracenes as Helical Polycyclic Aromatic Hydrocarbon Motif for Chiroptical Performance Enhancement. Chem. - Asian J. 2020, 15 (16), 2456-2461. https://doi.org/10.1002/asia.202000394.

(10) (a) Jones, C. G.; Martynowycz, M. W.; Hattne, J.; Fulton, T. J.; Stoltz, B. M.; Rodriguez, J. A.; Nelson, H. M.; Gonen, T. The CryoEM Method MicroED as a Powerful Tool for Small Molecule Structure Determination. ACS Cent. Sci. 2018, 4 (11), 1587-1592. https://doi.org/10.1021/acscentsci.8b00760; (b) Jones, C. G.; Asay, M.; Kim, L. J.; Kleinsasser, J. F.; Saha, A.; Fulton, T. J.; Berkley, K. R.; Cascio, D.; Malyutin, A. G.; Conley, M. P.; Stoltz, B. M.; Lavallo, V.; Rodríguez, J. A.; Nelson, H. M. Characterization of Reactive Organometallic Species via MicroED. ACS Cent. Sci. 2019, 5 (9), 15071513. https://doi.org/10.1021/acscentsci.9b00403; (c) Nannenga, B. L. MicroED Methodology and Development. Struct. Dyn. 2020, 7 (1), 014304. https://doi.org/10.1063/1.5128226; (d) Levine, A. M.; Bu, G.; Biswas, S.; Tsai, E. H. R.; Braunschweig, A. B.; Nannenga, B. L. Crystal Structure and Orientation of Organic Semiconductor Thin Films by Microcrystal Electron Diffraction and Grazing-Incidence Wide-Angle XRay Scattering. Chem. Commun. 2020, 56 (30), 4204-4207. https://doi.org/10.1039/D0CC00119H.

(11) Chen, Z.; Lohr, A.; Saha-Möller, C. R.; Würthner, F. Self-Assembled חStacks of Functional Dyes in Solution: Structural and Thermodynamic Features. Chem. Soc. Rev. 2009, 38 (2), 564-584. https://doi.org/10.1039/B809359H.

(12) (a) Liberko, C. A.; Miller, L. L.; Katz, T. J.; Liu, L. The Electronic Structure of Helicene-Bisquinone Anion Radicals. J. Am. Chem. Soc 1993, 115 (6), 2478-2482. https://doi.org/10.1021/ja00059a050; (b) Schweinfurth, D.; Zalibera, M.; Kathan, M.; Shen, C.; Mazzolini, M.; Trapp, N.; Crassous, J.; Gescheidt, G.; Diederich, F. Helicene Quinones: Redox-Triggered Chiroptical Switching and Chiral Recognition of the Semiquinone Radical Anion Lithium Salt by Electron Nuclear Double Resonance Spectroscopy. J. Am. Chem.
Soc. 2014, $136 \quad$ (37), 13045-13052 https://doi.org/10.1021/ja5069323; (c) Schweinfurth, D.; Mazzolini, M.; Neshchadin, D.; Hoyer, C.; Geier, R.; Gatterer, K.; Trapp, N.; Gescheidt, G.; Diederich, F. Electronic Structures and Chiroptical Properties of Post-Functionalized Helicene Quinones. Chem. - Eur. $\begin{array}{llll}J . & 2016, & 22 & \text { (21), }\end{array}$ https://doi.org/10.1002/chem.201600611

(13) (a) Pascal, R. A.; McMillan, W. D.; Van Engen, D.; Eason, R. G. Synthesis and Structure of Longitudinally Twisted Polycyclic Aromatic Hydrocarbons. J. Am. Chem. Soc. 1987, 109 (15), 4660 4665. https://doi.org/10.1021/ja00249a032; (b) Wang, Z.; Tomović, Ž.; Kastler, M.; Pretsch, R.; Negri, F.; Enkelmann, V.; Müllen, K. Graphitic Molecules with Partial "Zig/Zag" Periphery. J. Am. Chem. $\begin{array}{llll}\text { Soc. 2004, } & 126 & \text { (25), } & \text { 7794-7795 }\end{array}$ https://doi.org/10.1021/ja048580d; (c) Wasserfallen, D.; Kastler, M.; Pisula, W.; Hofer, W. A.; Fogel, Y.; Wang, Z.; Müllen, K. Suppressing Aggregation in a Large Polycyclic Aromatic Hydrocarbon. J. Am. Chem. Soc. 2006, 128 (4), 1334-1339. https://doi.org/10.1021/ja056782j.

(14) Wang, Z.; Lu, P.; Chen, S.; Gao, Z.; Shen, F.; Zhang, W.; Xu, Y.; Kwok, H. S.; Ma, Y. Phenanthro[9,10-d]Imidazole as a New Building Block for Blue Light Emitting Materials. J. Mater. Chem. 2011, 21 (14), 5451-5456. https://doi.org/10.1039/C1JM10321K.

(15) (a) Fogel, Y.; Kastler, M.; Wang, Z.; Andrienko, D.; Bodwell, G. J.; Müllen, K. Electron-Deficient N-Heteroaromatic Linkers for the Elaboration of Large, Soluble Polycyclic Aromatic Hydrocarbons and Their Use in the Synthesis of Some Very Large Transition Metal Complexes. J. Am. Chem. Soc. 2007, 129 (38), 11743-11749. https://doi.org/10.1021/ja072521t; (b) Bunz, U. H. F.; Engelhart, J. U.; Lindner, B. D.; Schaffroth, M. Large N-Heteroacenes: New Tricks for Very Old Dogs? Angew. Chem. Int. Ed. 2013, 52 (14), 3810-3821. https://doi.org/10.1002/anie.201209479; (c) MateoAlonso, A. Pyrene-Fused Pyrazaacenes: From Small Molecules to Nanoribbons. Chem. Soc. Rev. 2014, 43, 6311-6324. http://dx.doi.org/10.1039/c4cs00119b.

(16) (a) Sakai, H.; Shinto, S.; Araki, Y.; Wada, T.; Sakanoue, T.; Takenobu, T.; Hasobe, T. Formation of One-Dimensional Helical Columns and Excimerlike Excited States by Racemic QuinoxalineFused [7]Carbohelicenes in the Crystal. Chem. - Eur. J. 2014, 20 (32), 10099-10109. https://doi.org/10.1002/chem.201402426; (b) Sun, Y.; Li, X.; Sun, C.; Shen, H.; Hou, X.; Lin, D.; Zhang, H. -L.; Di, C.; Zhu, D.; Shao, X. Trichalcogenasumanene Ortho-Quinones: Synthesis, Properties, and Transformation into Various Heteropolycycles. Angew. Chem. Int. Ed. 2017, 56 (43), 1347013474. https://doi.org/10.1002/anie.201707397.

(17) (a) Boden, B. N.; Jardine, K. J.; Leung, A. C. W.; MacLachlan, M. J. Tetraalkoxyphenanthrene: A New Precursor for Luminescent Conjugated Polymers. Org. Lett. 2006, 8 (9), 1855-1858. https://doi.org/10.1021/ol060433s; (b) Rieger, R.; Kastler, M.; Enkelmann, V.; Müllen, K. Entry to Coronene Chemistry-Making Large Electron Donors and Acceptors. Chem. - Eur. J. 2008, 14 (21), 6322-6325. https://doi.org/10.1002/chem.200800832.

(18) Solà, M. Forty Years of Clar's Aromatic m-Sextet Rule. Front. Chem. 2013, 1. https://doi.org/10.3389/fchem.2013.00022.

(19) Moriconi, E. J.; Rakoczy, B.; O'Connor, W. F. Oxidation-Reduction Potentials and Absorption Spectra of Polycyclic Aromatic Quinones1. J. Org. Chem. 1962, 27 (8), 2772-2776. https://doi.org/10.1021/j001055a016.

(20) Togashi, D. M.; Nicodem, D. E. Photophysical Studies of 9,10Phenanthrenequinones. Spectrochim. Acta. A. Mol. Biomol. Spectrosc. 2004, 60 (13), 3205-3212. https://doi.org/10.1016/i.saa.2004.03.003.

(21) CV data was also acquired in THF solution (Figure S21), which is a better solvent for probing reduction events. For example, 2-mon exhibits two reversible waves in THF versus only one in $\mathrm{CH}_{2} \mathrm{Cl}_{2}$.

(22) Due to the dynamic nature of the self-assembling system, the measurements do not yield accurate information on specific aggregate size, but rather function as a tool for qualitative comparisons. See also: Wang, W.; Han, J. J.; Wang, L.-Q.; Li, L.-S.; 
Shaw, W. J.; Li, A. D. Q.; Dynamic $\pi-\pi$ Stacked Molecular Assemblies Emit from Green to Red Colors. Nano Lett. 2003, 3 (4), 455-458. https://doi.org/10.1021/nl025976j.

(23) (a) Shetty, A. S.; Zhang, J.; Moore, J. S. Aromatic m-Stacking in Solution as Revealed through the Aggregation of Phenylacetylene Macrocycles. J. Am. Chem. Soc. 1996, 118 (5), 1019-1027. https://doi.org/10.1021/ja9528893; (b) Horman, I.; Dreux, B. Estimation of Dimerisation Constants from Complexatin-Induced Displacements of $1 \mathrm{H}$-NMR Chemical Shifts: Dimerisation of Caffeine. Helv. Chim. Acta 1984, 67 (3), 754-764. https://doi.org/10.1002/hlca.19840670316.

(24) In general, crystals were $10-50 \mu \mathrm{m}$ by $1-2 \mu \mathrm{m}$ needles or rods.

(25) Pitches were calculated by fitting a helical curve to the 11 centroids of the inner rings, using the HELFIT program developed by Enkhbayar and coworkers (see SI for details): Enkhbayar, P.; Damdinsuren, S.; Osaki, M.; Matsushima, N. HELFIT: Helix Fitting by a Total Least Squares Method. Comput. Biol. Chem. 2008, 32 (4), 307-310. https://doi.org/10.1016/i.compbiolchem.2008.03.012. 
Entry for the Table of Contents
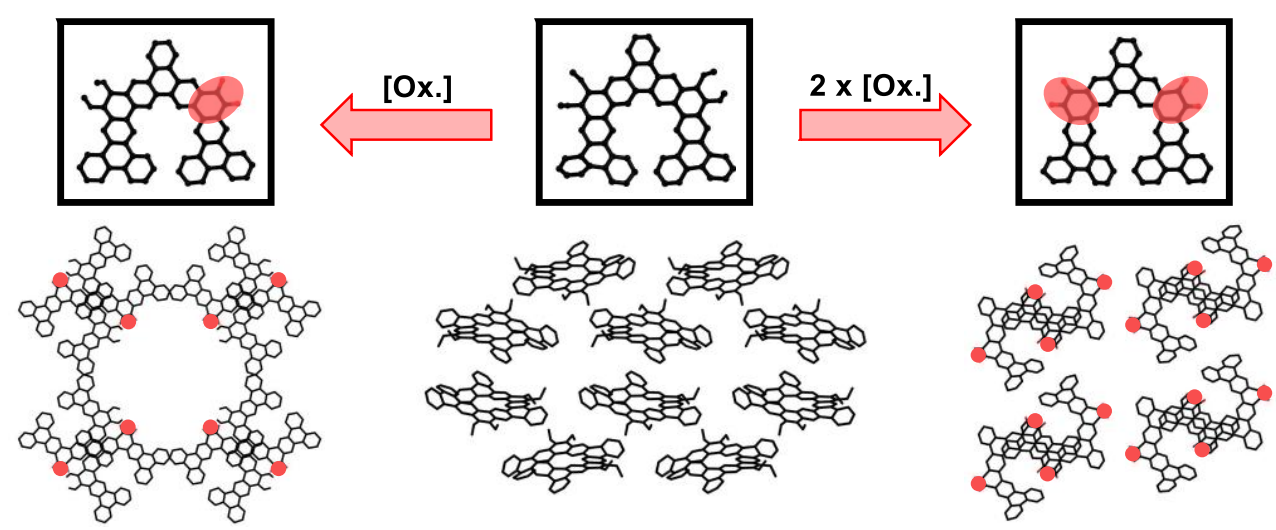

- Oxidation state dictates crystal packing

- MicroED structural elucidation 
Supporting Information for:

\section{MicroED Elucidation of Diverse Solid-State Packing in a Family of Electron-Deficient Expanded Helicenes}

Adrian E. Samkian, ${ }^{[a]}$ Gavin R. Kiel, ${ }^{[a]}$ Christopher G. Jones, ${ }^{[b]}$ Harrison M. Bergman, ${ }^{[a]}$ Julia Oktawiec, ${ }^{[a]}$ Hosea M. Nelson, ${ }^{*[b]}$ and T. Don Tilley ${ }^{*[a]}$

[a] Department of Chemistry, University of California, Berkeley, Berkeley, California, 94720, United States

[b] Department of Chemistry and Biochemistry, University of California, Los Angeles, Los Angeles, California 90095, United States

\section{Table of Contents:}

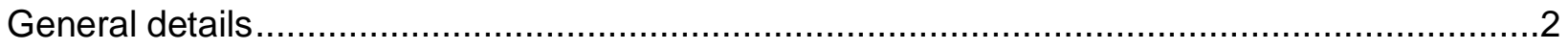

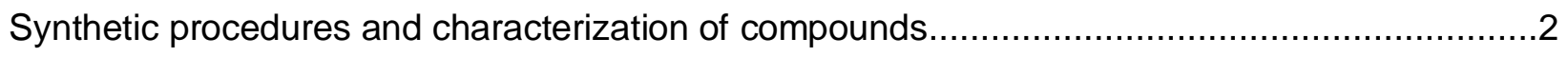

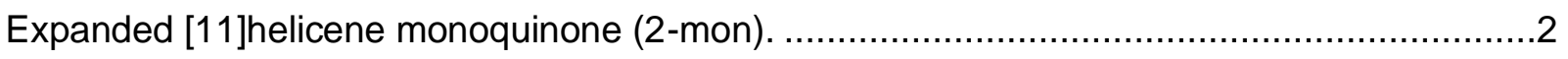

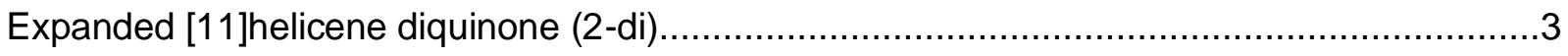

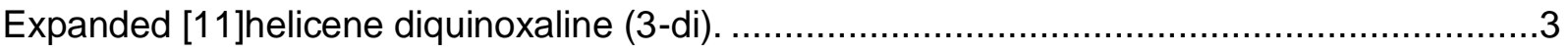

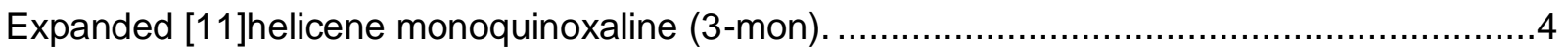

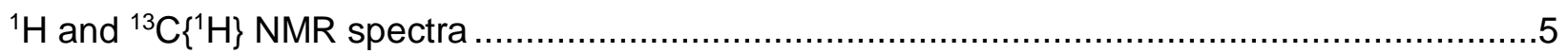

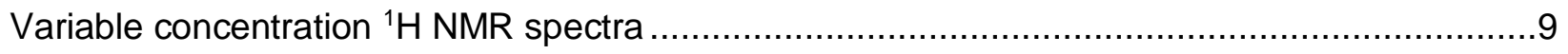

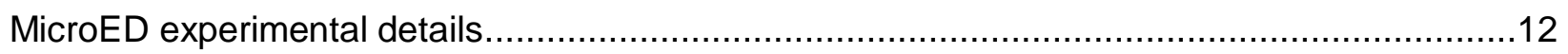

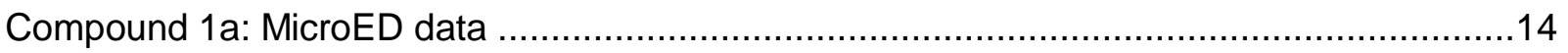

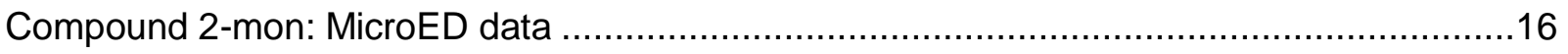

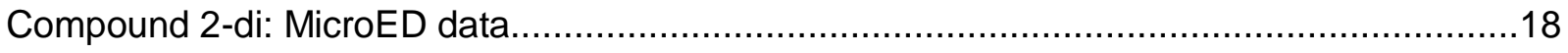

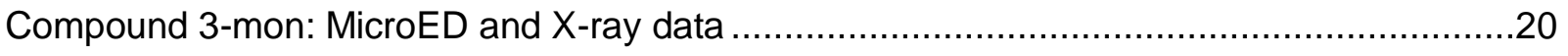

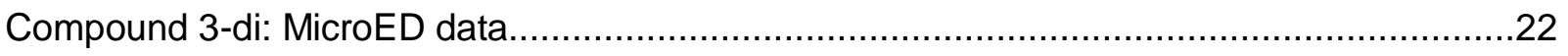

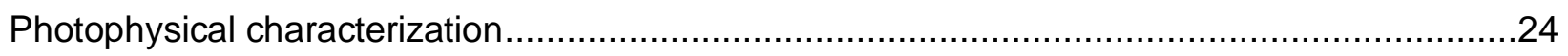

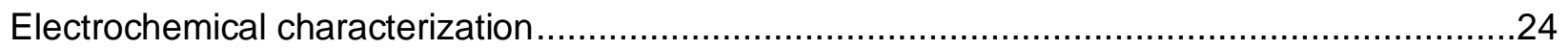

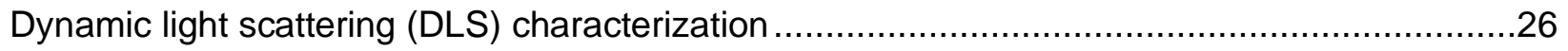

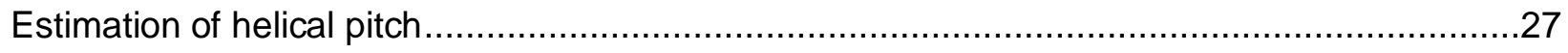

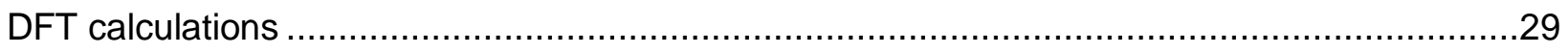

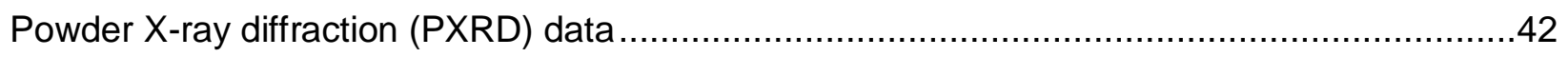

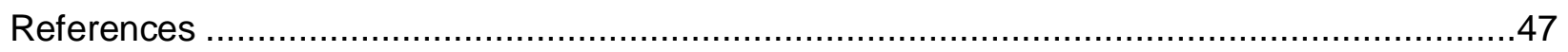




\section{General details}

Commercial cerium ammonium nitrate (CAN) from Sigma-Aldrich was purified according to a literature procedure, ${ }^{1}$ o-phenylenediamine was purified by sublimation under vacuum $(15 \mathrm{mmHg}$, $\left.50{ }^{\circ} \mathrm{C}\right)$. Compound 1a was prepared according to the literature. ${ }^{2}$ All other reagents and solvents were purchased from commercial suppliers and used as received. Mass spectrometry was performed by the QB3/Chemistry Mass Spectrometry Facility at the University of California, Berkeley. Preparatory thin layer chromatography was carried out using Analtech 1000-micron preparatory plates. Unless otherwise noted, NMR spectra were acquired at ambient temperature $\left(\sim 22^{\circ} \mathrm{C}\right)$ using Bruker AV-700, AV-600, AV-500, DRX-500, AV-400, and AV-300 spectrometers. Chemical shifts $(\delta)$ are given in ppm and referenced to residual solvent peaks for ${ }^{1} \mathrm{H}$ NMR spectra $(\delta=7.26 \mathrm{ppm}$ for chloroform- $d)$ and for ${ }^{13} \mathrm{C}\left\{{ }^{1} \mathrm{H}\right\}$ NMR spectra $(\delta=77.16 \mathrm{ppm}$ for chloroform- $d)$.

\section{Synthetic procedures and characterization of compounds}

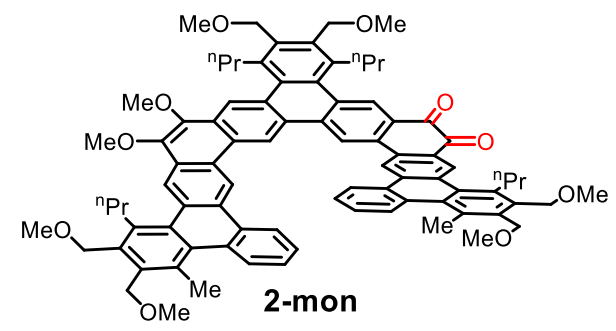

\section{Expanded [11]helicene monoquinone (2-mon).}

A $20 \mathrm{~mL}$ vial was charged with compound $1 \mathrm{a}\left(100.0 \mathrm{mg}, 0.076 \mathrm{mmol}, 1.0\right.$ equiv) and $\mathrm{CH}_{2} \mathrm{Cl}_{2}(2.5$ $\mathrm{mL}$ ) to form a homogeneous solution, then the solution was diluted with MeCN (1.3 mL) (to avoid solubility issues, the order of addition is important). To the stirred solution was added a solution of cerium ammonium nitrate (251 $\mathrm{mg}, 0.458 \mathrm{mmol}, 6.0$ equiv) in water ( $4.6 \mathrm{~mL}$ ). The biphasic mixture was stirred vigorously (i.e., at a rate such that the phases become indistinguishable) for $3 \mathrm{~h}$, then diluted with water $(10 \mathrm{~mL})$ and $\mathrm{CH}_{2} \mathrm{Cl}_{2}(50-60 \mathrm{~mL}$, or until the organic layer became transparent). The layers were separated, and the organic layer was dried with $\mathrm{MgSO}_{4}$, filtered, and the filtrate was concentrated by rotary evaporation. The residue was subjected to preparatory thin layer chromatography $\left(15: 1 \mathrm{CH}_{2} \mathrm{Cl}_{2}: \mathrm{MeCN}\right)$ to afford 2-mon $(81 \mathrm{mg}, 84 \%)$ as a red solid. ${ }^{1} \mathrm{H}$ NMR (chloroform- $d, 600 \mathrm{MHz}$ ): $\delta=10.06(\mathrm{~s}, 1 \mathrm{H}), 10.04(\mathrm{~s}, 1 \mathrm{H}), 9.54(\mathrm{~s}, 1 \mathrm{H}), 9.47(\mathrm{~s}, 1 \mathrm{H}), 9.08$ (s, 1H), $9.06(\mathrm{~s}, 1 \mathrm{H}), 9.03(\mathrm{~s}, 1 \mathrm{H}), 9.00(\mathrm{~s}, 1 \mathrm{H}), 8.96(\mathrm{~d}, \mathrm{~J}=7.9 \mathrm{~Hz}, 1 \mathrm{H}), 8.91(\mathrm{~d}, \mathrm{~J}=7.9 \mathrm{~Hz}, 1 \mathrm{H})$, $8.25(\mathrm{dd}, \mathrm{J}=12.9,8.0 \mathrm{~Hz}, 2 \mathrm{H}), 7.64-7.40(\mathrm{~m}, 4 \mathrm{H}), 4.83(\mathrm{~s}, 2 \mathrm{H}), 4.82-4.78(\mathrm{~m}, 6 \mathrm{H}), 4.77(\mathrm{~s}$, $2 \mathrm{H}), 4.73(\mathrm{~s}, 2 \mathrm{H}), 4.21(\mathrm{~s}, 3 \mathrm{H}), 4.19(\mathrm{~s}, 3 \mathrm{H}), 3.62(\mathrm{~s}, 3 \mathrm{H}), 3.61(\mathrm{~s}, 3 \mathrm{H}), 3.61(\mathrm{~s}, 6 \mathrm{H}), 3.57(\mathrm{~s}, 3 \mathrm{H})$, $3.57(\mathrm{~s}, 3 \mathrm{H}), 3.52-3.41(\mathrm{~m}, 4 \mathrm{H}), 3.35-3.24(\mathrm{~m}, 4 \mathrm{H}), 2.98(\mathrm{~s}, 3 \mathrm{H}), 2.97(\mathrm{~s}, 3 \mathrm{H}), 2.17-2.08(\mathrm{~m}$, 4H), $2.01-1.91(\mathrm{~m}, 4 \mathrm{H}), 1.19-1.12(\mathrm{~m}, 6 \mathrm{H}), 1.08(\mathrm{t}, \mathrm{J}=7.3 \mathrm{~Hz}, 3 \mathrm{H}), 1.03$ (t, J = 7.3 Hz, 3H); ${ }^{13} \mathrm{C}\left\{{ }^{1} \mathrm{H}\right\}$ NMR (chloroform- $\left.d, 151 \mathrm{MHz}\right): \delta=180.61,180.48,145.02,143.81,137.49,137.19$, $137.17,137.11,136.83,136.83,136.75,136.62$, 136.29, 136.13, 135.61, 134.93, 134.47, 134.41, 134.38, 133.51, 133.26, 132.97, 132.93, 132.82, 132.48, 132.42, 132.40, 132.32, 132.30, 132.00, 131.87, 131.73, 131.73, 131.57, 130.91, 130.24, 129.80, 129.79, 129.73, 129.62, 129.34, 129.05, 128.39, 128.21, 127.98, 127.93, 127.87, 127.44, 127.11, 126.42, 123.64, 123.08, 122.79, 122.78, 119.31, 119.05, 118.56, 117.37, 69.34, 69.29, 69.27, 69.07, 69.02, 68.98, 61.08, 61.04, 59.02, $58.99,58.97,58.94,58.86,58.82$, 35.28, 35.19, 35.05, 34.99, 26.02, 25.97, 25.83, 25.77, 20.69, 20.68, 14.69, 14.68, 14.59, 14.58; HRMS-ESI (m/z): $[\mathrm{M}+\mathrm{H}]^{+}$calcd. for $\mathrm{C}_{86} \mathrm{H}_{87} \mathrm{O}_{10}, 1279.6294$; found, 1279.6288. 


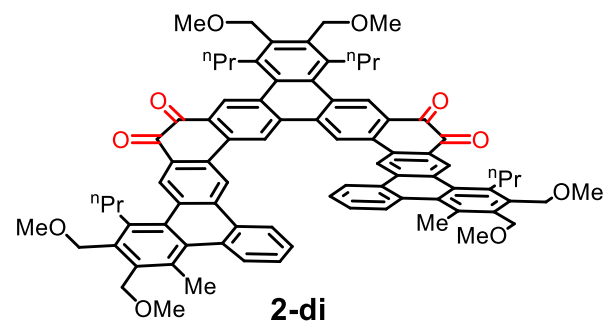

\section{Expanded [11]helicene diquinone (2-di).}

A $20 \mathrm{~mL}$ screw-cap vial was charged with compound $1 \mathrm{a}$ (100 mg, $0.076 \mathrm{mmol}, 1.0$ equiv), $\mathrm{CH}_{2} \mathrm{Cl}_{2}$ $(5.1 \mathrm{~mL})$, and $\mathrm{MeCN}(2.5 \mathrm{~mL})$ (to avoid solubility issues, the order of addition is important). To the stirred solution was added a solution of cerium ammonium nitrate $(628 \mathrm{mg}, 1.15 \mathrm{mmol}, 15$ equiv) in water $(8.9 \mathrm{~mL})$. The biphasic mixture was stirred vigorously (i.e., at a rate such that the phases become indistinguishable) for $5 \mathrm{~h}$, then diluted with $\mathrm{CH}_{2} \mathrm{Cl}_{2}(30 \mathrm{~mL})$. The layers were separated, and the organic layer was dried with $\mathrm{MgSO}_{4}$, filtered, and the filtrate was concentrated by rotary evaporation. The residue was subjected to preparatory thin layer chromatography (15:1 $\mathrm{CH}_{2} \mathrm{Cl}_{2}: \mathrm{MeCN}$ ) to afford 2-di (72 mg, 76\%) as a red solid. ${ }^{1} \mathrm{H}$ NMR (chloroform-d, $500 \mathrm{MHz}$, [c] = $80 \mathrm{mM}$ ): $\delta=9.39(\mathrm{~s}, 2 \mathrm{H}), 9.14(\mathrm{~s}, 2 \mathrm{H}), 9.02(\mathrm{~s}, 2 \mathrm{H}), 8.78(\mathrm{~s}, 2 \mathrm{H}), 8.64(\mathrm{~s}, 2 \mathrm{H}), 8.22(\mathrm{~d}, J=7.8 \mathrm{~Hz}$, $2 \mathrm{H}), 7.58(\mathrm{q}, J=11.1,9.0 \mathrm{~Hz}, 4 \mathrm{H}), 4.74(\mathrm{~s}, 4 \mathrm{H}), 4.62(\mathrm{~s}, 4 \mathrm{H}), 4.51(\mathrm{~s}, 4 \mathrm{H}), 3.58(\mathrm{~s}, 6 \mathrm{H}), 3.51(\mathrm{~s}$, $6 \mathrm{H}), 3.47(\mathrm{~s}, 6 \mathrm{H}), 3.08-2.97(\mathrm{~m}, 4 \mathrm{H}), 2.92(\mathrm{~s}, 6 \mathrm{H}), 2.89-2.71(\mathrm{~m}, 4 \mathrm{H}), 1.90-1.70(\mathrm{~m}, 8 \mathrm{H}), 0.85$ $\left(\mathrm{s}, 12 \mathrm{H}\right.$ ); ${ }^{13} \mathrm{C}\left\{{ }^{1} \mathrm{H}\right\}$ NMR (chloroform-d, $126 \mathrm{MHz}$, [c] = $80 \mathrm{mM}$ ): $\delta=180.74,180.24,138.02,137.27$, 136.99, 136.89, 136.31, 135.48, 134.96, 134.40, 133.00, 132.64, 132.49, 132.43, 132.25. 132.19, $131.81,131.80,131.68,131.50,130.07,130.06,129.56,128.79,128.21,127.93,124.39,119.72$, $119.44,69.30,68.90,68.57,58.96,58.85,58.72,35.06,34.95,25.64,25.28,20.66,14.37,14.15$; HRMS-ESI (m/z): $[\mathrm{M}+\mathrm{H}]^{+}$calcd. for $\mathrm{C}_{84} \mathrm{H}_{81} \mathrm{O}_{10}, 1249.5824$; found, 1249.5848 .

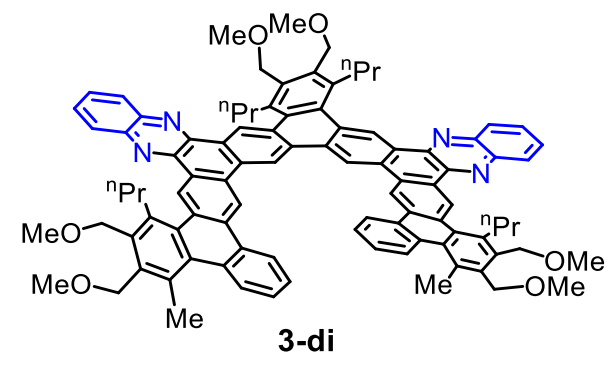

Expanded [11]helicene diquinoxaline (3-di).

A $20 \mathrm{~mL}$ screw-cap vial was charged with compound 2-di (72 mg, $0.058 \mathrm{mmol}, 1.0$ equiv), ophenylenediamine (125 mg, $1.15 \mathrm{mmol}, 20.0$ equiv), and 1,2-dichloroethane (4 mL). The vial was sealed, then the stirred mixture was heated at $80^{\circ} \mathrm{C}$ for $16 \mathrm{~h}$, brought to RT, and concentrated under reduced pressure. To remove excess o-phenylenediamine, the residue was dissolved in $\mathrm{CH}_{2} \mathrm{Cl}_{2}(3 \mathrm{~mL})$, then the solution was diluted with $\mathrm{MeCN}(3 \mathrm{~mL})$ and concentrated to $\sim 2 \mathrm{~mL}$ via rotary evaporation. The resulting precipitate was collected by filtration, washed with $\mathrm{MeCN}(2 \mathrm{~mL})$, and recrystallized from toluene $(7 \mathrm{~mL})$ at $-25^{\circ} \mathrm{C}$ to afford 3-di $(70 \mathrm{mg}, 89 \%)$ as a yellow needles. * ${ }^{1} \mathrm{H}$ NMR (chloroform- $\left.d, 500 \mathrm{MHz},[\mathrm{c}]=24 \mathrm{mM}\right): \delta=10.28(\mathrm{~s}, 4 \mathrm{H}), 10.12(\mathrm{~s}, 2 \mathrm{H}), 10.04(\mathrm{~s}, 2 \mathrm{H})$, $9.06(\mathrm{~d}, J=7.7 \mathrm{~Hz}, 2 \mathrm{H}), 8.32(\mathrm{~d}, J=9.7 \mathrm{~Hz}, 6 \mathrm{H}), 7.86(\mathrm{dd}, J=6.4,3.3 \mathrm{~Hz}, 4 \mathrm{H}), 7.57$ (dt, $J=20.1$, $7.2 \mathrm{~Hz}, 4 \mathrm{H}), 4.91(\mathrm{~s}, 4 \mathrm{H}), 4.86(\mathrm{~d}, J=5.5 \mathrm{~Hz}, 8 \mathrm{H}), 3.65(\mathrm{~s}, 6 \mathrm{H}), 3.63(\mathrm{~s}, 6 \mathrm{H}), 3.60(\mathrm{~s}, 6 \mathrm{H}), 3.49(\mathrm{~s}$, $8 \mathrm{H}), 3.02(\mathrm{~s}, 6 \mathrm{H}), 2.51(\mathrm{~s}, 8 \mathrm{H}), 1.28-1.19(\mathrm{~m}, 12 \mathrm{H}) ;{ }^{13} \mathrm{C}\left\{{ }^{1} \mathrm{H}\right\} \mathrm{NMR}$ (chloroform-d, $151 \mathrm{MHz},[\mathrm{c}]=$ $24 \mathrm{mM}$ ): $\delta=143.01,142.97,142.17,142.13,136.91,136.69,136.43,136.21,135.05,134.32$, 133.89, 133.10, 132.70, 132.65, 132.00, 131.81, 131.54, 130.91, 130.75, 129.80, 129.74, 129.49, $129.47,129.45,129.45,129.29,129.18,128.35,128.02,127.03,126.83,126.33,122.95,117.72$, 
$117.13,69.47,69.18,69.12,58.82,58.76,58.65,53.55,35.67,25.63,25.34,20.66,15.37,15.32$; $[\mathrm{M}+\mathrm{H}]^{+}$calcd. for $\mathrm{C}_{96} \mathrm{H}_{89} \mathrm{O}_{6} \mathrm{~N}_{4}, 1393.6777$; found, 1393.6803 .

${ }^{*}$ Crystals isolated in this way possess low kinetic solubility, requiring sonication or vigorous stirring to re-dissolve. Solutions of 3-di in $\mathrm{CH}_{2} \mathrm{Cl}_{2}$ can be directly evaporated to yield a yellow amorphous film that is easily re-dissolved.

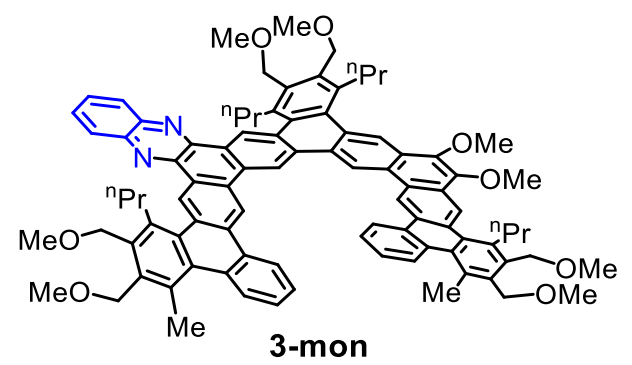

Expanded [11] helicene monoquinoxaline (3-mon).

A $25 \mathrm{~mL}$ Teflon-stoppered flask was loaded with compound 2-mon $(95 \mathrm{mg}, 0.074 \mathrm{mmol}$. 1.0 equiv), o-phenylenediamine (40 mg, $0.37 \mathrm{mmol}, 5$ equiv), and 1,2-dichloroethane $(5 \mathrm{~mL})$. The flask was sealed, then the stirred mixture was heated at $70^{\circ} \mathrm{C}$ for $2 \mathrm{~h}$, brought to RT, and concentrated under reduced pressure. To remove excess $o$-phenylenediamine, the residue was dissolved in $\mathrm{CH}_{2} \mathrm{Cl}_{2}(5 \mathrm{~mL})$, the solution was diluted with $\mathrm{MeCN}(3 \mathrm{~mL})$, and the volume was reduced to $\sim 2 \mathrm{~mL}$ via rotary evaporation. The resulting precipitate was collected by filtration, washed with $\mathrm{MeCN}(3 \mathrm{~mL})$, and recrystallized from toluene at $-25^{\circ} \mathrm{C}$ to afford 3-mon $(67 \mathrm{mg}$, $67 \%$ ) as a yellow microcrystalline solid. ${ }^{~}{ }^{1} \mathrm{H}$ NMR (chloroform-d, $600 \mathrm{MHz}$, [c] = $62 \mathrm{mM}$ ): $\delta=10.14$ (s, 1H), $10.12(\mathrm{~s}, 1 \mathrm{H}), 10.10(\mathrm{~s}, 1 \mathrm{H}), 10.07(\mathrm{~s}, 1 \mathrm{H}), 9.94(\mathrm{~s}, 1 \mathrm{H}), 9.89(\mathrm{~s}, 1 \mathrm{H}), 9.12(\mathrm{~s}, 1 \mathrm{H}), 9.10$ (s, 1H), 9.02 (dd, $J=7.3,2.0 \mathrm{~Hz}, 1 \mathrm{H}$ ), $8.98(\mathrm{~s}, 1 \mathrm{H}), 8.27$ (dd, $J=7.3,2.0 \mathrm{~Hz}, 2 \mathrm{H}), 8.22$ (dd, $J=$ $17.1,7.7 \mathrm{~Hz}, 2 \mathrm{H}), 7.85-7.78(\mathrm{~m}, 2 \mathrm{H}), 7.59-7.53(\mathrm{~m}, 4 \mathrm{H}), 4.88(\mathrm{~s}, 2 \mathrm{H}), 4.85(\mathrm{~s}, 2 \mathrm{H}), 4.84-4.81$ $(\mathrm{m}, 6 \mathrm{H}), 4.79(\mathrm{~s}, 2 \mathrm{H}), 4.24(\mathrm{~s}, 3 \mathrm{H}), 4.23(\mathrm{~s}, 3 \mathrm{H}), 3.63(\mathrm{~s}, 3 \mathrm{H}), 3.61(\mathrm{~s}, 6 \mathrm{H}), 3.60(\mathrm{~s}, 3 \mathrm{H}), 3.60(\mathrm{~s}$, 3H), $3.58-3.51(\mathrm{~m}, 7 \mathrm{H}), 3.41(\mathrm{~s}, 4 \mathrm{H}), 3.02(\mathrm{~s}, 3 \mathrm{H}), 2.99(\mathrm{~s}, 3 \mathrm{H}), 2.40(\mathrm{~d}, J=37.1 \mathrm{~Hz}, 4 \mathrm{H}), 2.10$ (q, $J=7.7 \mathrm{~Hz}, 4 \mathrm{H}), 1.26$ (t, $J=7.3 \mathrm{~Hz}, 3 \mathrm{H}), 1.19(\mathrm{t}, J=7.3 \mathrm{~Hz}, 3 \mathrm{H}), 1.15(\mathrm{t}, J=7.3 \mathrm{~Hz}, 3 \mathrm{H}), 1.11$ $(\mathrm{t}, J=7.3 \mathrm{~Hz}, 3 \mathrm{H}) ;{ }^{13} \mathrm{C}\left\{{ }^{1} \mathrm{H}\right\} \mathrm{NMR}$ (chloroform- $\left.d, 151 \mathrm{MHz},[\mathrm{c}]=62 \mathrm{mM}\right): \delta=144.42,144.21,143.42$, $142.44,136.88,136.58,136.53,136.53,136.49,136.36,136.15,136.15,135.19,134.85,134.56$, 134.47, 134.44, 134.21, 133.70, 133.41, 133.37, 132.84, 132.11, 132.11, 132.10, 131.99, 131.75, $131.70,131.62,131.52,131.19,131.09,130.74,130.35,130.11,129.73,129.73,129.72,129.69$, $129.63,129.63,129.59,129.59,129.49,129.11,128.74,128.68,128.04,128.00,128.00,127.43$, 127.40, 127.31, 127.15, 126.71, 126.38, 123.27, 123.19, 122.83, 122.68, 118.16, 117.98, 117.60, $117.47,69.45,69.39,69.35,69.24,69.23,69.18,61.12,61.09,58.96,58.92,58.90,58.86,58.80$, $58.74,35.68,35.53,35.31,35.25,26.08,25.90,25.83,25.61,20.74,20.72,15.34,15.29,14.71$, 14.70; $[\mathrm{M}+\mathrm{H}]^{+}$calcd. for $\mathrm{C}_{92} \mathrm{H}_{91} \mathrm{O}_{8} \mathrm{~N}_{2}, 1351.6770$; found, 1351.6773 .

${ }^{*}$ Crystals isolated in this way possess low kinetic solubility, requiring sonication or vigorous stirring to re-dissolve. Solutions of 3-mon in $\mathrm{CH}_{2} \mathrm{Cl}_{2}$ can be directly evaporated to yield a yellow amorphous film that is easily re-dissolved. 


\section{${ }^{1} \mathrm{H}$ and ${ }^{13} \mathrm{C}\left\{{ }^{1} \mathrm{H}\right\}$ NMR spectra}

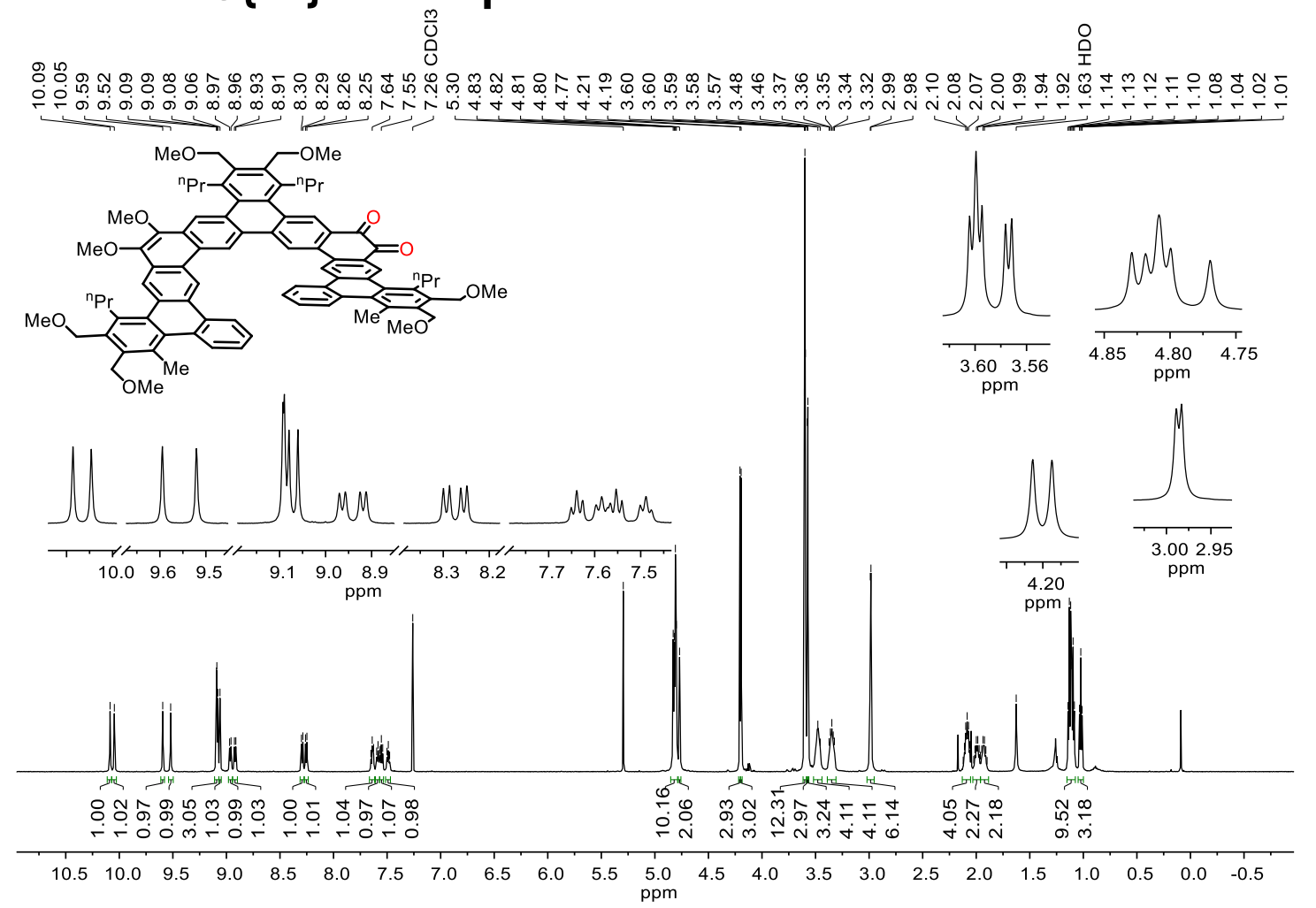

Figure S1. ${ }^{1} \mathrm{H}$ NMR spectrum (600 MHz, chloroform- $d$ ) of 2-mon.

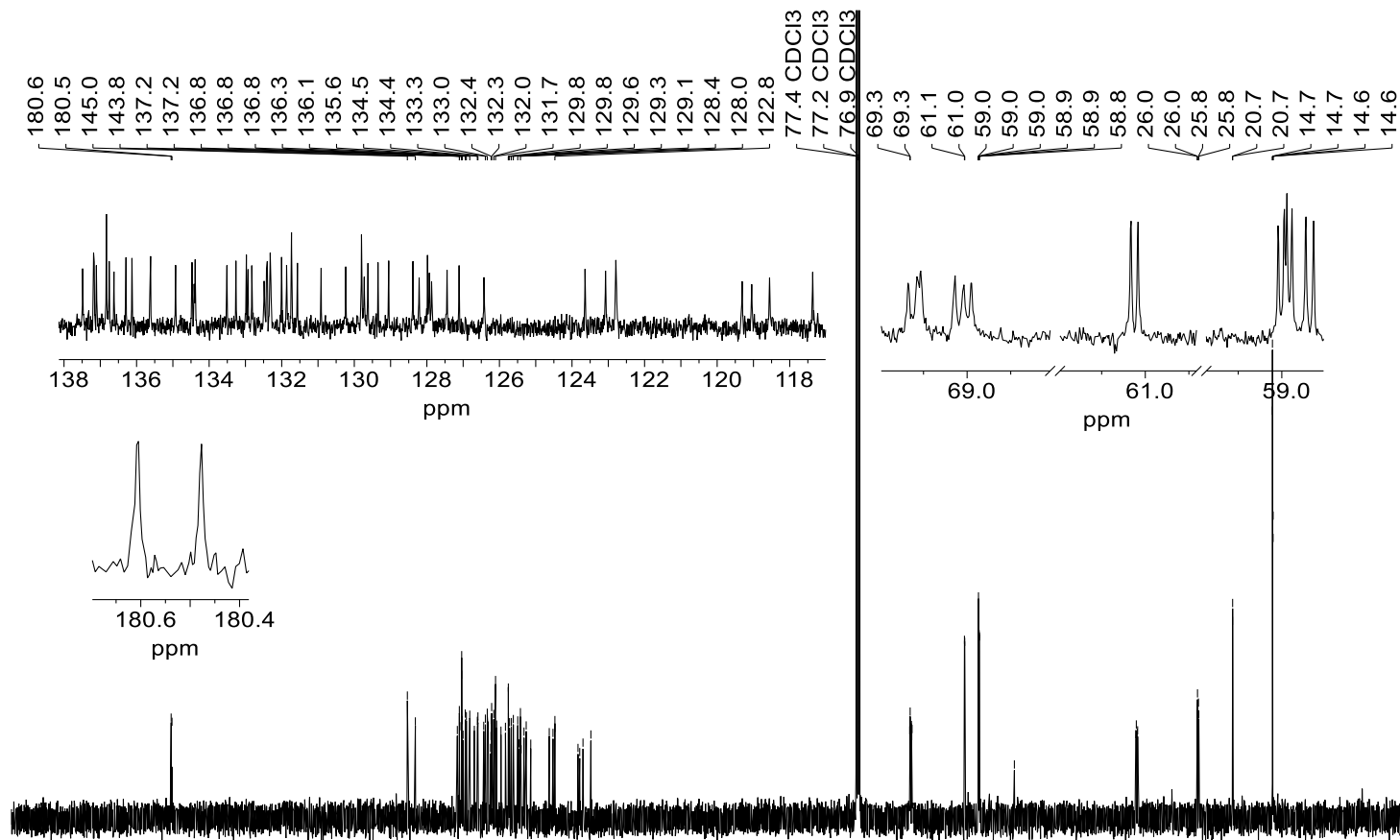

$\begin{array}{lllllllllllllllllllll}200 & 190 & 180 & 170 & 160 & 150 & 140 & 130 & 120 & 110 & \begin{array}{c}100 \\ \mathrm{ppm}\end{array} & 90 & 80 & 70 & 60 & 50 & 40 & 30 & 20 & 10 & 0\end{array}$

Figure S2. ${ }^{13} \mathrm{C}\left\{{ }^{1} \mathrm{H}\right\} \mathrm{NMR}$ spectrum (151 MHz, chloroform- $d$ ) of 2-mon. 


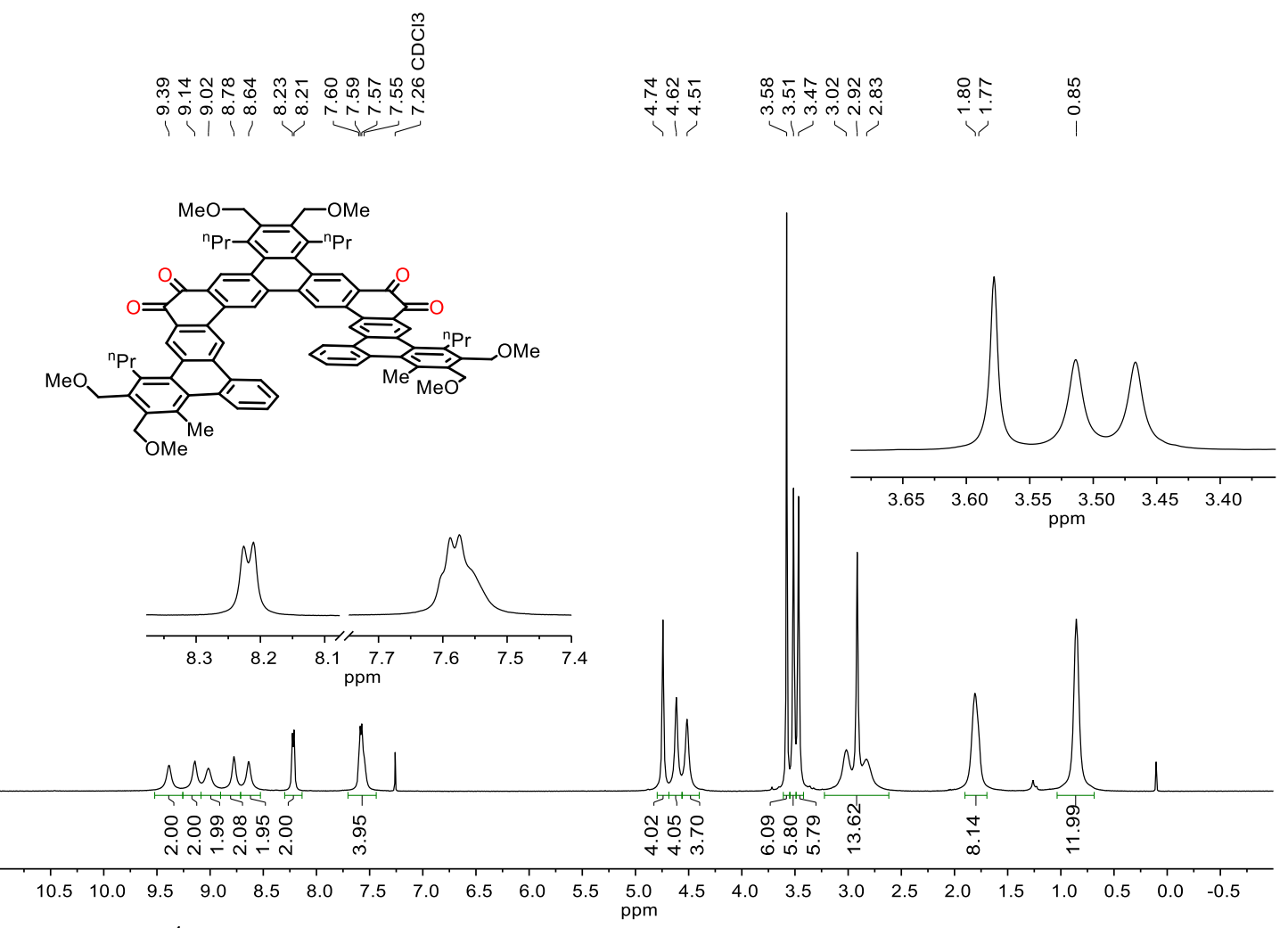

Figure S3. ${ }^{1} \mathrm{H}$ NMR spectrum (500 MHz, chloroform-d, [c] = $80 \mathrm{mM}$ ) of 2-di.

\section{춧숭ำ}

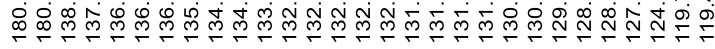
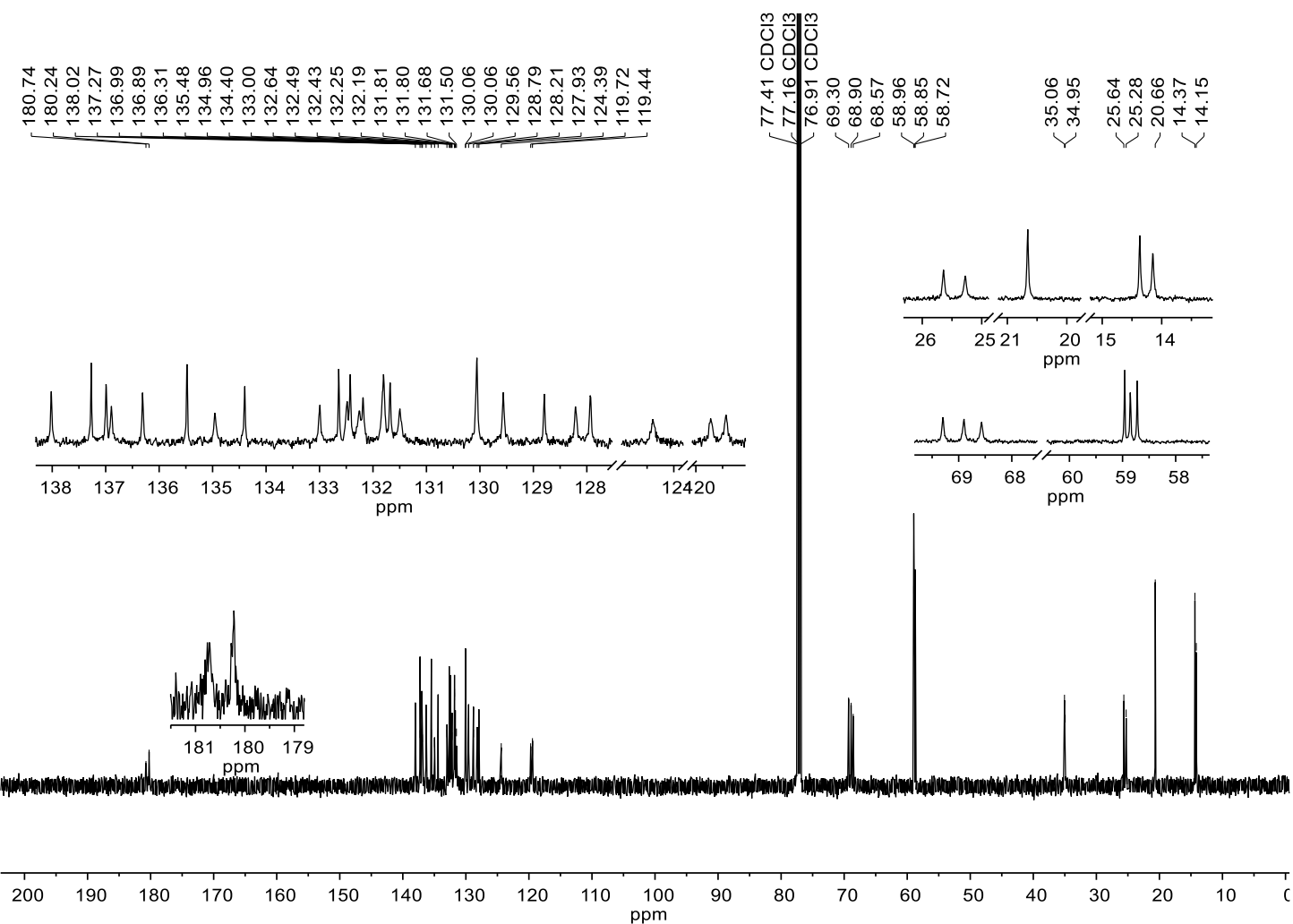

Figure S4. ${ }^{13} \mathrm{C}\left\{{ }^{1} \mathrm{H}\right\}$ NMR spectrum (126 MHz, chloroform- $d$, $[\mathrm{c}]=80 \mathrm{mM}$ ) of 2-di. 


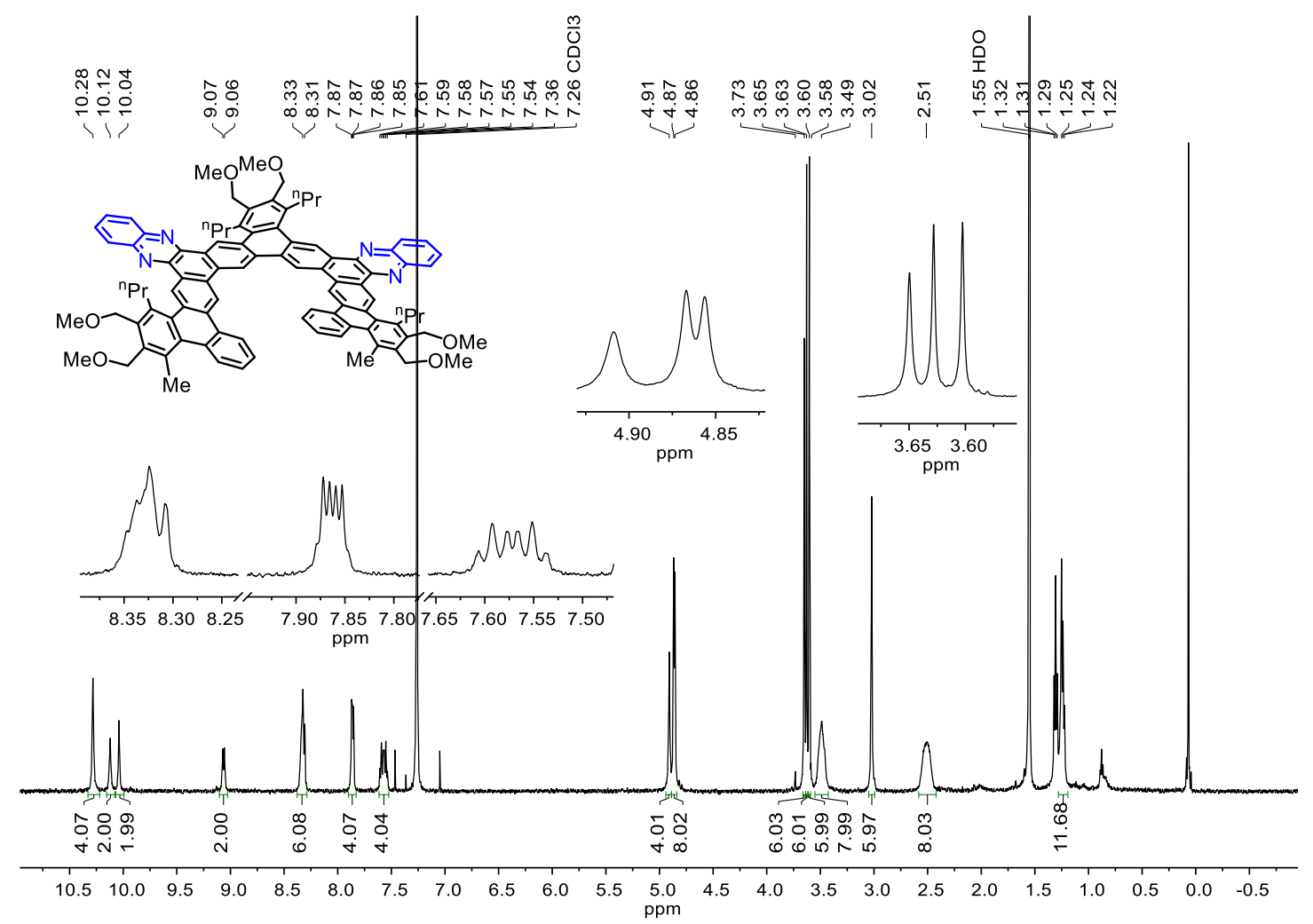

Figure S5. ${ }^{1} \mathrm{H}$ NMR spectrum (500 MHz, chloroform- $d$, [c] = $24 \mathrm{mM}$ ) of 3-di.

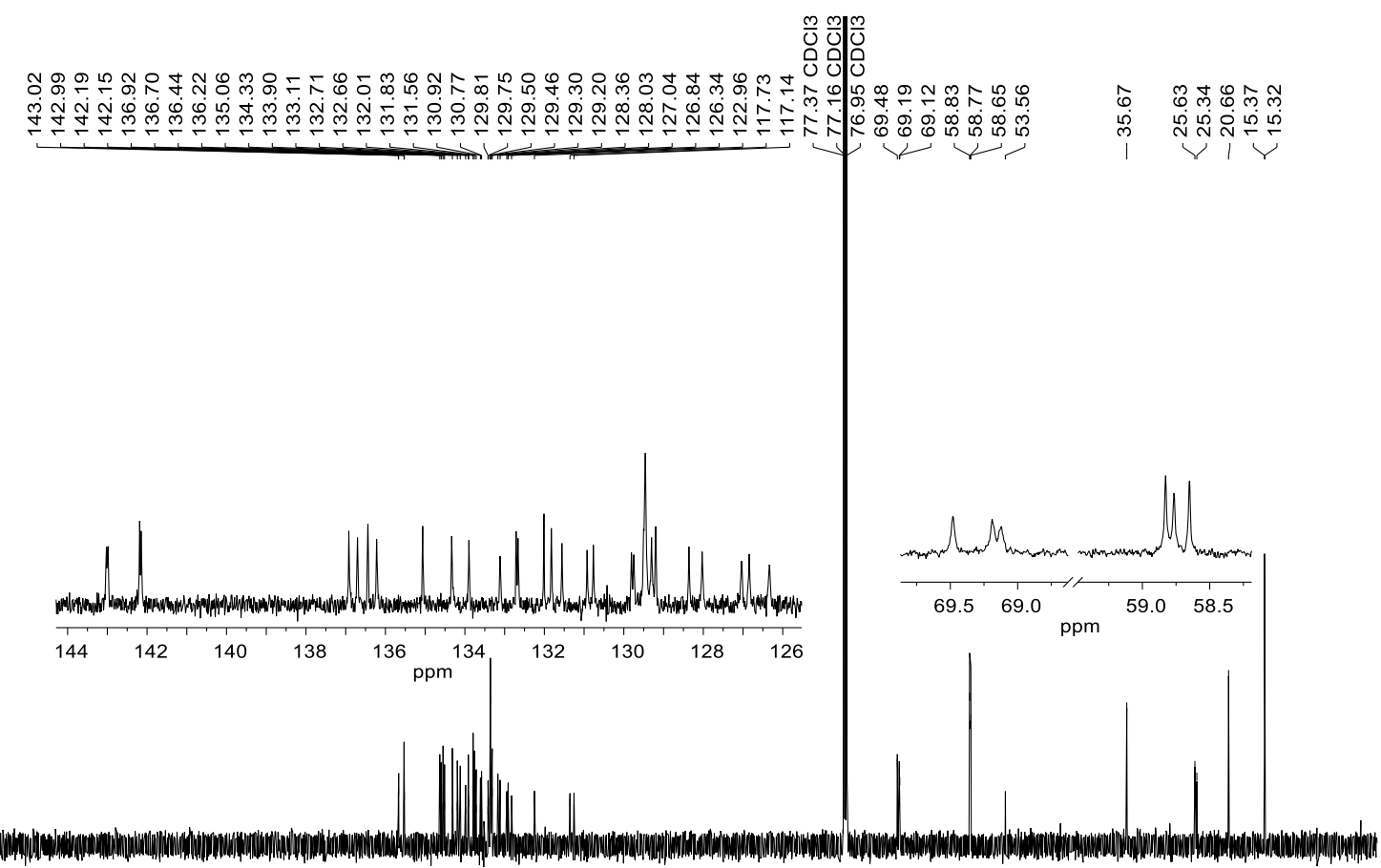

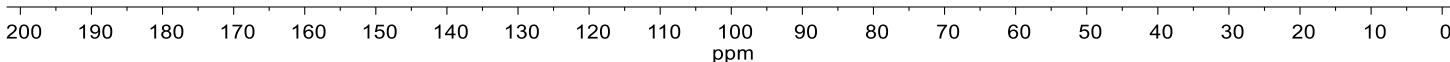

Figure S6. ${ }^{13} \mathrm{C}\left\{{ }^{1} \mathrm{H}\right\}$ NMR spectrum (151 MHz, chloroform- $d$, [c] = $24 \mathrm{mM}$ ) of 3-di. 


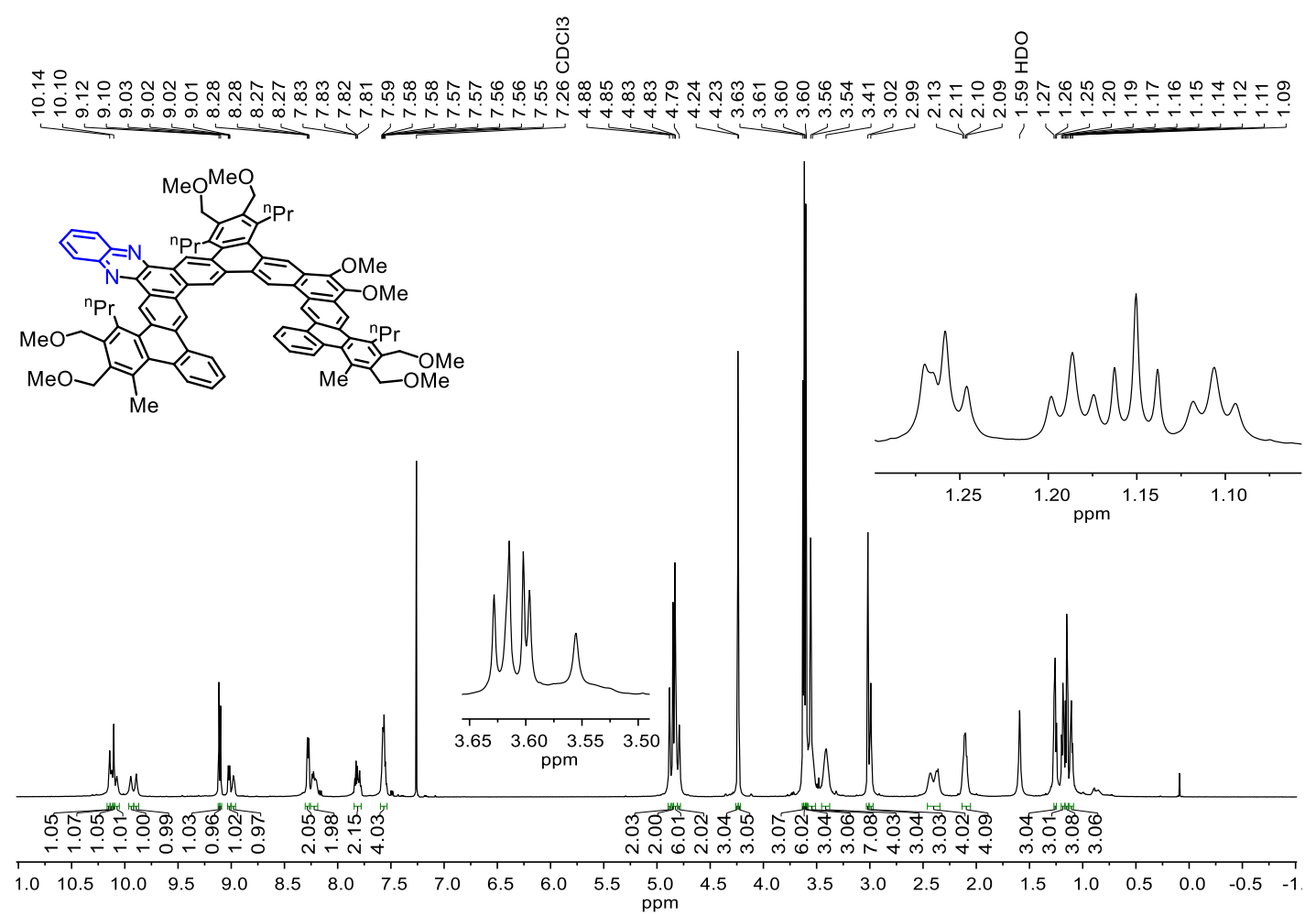

Figure S7. ${ }^{1} \mathrm{H}$ NMR spectrum ( $500 \mathrm{MHz}$, chloroform- $d$, [c] = $62 \mathrm{mM}$ ) of 3-mon.

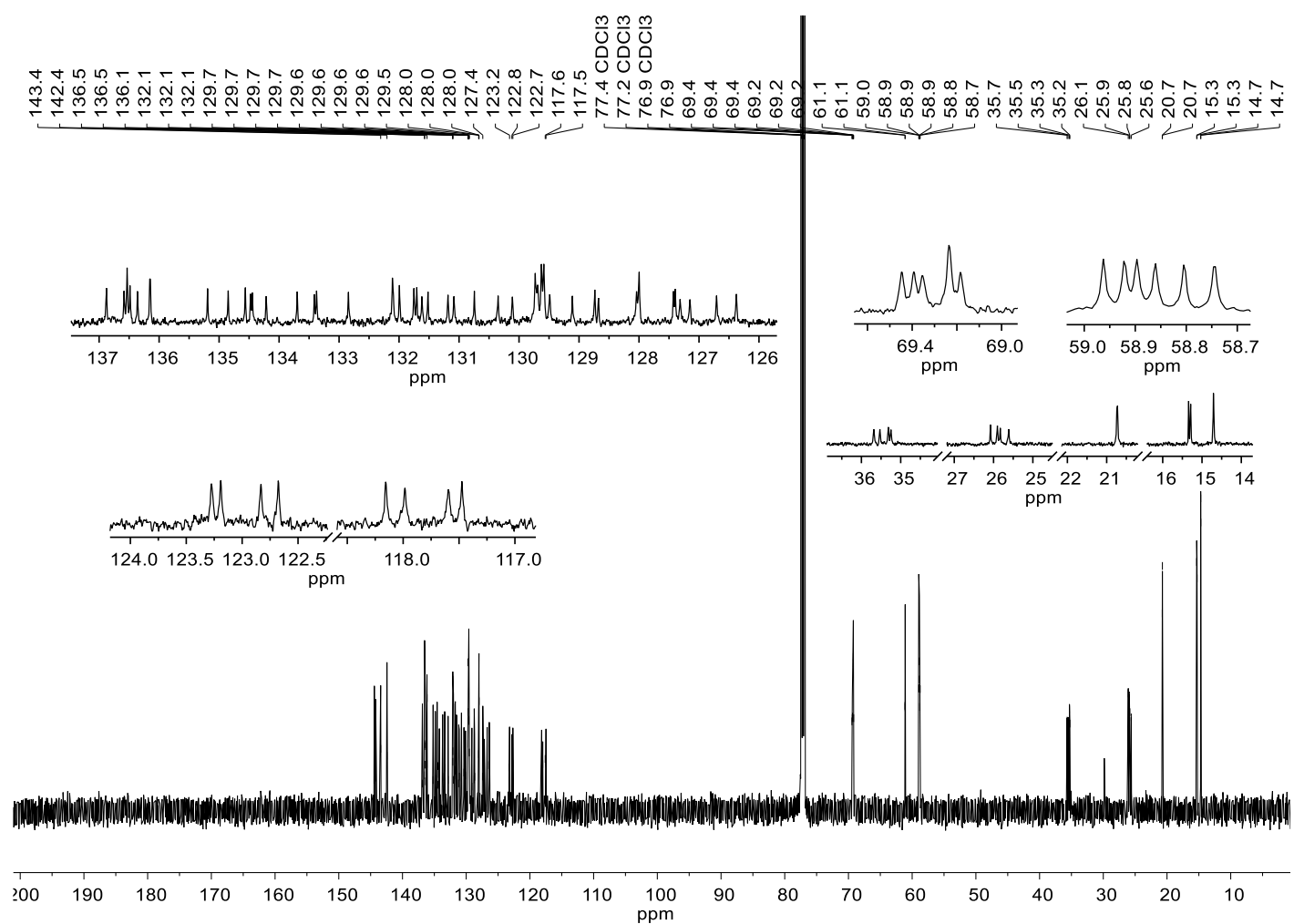

Figure S8. ${ }^{13} \mathrm{C}\left\{{ }^{1} \mathrm{H}\right\}$ NMR spectrum (151 MHz, chloroform- $d$, [c] = $62 \mathrm{mM}$ ) of 3-mon. 


\section{Variable concentration ${ }^{1} \mathrm{H}$ NMR spectra}

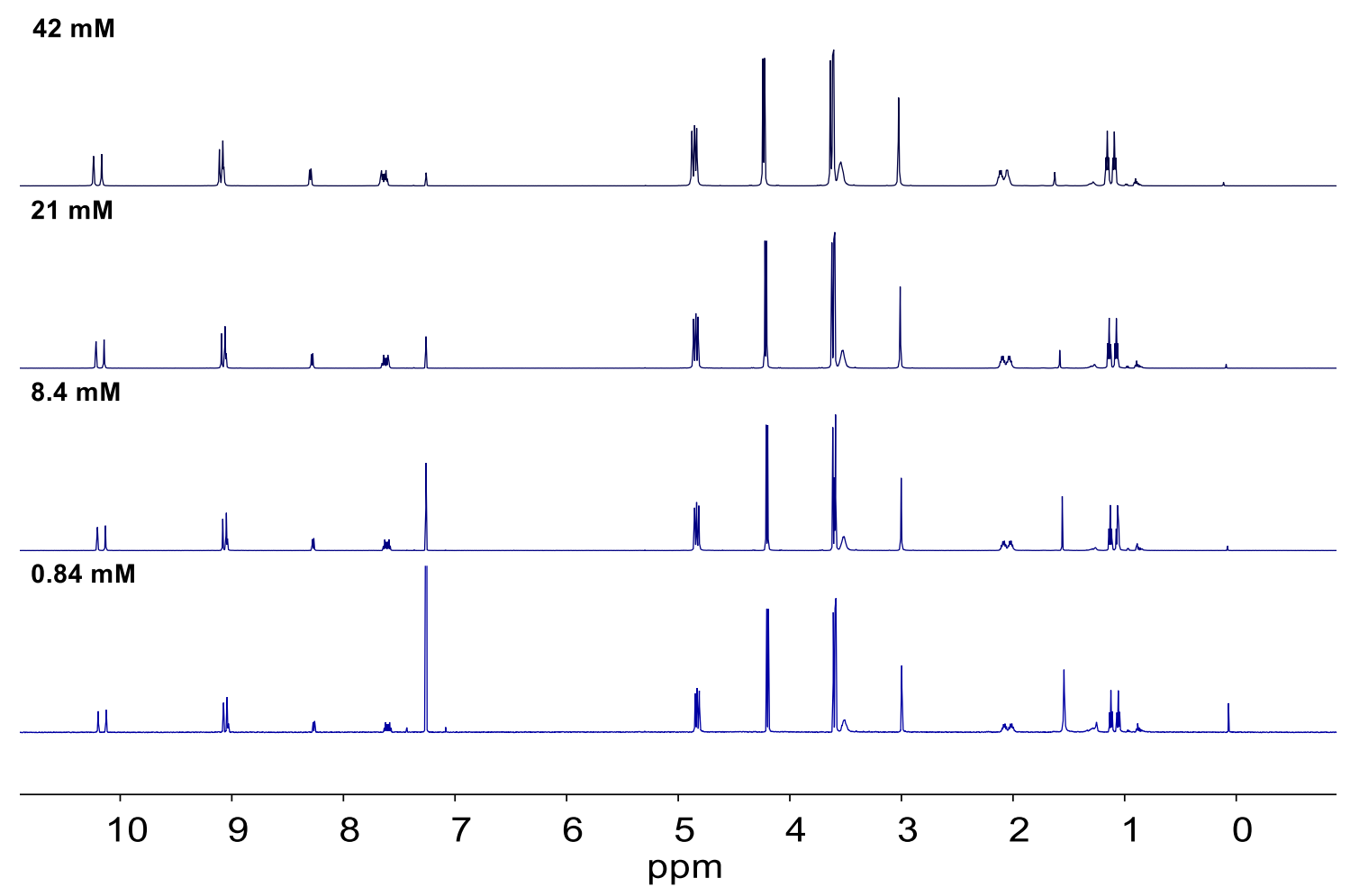

Figure S9. Variable concentration ${ }^{1} \mathrm{H}$ NMR spectra $(600 \mathrm{MHz}$, chloroform- $d)$ of 1a.

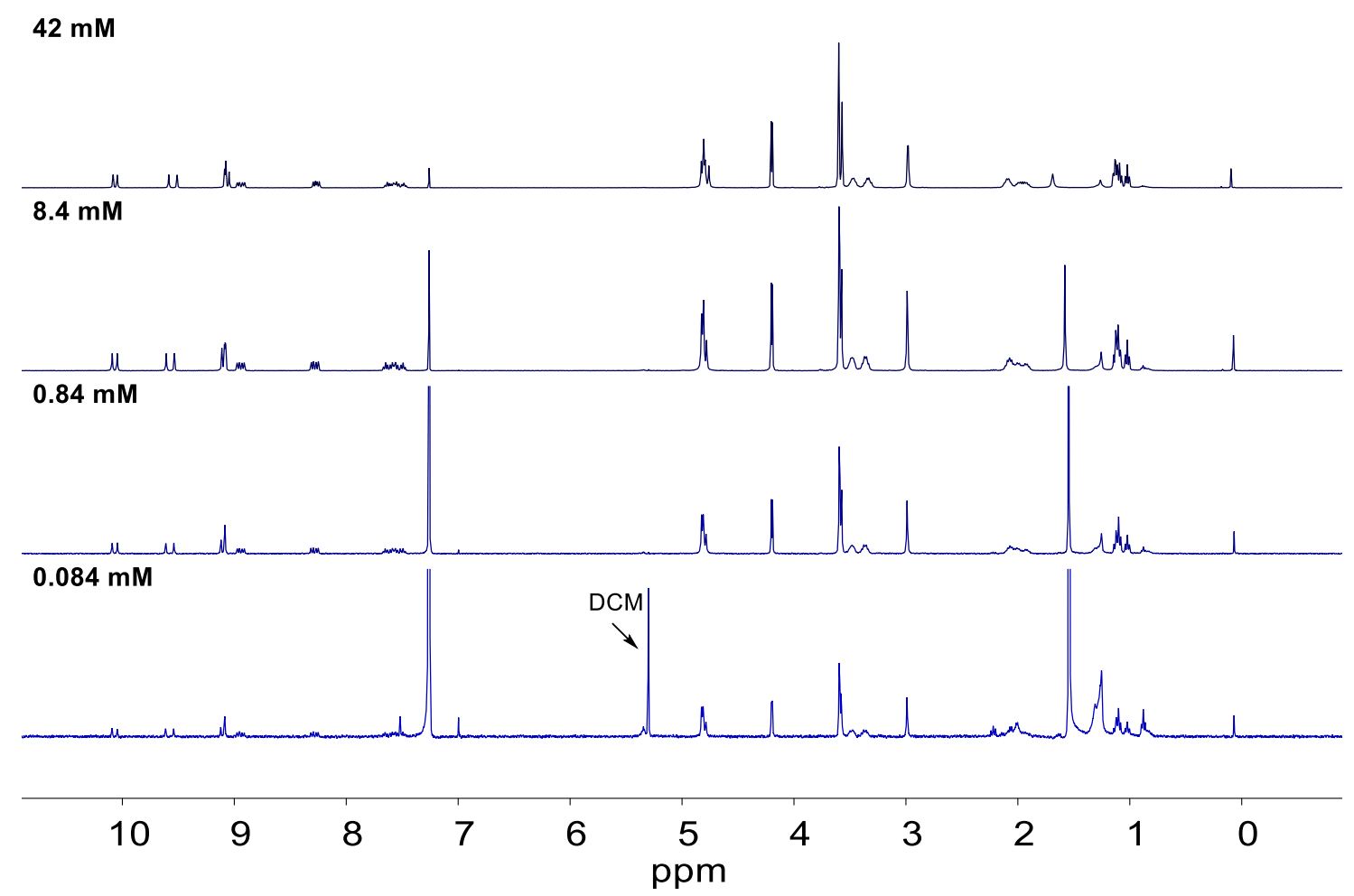

Figure S10. Variable concentration ${ }^{1} \mathrm{H}$ NMR spectra (400 MHz, chloroform- $d$ ) of 2-mon. 


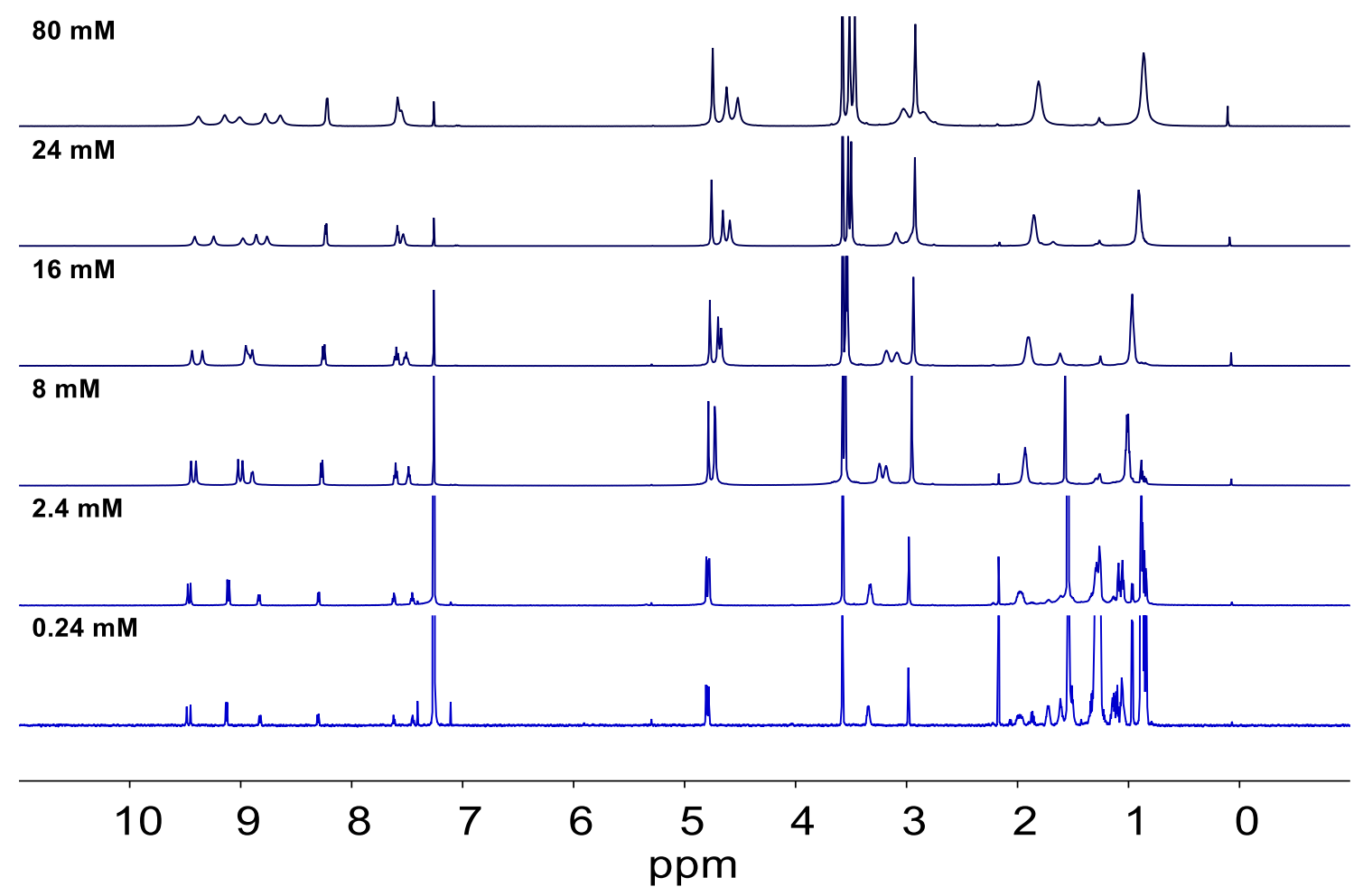

Figure S11. Variable concentration ${ }^{1} \mathrm{H}$ NMR spectra (700 MHz, chloroform- $d$ ) of 2-di.

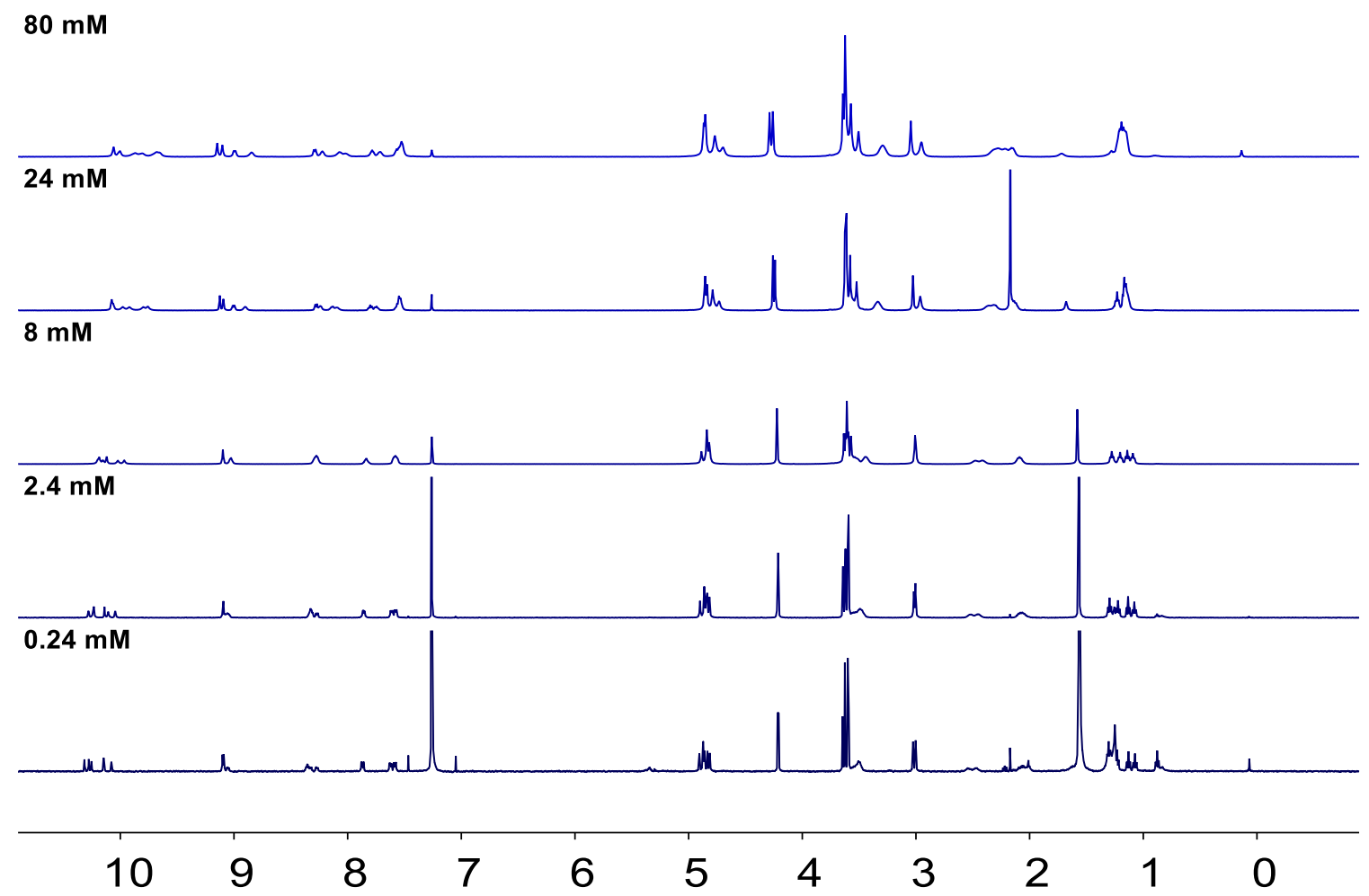

Figure S12. Variable concentration ${ }^{1} \mathrm{H}$ NMR spectra (500 MHz, chloroform-d) of 3-mon. 


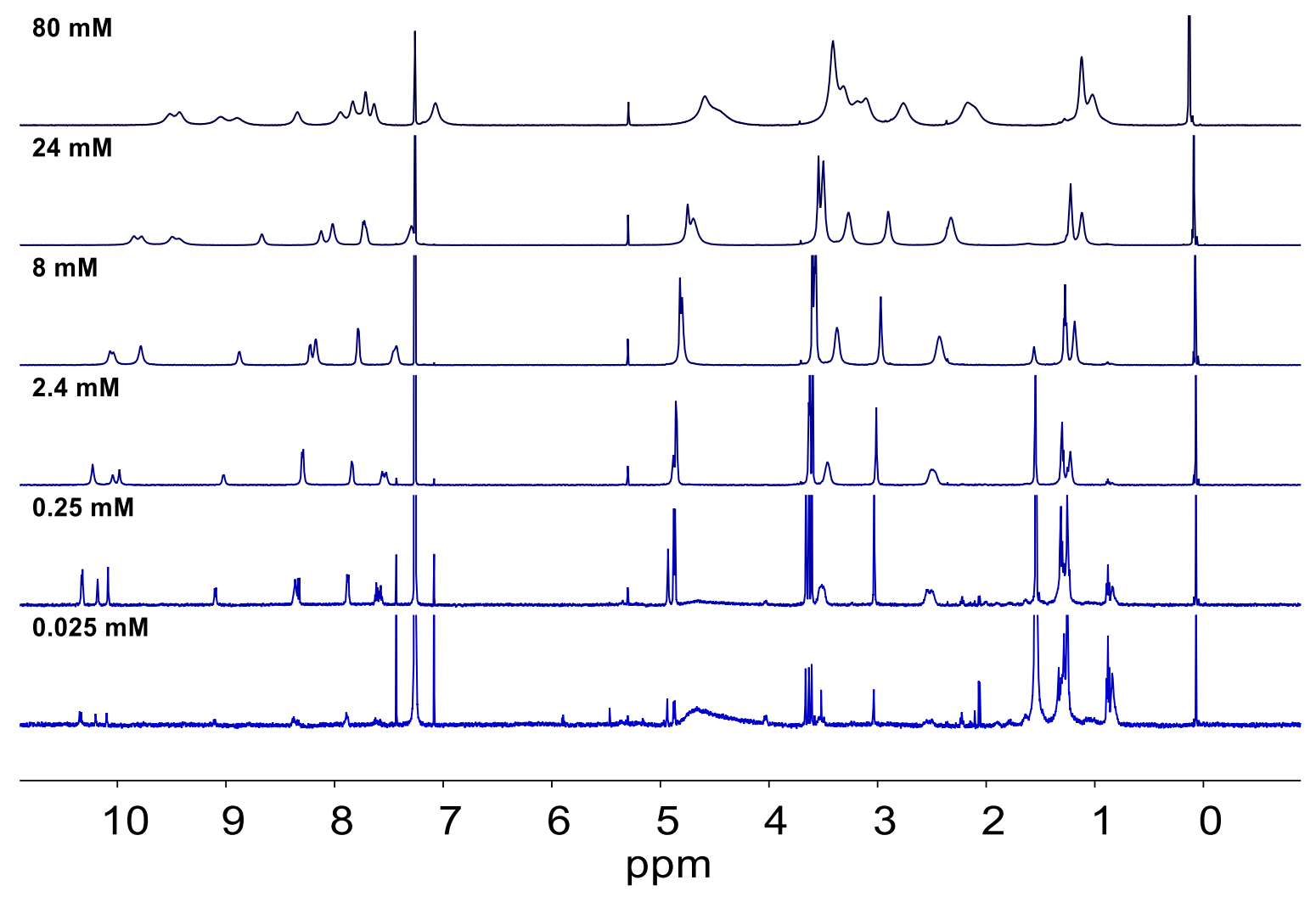

Figure S13. Variable concentration ${ }^{1} \mathrm{H}$ NMR spectra $(600 \mathrm{MHz}$, chloroform- $d)$ of 3-di. 


\section{MicroED experimental details}

\section{Crystallization procedures}

Samples were crystallized using the following procedures:

1a: Approximately $800 \mathrm{mg}$ of 1 a was dissolved in $\mathrm{CH}_{2} \mathrm{Cl}_{2}(6 \mathrm{~mL})$. Hexanes $(10 \mathrm{~mL})$ was added and the total volume reduced to $8 \mathrm{~mL}$, precipitating a yellow microcrystalline solid.

2-di: An approximately $1 \mathrm{mg} / \mathrm{mL}$ solution of 2-di in ethyl acetate was vapor diffused with pentane at room temperature for 24 hours to yield orange needles.

2-mon: An approximately $1 \mathrm{mg} / \mathrm{mL}$ solution of 2-mon in ethyl acetate was vapor diffused with pentane at room temperature for 24 hours to yield orange needles.

3-di: An approximately $1 \mathrm{mg} / \mathrm{mL}$ solution of 3-di in benzene was sonicated for several hours until fully dissolved. The solution was evaporated slowly to yield yellow rods.

3-mon: An approximately $1 \mathrm{mg} / \mathrm{mL}$ solution of 3-mon in benzene was sonicated for several hours and gently heated until sample was fully dissolved. The solution was slow evaporated to yield yellow rods.

\section{Sample preparation for electron diffraction}

Samples were prepared using either Quantifoil or pure Carbon TEM grids. To prepare grids, approximately $2 \mu \mathrm{L}$ of the mother liquor containing crystals were pipetted on to the surface of the TEM grid and excess solvent was wicked away using a Kimwipe. TEM grids were then placed in a Gatan 626 cryo-holder and frozen under liquid nitrogen.

\section{Instrument parameters}

Data for 2-mon, 2-di, 3-mon, and 3-di were acquired on a Thermo Fisher Talos F200C electron microscope operating at an acceleration voltage of $200 \mathrm{KeV}$, corresponding to a wavelength of $0.0251 \AA$. Data for 1 a was acquired on an FEl Tecnai TF-30 electron microscope operating at ambient temperature with and operating voltage of $300 \mathrm{KeV}$, corresponding to a wavelength of $0.0196 \AA$. Screening of the TEM grids for micro crystals was done by operating the microscope in over focused diffraction mode to minimize diffraction and hysteresis between screening and diffraction operational modes.

\section{Electron diffraction data collection procedure}

Electron diffraction data was collected using either Thermo-Fischer CetaD or TVIPS TemCamXF416 CMOS 4k x 4k camera. Images were collected in a movie format as crystals were continuously rotated in the electron beam. ${ }^{3}$ Typical data collection was performed using a constant tilt rate of $0.3^{\circ} \mathrm{s}$ between the minimum and maximum tilt ranges of $-72^{\circ}$ to $+72^{\circ}$, respectively. During continuous rotation the camera integrated frames continuously at a rate of 3 seconds per frame. The dose rate was calibrated to $<0.03 \mathrm{e}^{-} / \AA^{2} \mathrm{~s}$. Crystals selected for data collection were isolated by a selected area aperture to reduce the background noise contributions and calibrated to eucentric height to stay in the aperture over the entire tilt range.

\section{X-ray crystallography}

Synchrotron data for 3-mon was collected on beamline 24-ID-C at the Advanced Photon Source at a wavelength of $0.7749 \AA$, Argonne National Laboratory, which is equipped with a single axis MD2 goniometer, X-ray diffractometer and a Dectris Eiger2 $16 \mathrm{M}$ pixel array detector at a distance of $150 \mathrm{~mm}$. This single axis goniometer limited our achievable value of $\sin$ (theta-max)/wavelength but did not affect the unambiguous determination of this structure. 


\section{Crystallographic data processing}

All diffraction data were processed using the XDS suite of programs. ${ }^{4}$ All structures were solved $a b$ initio using direct methods in SHELXT ${ }^{5}$ or SHELXD ${ }^{6}$ and refined with SHELXL ${ }^{7}$ using ShelXle. ${ }^{8}$ Thermal parameters for 1a, 2-di, 3-di, and 3-mon were refined anisotropically for all non-hydrogen atoms. 2-mon was refined isotropically due to disorder causing a significant portion of the atoms to become non-positive definite. For all structures, hydrogen atoms were assigned using the riding model. Due to inherent disorder within crystals, and the fact that several datasets must be merged to obtain solutions, structures obtained show high $\mathrm{R}$-values and areas of residual density which is difficult to refine for. The data here is the best which could be obtained through electron or synchrotron sources yielding results which would otherwise be unobtainable through standard X-ray analysis. The identity of each compound was verified through spectroscopic analysis; thus, the primary purpose of crystallographic studies was to understand solid state packing characteristics, for which such data is sufficient.

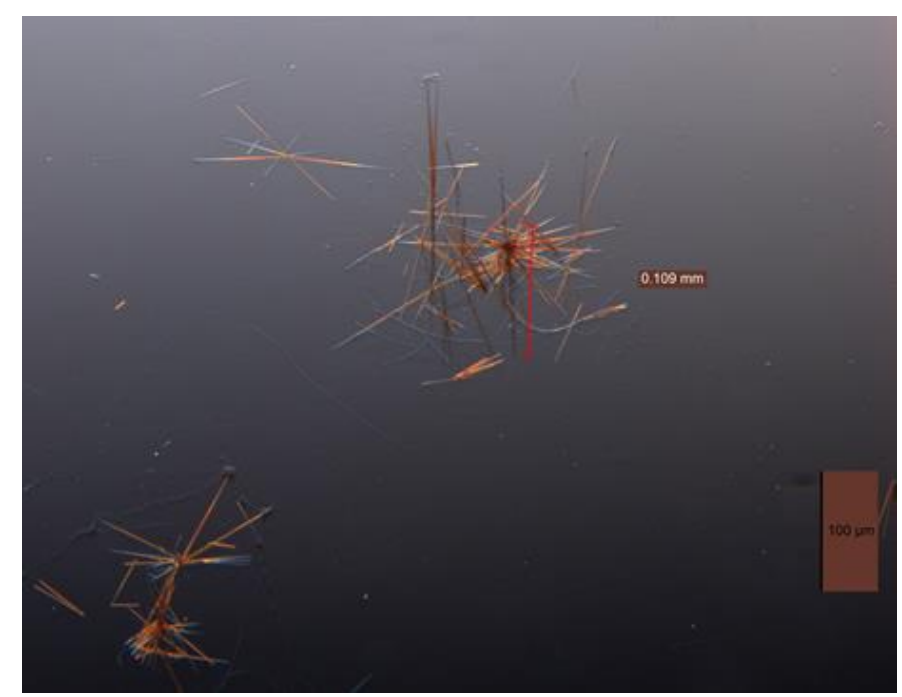

Figure S14. Microscope image of the crystals used to attain the MicroED structure for 2-di.

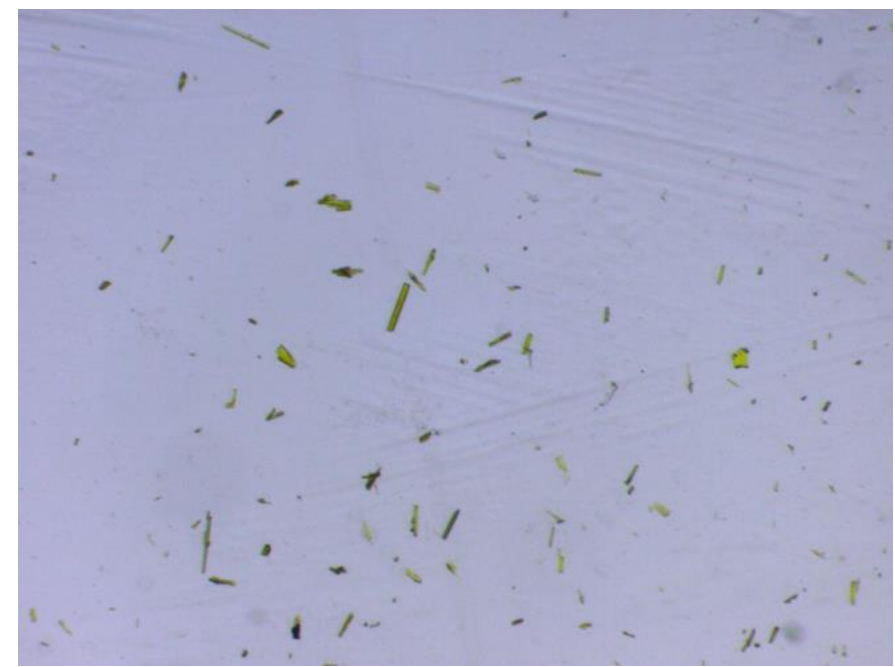

Figure S15. Microscope image of the crystals used to attain the MicroED structure for 3-mon. 


\section{Compound 1a: MicroED data}
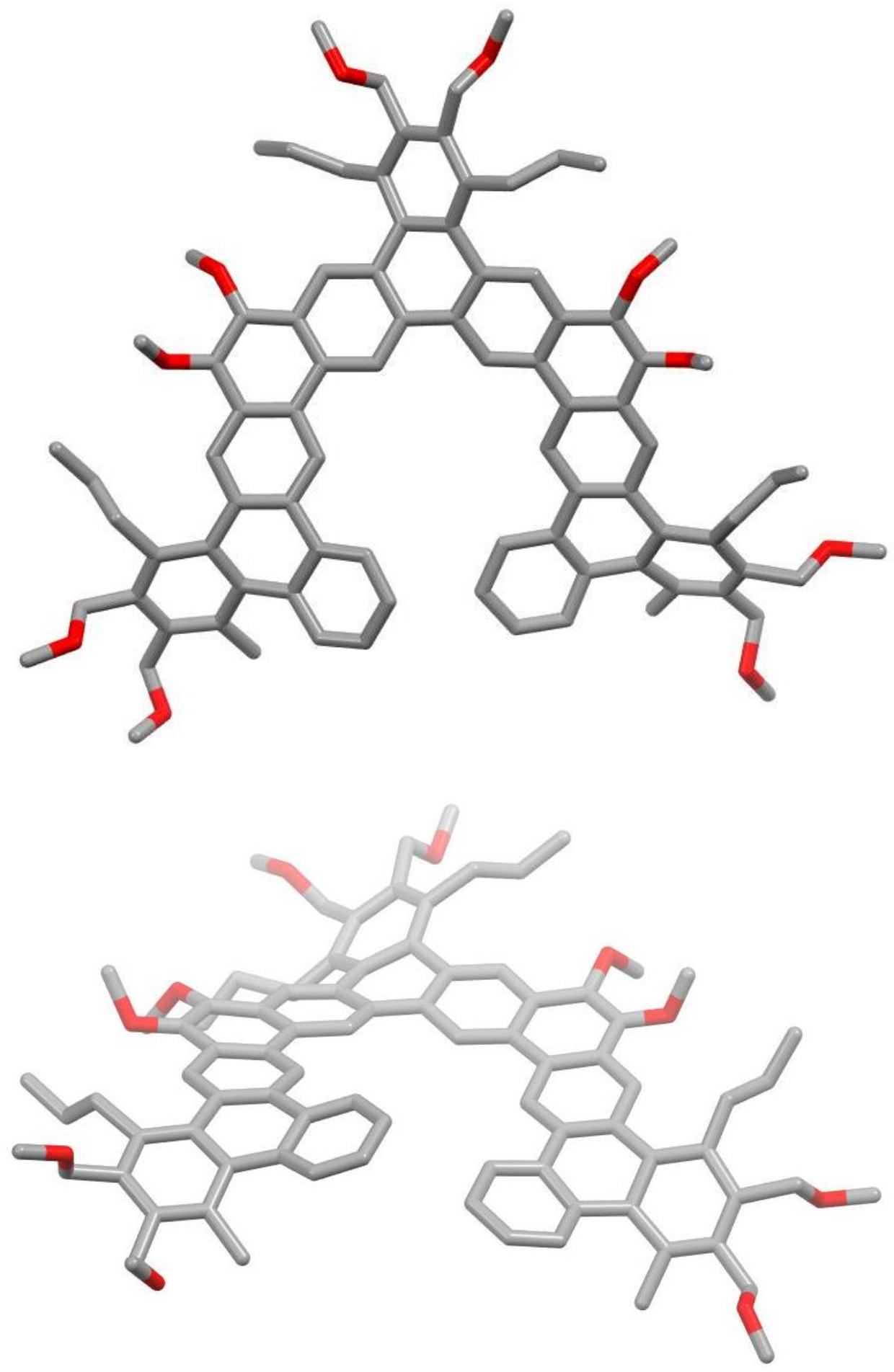

Figure S16. MicroED structure for 1a (CCDC 2026244). 


\begin{tabular}{|c|c|}
\hline Structure & $1 \mathbf{a}$ \\
\hline Empirical formula & $\mathrm{C}_{88} \mathrm{H}_{92} \mathrm{O}_{10}$ \\
\hline Formula weight $(\mathrm{g} / \mathrm{mol})$ & 1279.62 \\
\hline Crystal system & Orthorhombic \\
\hline Space group & $\mathrm{P} 2{ }_{1} 2_{2} 2_{1}(19)$ \\
\hline Point group & 222 \\
\hline Laue symmetry & $\mathrm{mmm}$ \\
\hline Temperature $(\mathrm{K})$ & 298 \\
\hline Unit cell lengths a, b, c $(\dot{A})$ & $11.450(2), 18.420(4), 31.040(6)$ \\
\hline Unit cell angles $\alpha, \beta, \gamma\left(^{\circ}\right)$ & $90.000,90.000,90.000$ \\
\hline Unit cell volume $\left(\AA^{3}\right)$ & $6547(2)$ \\
\hline$F(000)$ & 289 \\
\hline Z & 4 \\
\hline Density (calculated) $\left(\mathrm{g} / \mathrm{cm}^{3}\right)$ & 1.329 \\
\hline Radiation source, wavelength $(\AA)$ & electron, 0.01969 \\
\hline Resolution $(\AA)$ & 1.00 \\
\hline Measured reflections & 31063 \\
\hline Unique reflections & 5846 \\
\hline Reflections with $I>2 \sigma(I)$ & 3977 \\
\hline Completeness & $83.0 \%$ (4 merged datasets) \\
\hline$/ / \sigma$ & 7.38 \\
\hline$\Theta_{\max }, \Theta_{\min }\left({ }^{\circ}\right)$ & $0.57,0.06$ \\
\hline Index ranges & $\begin{array}{l}-11 \leq h \leq 11,-18 \leq k \leq 18 \\
-26 \leq I \leq 26\end{array}$ \\
\hline Refinement method & Full-matrix least-squares on $\mathrm{F}^{2}$ \\
\hline $\mathrm{H}$-atom treatment & $\mathrm{H}$-atom parameters constrained \\
\hline$R\left[F^{2}>2 \sigma\left(F^{2}\right)\right]$ & 0.117 \\
\hline$w R\left(F^{2}\right)$ & 0.264 \\
\hline Goodness-of-fit on $\mathrm{F}^{2}$ & 1.367 \\
\hline$\Delta \rho_{\max }, \Delta \rho_{\min }\left(\mathrm{e} / \dot{\AA}^{3}\right)$ & $0.16,-0.19$ \\
\hline
\end{tabular}

Table S1. MicroED data for 1a (CCDC 2026244). 


\section{Compound 2-mon: MicroED data}
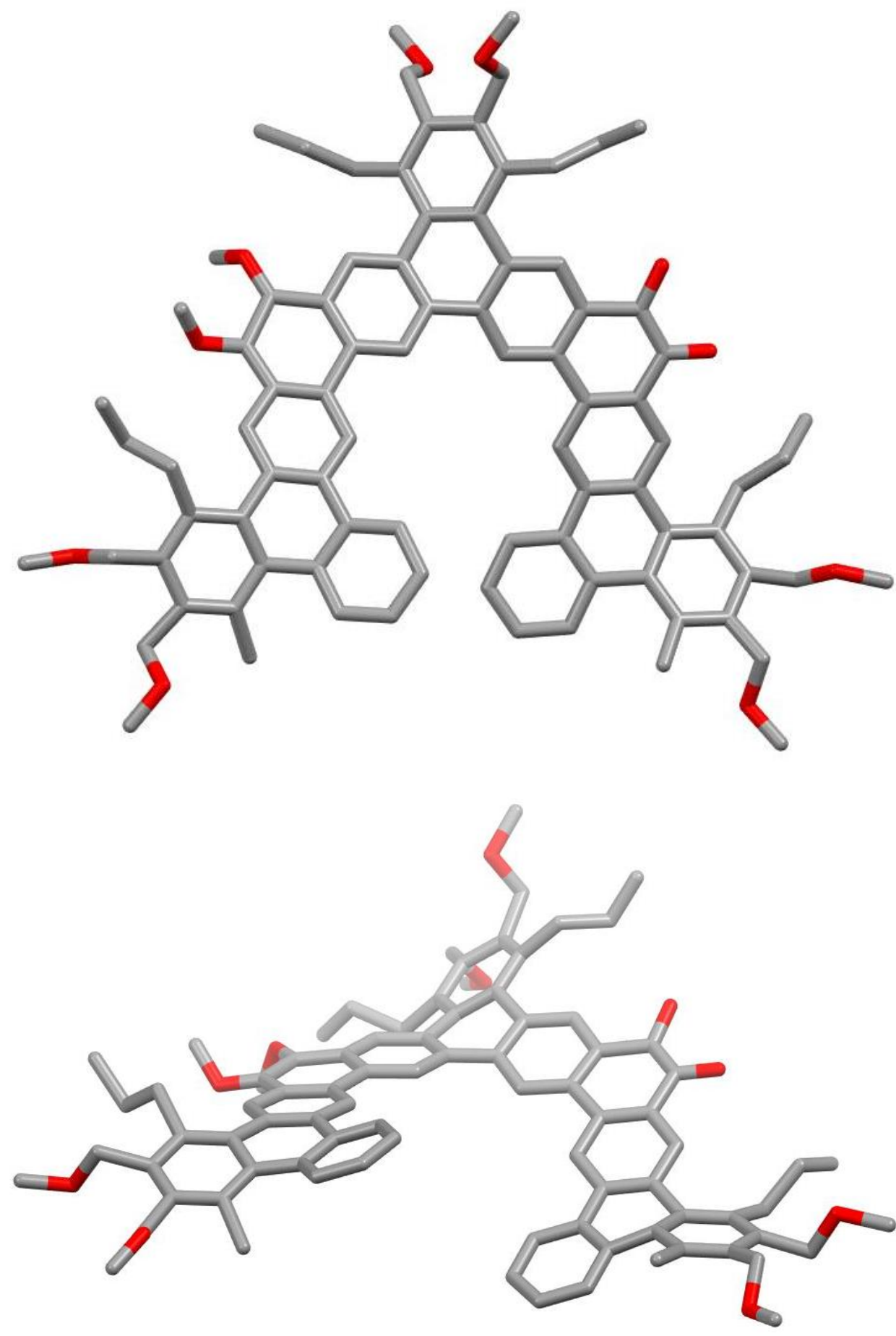

Figure S17. MicroED structure for 2-mon (CCDC 2026247). 


\begin{tabular}{|c|c|}
\hline Structure & 2-mon \\
\hline Empirical formula & $\mathrm{C}_{86} \mathrm{H}_{86} \mathrm{O}_{10}$ \\
\hline Formula weight $(\mathrm{g} / \mathrm{mol})$ & 1279.62 \\
\hline Crystal system & Orthorhombic \\
\hline Space group & Iba2 (45) \\
\hline Point group & $\mathrm{mm2}$ \\
\hline Laue symmetry & $\mathrm{mmm}$ \\
\hline Temperature $(\mathrm{K})$ & 100 \\
\hline Unit cell lengths $a, b, c(\AA)$ & $47.570(2), 47.180(3), 8.190(3)$ \\
\hline Unit cell angles $\alpha, \beta, \gamma\left({ }^{\circ}\right)$ & $90.000,90.000,90.000$ \\
\hline Unit cell volume $\left(\AA^{3}\right)$ & $18381(7)$ \\
\hline$F(000)$ & 281 \\
\hline Z & 8 \\
\hline Density (calculated) $\left(\mathrm{g} / \mathrm{cm}^{3}\right)$ & 0.925 \\
\hline Radiation source, wavelength $(\AA)$ & electron, 0.02508 \\
\hline Resolution $(\AA)$ & 1.10 \\
\hline Measured reflections & 26331 \\
\hline Unique reflections & 6674 \\
\hline Reflections with $I>2 \sigma(I)$ & 4599 \\
\hline Completeness & 90.7\% (3 merged datasets) \\
\hline $1 / \sigma$ & 6.24 \\
\hline$\Theta_{\max }, \Theta_{\min }\left({ }^{\circ}\right)$ & $0.65,0.08$ \\
\hline Index ranges & $\begin{array}{l}-41 \leq h \leq 40,-42 \leq k \leq 42 \\
-7 \leq l \leq 7\end{array}$ \\
\hline Refinement method & Full-matrix least-squares on $\mathrm{F}^{2}$ \\
\hline $\mathrm{H}$-atom treatment & $\mathrm{H}$-atom parameters constrained \\
\hline$R\left[F^{2}>2 \sigma\left(F^{2}\right)\right]$ & 0.163 \\
\hline$w R\left(F^{2}\right)$ & 0.385 \\
\hline Goodness-of-fit on $\mathrm{F}^{2}$ & 1.721 \\
\hline$\Delta \rho_{\max }, \Delta \rho_{\min }\left(\mathrm{e} / \AA^{3}\right)$ & $0.25,-0.26$ \\
\hline
\end{tabular}

Table S2. MicroED data for 2-mon (CCDC 2026247). 


\section{Compound 2-di: MicroED data}
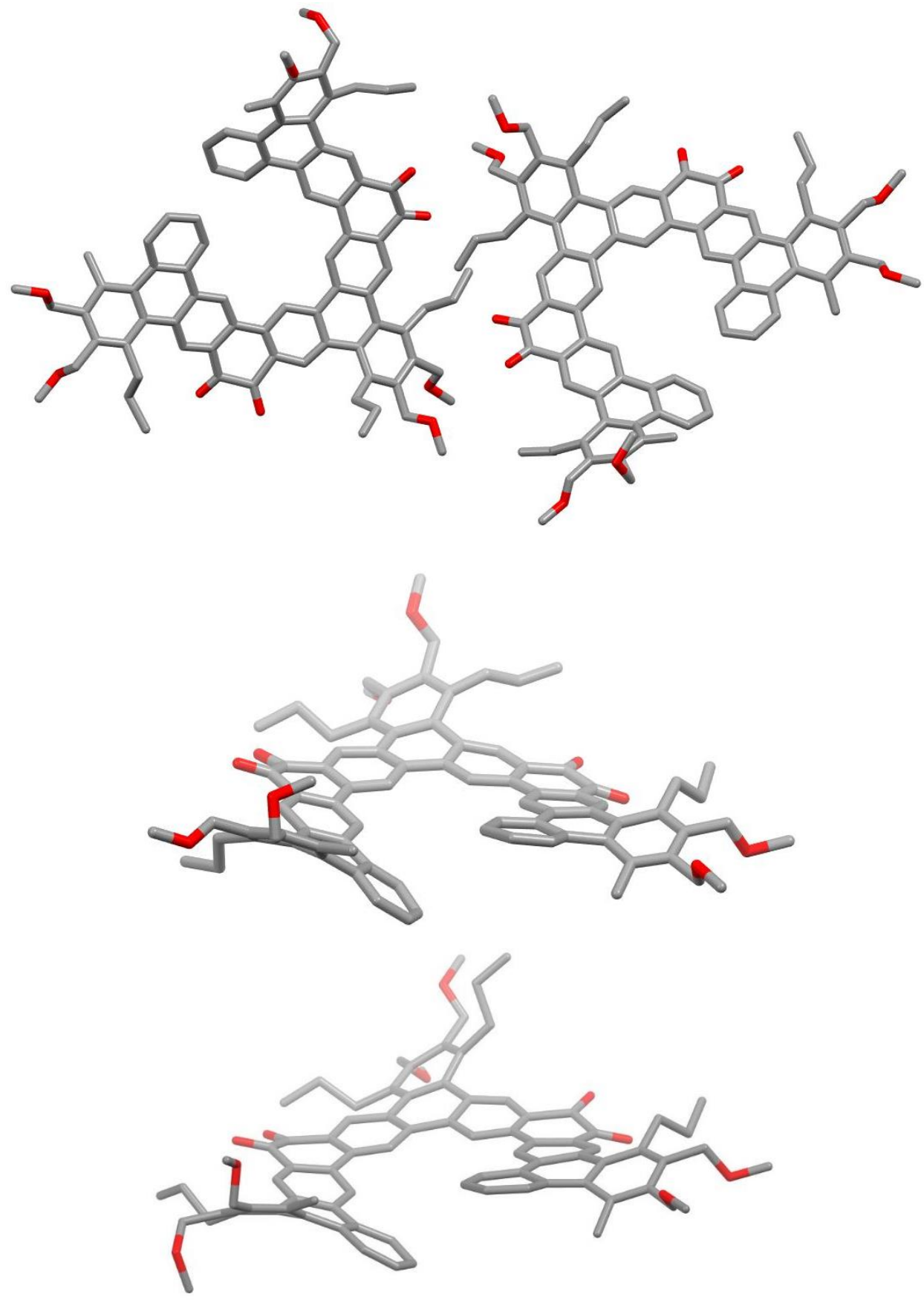

Figure S18. MicroED structure for 2-di (CCDC 2026249). 


\begin{tabular}{|c|c|}
\hline Structure & 2-di \\
\hline Empirical formula & $\mathrm{C}_{84} \mathrm{H}_{80} \mathrm{O}_{10}$ \\
\hline Formula weight $(\mathrm{g} / \mathrm{mol})$ & 1249.55 \\
\hline Crystal system & Triclinic \\
\hline Space group & $\mathrm{P}-1(2)$ \\
\hline Point group & -1 \\
\hline Laue symmetry & -1 \\
\hline Temperature $(\mathrm{K})$ & 100 \\
\hline Unit cell lengths a, b, c $(\dot{A})$ & $8.030(2), 18.610(4), 45.130(9)$ \\
\hline Unit cell angles $\alpha, \beta, \gamma\left(^{\circ}\right)$ & $81.18(3), 89.13(3), 84.05(3)$ \\
\hline Unit cell volume $\left(\AA^{3}\right)$ & $6629(3)$ \\
\hline$F(000)$ & 296 \\
\hline Z & 4 \\
\hline Density (calculated) $\left(\mathrm{g} / \mathrm{cm}^{3}\right)$ & 1.324 \\
\hline Radiation source, wavelength $(\AA)$ & electron, 0.02508 \\
\hline Resolution $(\AA)$ & 1.00 \\
\hline Measured reflections & 45615 \\
\hline Unique reflections & 9966 \\
\hline Reflections with $I>2 \sigma(I)$ & 6597 \\
\hline Completeness & $72.0 \%$ (4 merged datasets) \\
\hline$/ / \sigma$ & 5.66 \\
\hline$\Theta_{\max }, \Theta_{\min }\left({ }^{\circ}\right)$ & $0.72,0.08$ \\
\hline Index ranges & $\begin{array}{l}-8 \leq h \leq 8,-16 \leq k \leq 16, \\
-45 \leq I \leq 45\end{array}$ \\
\hline Refinement method & Full-matrix least-squares on $\mathrm{F}^{2}$ \\
\hline $\mathrm{H}$-atom treatment & $\mathrm{H}$-atom parameters constrained \\
\hline$R\left[F^{2}>2 \sigma\left(F^{2}\right)\right]$ & 0.172 \\
\hline$w R\left(F^{2}\right)$ & 0.437 \\
\hline Goodness-of-fit on $\mathrm{F}^{2}$ & 1.588 \\
\hline$\Delta \rho_{\max }, \Delta \rho_{\min }\left(\mathrm{e} / \dot{\AA}^{3}\right)$ & $0.24,-0.20$ \\
\hline
\end{tabular}

Table S3. MicroED data for 2-di (CCDC 2026249). 
Compound 3-mon: MicroED and X-ray data
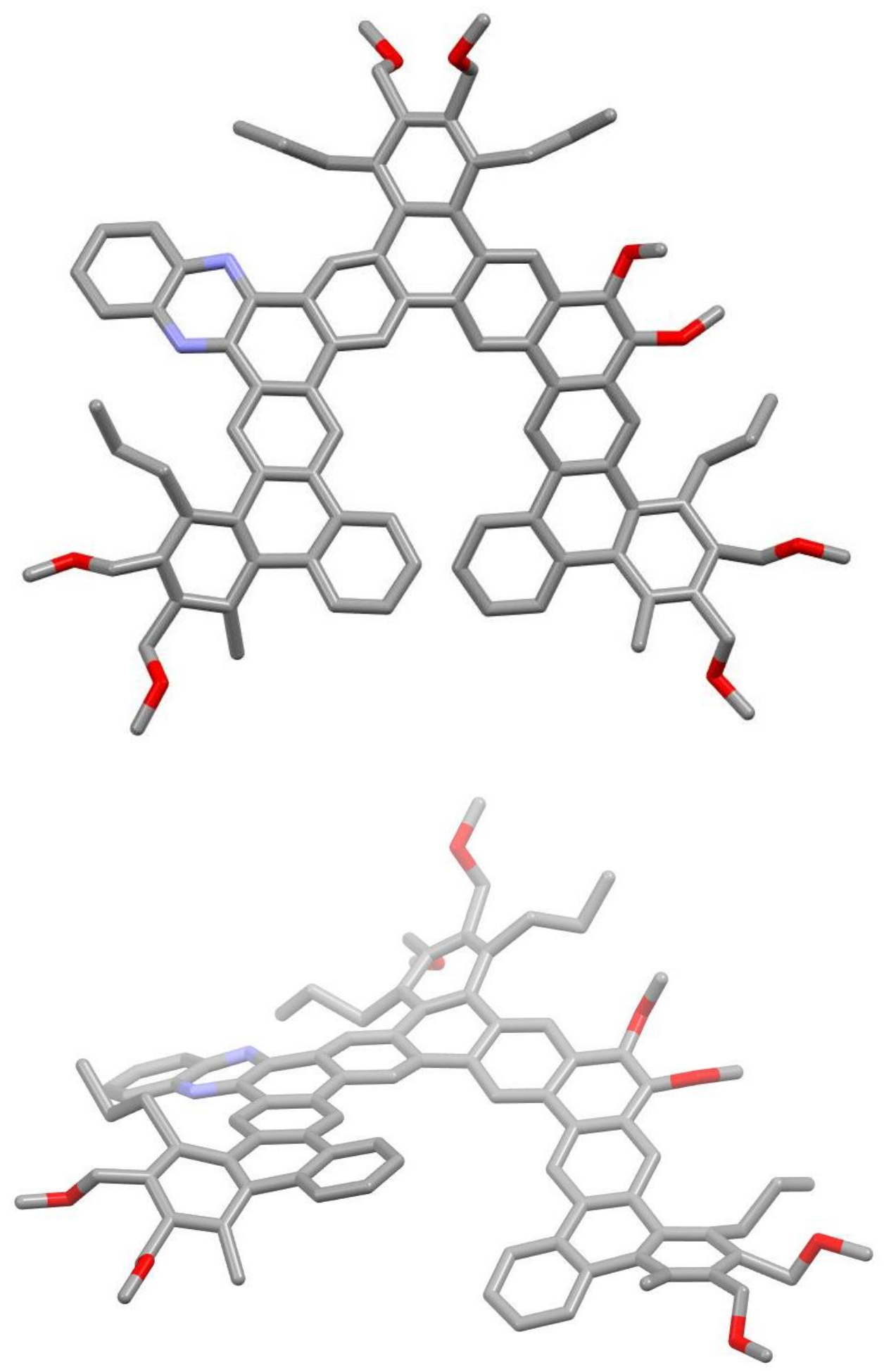

Figure S19. MicroED structure for 3-mon (CCDC 2026252). 


\begin{tabular}{|c|c|c|}
\hline Structure & 3-mon (electron) & 3-mon (synchrotron) \\
\hline Empirical formula & $\mathrm{C}_{92} \mathrm{H}_{90} \mathrm{~N}_{2} \mathrm{O}_{8}$ & $\mathrm{C}_{92} \mathrm{H}_{90} \mathrm{~N}_{2} \mathrm{O}_{8}$ \\
\hline Formula weight $(\mathrm{g} / \mathrm{mol})$ & 1351.74 & 1351.74 \\
\hline Crystal system & Orthorhombic & Orthorhombic \\
\hline Space group & Iba2 (45) & Iba2 (45) \\
\hline Point group & $\mathrm{mm} 2$ & $\mathrm{~mm} 2$ \\
\hline Laue symmetry & $\mathrm{mmm}$ & $\mathrm{mmm}$ \\
\hline Temperature $(\mathrm{K})$ & 100 & 100 \\
\hline Unit cell lengths a, b, c $(\AA)$ & 48.29(5), 46.86(5), 7.970(8) & $\begin{array}{ll}47.750(2), & 47.700(4), \\
8.220(6) & \\
\end{array}$ \\
\hline Unit cell angles $\alpha, \beta, \gamma\left(\left(^{\circ}\right)\right.$ & $90.000,90.000,90.000$ & $90.000,90.000,90.000$ \\
\hline Unit cell volume $\left(\AA^{3}\right)$ & $18035(32)$ & $18723(14)$ \\
\hline$F(000)$ & 295 & 720 \\
\hline Z & 8 & 8 \\
\hline Density (calculated) $\left(\mathrm{g} / \mathrm{cm}^{3}\right)$ & 0.996 & 0.959 \\
\hline $\begin{array}{l}\text { Radiation source, wavelength } \\
(\AA)\end{array}$ & electron, 0.02508 & synchrotron, 0.77490 \\
\hline Resolution $(\AA)$ & 1.00 & 1.05 \\
\hline Measured reflections & 28118 & 111732 \\
\hline Unique reflections & 9108 & 7747 \\
\hline Reflections with $I>2 \sigma(I)$ & 4033 & 6600 \\
\hline Completeness & $96.8 \%$ (3 merged datasets) & $91.1 \%$ (1 dataset) \\
\hline $1 / \sigma$ & 3.06 & 30.12 \\
\hline$\Theta_{\max }, \Theta_{\min }\left({ }^{\circ}\right)$ & $0.72,0.08$ & $21.68,2.37$ \\
\hline Index ranges & $\begin{array}{l}-46 \leq h \leq 46,-46 \leq k \leq 46, \\
-7 \leq I \leq 7\end{array}$ & $\begin{array}{l}-45 \leq h \leq 45,-42 \leq k \leq 42, \\
-7 \leq I \leq 7\end{array}$ \\
\hline Refinement method & $\begin{array}{l}\text { Full-matrix least-squares on } \\
\mathrm{F}^{2}\end{array}$ & $\begin{array}{l}\text { Full-matrix least-squares on } \\
\mathrm{F}^{2}\end{array}$ \\
\hline $\mathrm{H}$-atom treatment & $\begin{array}{l}\mathrm{H} \text {-atom } \\
\text { constrained }\end{array}$ & $\begin{array}{l}\mathrm{H} \text {-atom } \\
\text { constrained }\end{array}$ \\
\hline$R\left[F^{2}>2 \sigma\left(F^{2}\right)\right]$ & 0.199 & 0.146 \\
\hline$w R\left(F^{2}\right)$ & 0.492 & 0.357 \\
\hline Goodness-of-fit on $\mathrm{F}^{2}$ & 1.280 & 2.968 \\
\hline$\Delta \rho_{\max }, \Delta \rho_{\min }\left(\mathrm{e} / \dot{\mathrm{A}}^{3}\right)$ & $0.21,-0.21$ & $0.59,-0.44$ \\
\hline
\end{tabular}

Table S4. MicroED (CCDC 2026252) and synchrotron (CCDC 2026253) data for 3-mon. 


\section{Compound 3-di: MicroED data}
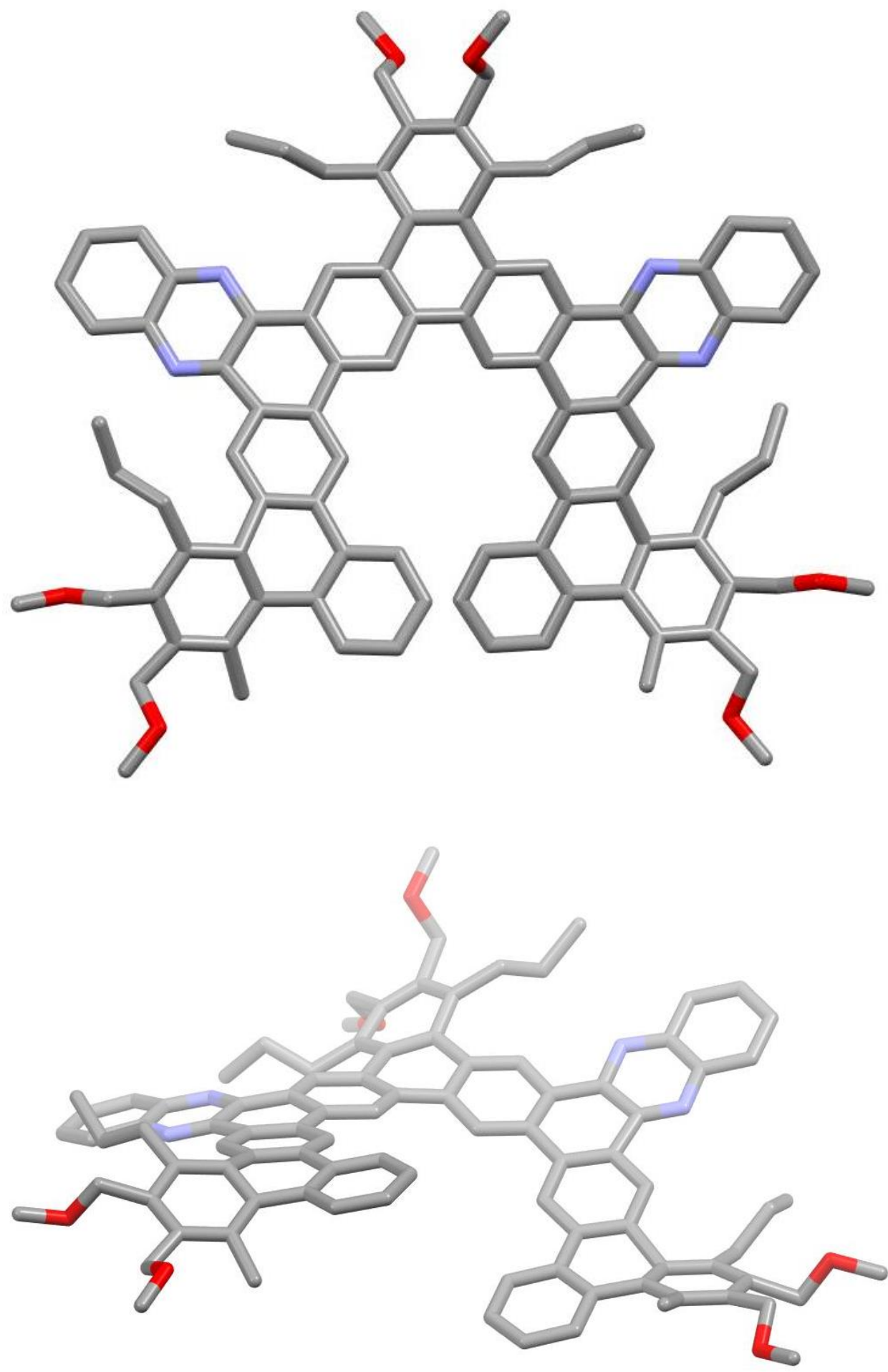

Figure S20. MicroED structure for 3-di (CCDC 2026251). 


\begin{tabular}{|c|c|}
\hline Structure & 3-di \\
\hline Empirical formula & $\mathrm{C}_{96} \mathrm{H}_{88} \mathrm{~N}_{4} \mathrm{O}_{6}$ \\
\hline Formula weight $(\mathrm{g} / \mathrm{mol})$ & 1393.78 \\
\hline Crystal system & Tetragonal \\
\hline Space group & $\mathrm{I}-4 \mathrm{c} 2(120)$ \\
\hline Point group & $-42 m$ \\
\hline Laue symmetry & $4 / \mathrm{mmm}$ \\
\hline Temperature $(\mathrm{K})$ & 100 \\
\hline Unit cell lengths $a, b, c(\AA)$ & $47.150(3), 47.150(3), 7.980(9)$ \\
\hline Unit cell angles $\alpha, \beta, \gamma\left(^{\circ}\right)$ & $90.000,90.000,90.000$ \\
\hline Unit cell volume $\left(\dot{A}^{3}\right)$ & $17741(20)$ \\
\hline$F(000)$ & 308 \\
\hline Z & 8 \\
\hline Density (calculated) $\left(\mathrm{g} / \mathrm{cm}^{3}\right)$ & 1.044 \\
\hline Radiation source, wavelength $(\AA)$ & electron, 0.02508 \\
\hline Resolution $(\AA)$ & 1.00 \\
\hline Measured reflections & 10591 \\
\hline Unique reflections & 4390 \\
\hline Reflections with $I>2 \sigma(I)$ & 1917 \\
\hline Completeness & $93.3 \%$ (1 dataset) \\
\hline $1 / \sigma$ & 3.37 \\
\hline$\Theta_{\max }, \Theta_{\min }\left({ }^{\circ}\right)$ & $0.72,0.08$ \\
\hline Index ranges & $\begin{array}{l}-34 \leq h \leq 35,-47 \leq k \leq 46, \\
-7 \leq l \leq 7\end{array}$ \\
\hline Refinement method & Full-matrix least-squares on $\mathrm{F}^{2}$ \\
\hline $\mathrm{H}$-atom treatment & $\mathrm{H}$-atom parameters constrained \\
\hline$R\left[F^{2}>2 \sigma\left(F^{2}\right)\right]$ & 0.171 \\
\hline$w R\left(F^{2}\right)$ & 0.416 \\
\hline Goodness-of-fit on $\mathrm{F}^{2}$ & 1.380 \\
\hline$\Delta \rho_{\max }, \Delta \rho_{\min }\left(\mathrm{e} / \AA^{3}\right)$ & $0.18,-0.18$ \\
\hline
\end{tabular}

Table S5. MicroED Data for 3-di (CCDC 2026251). 


\section{Photophysical characterization}

Ultraviolet/visible (UV/Vis) absorption spectra were recorded on a Varian Cary 300 Bio UV-Visible spectrophotometer. Emission spectra were recorded on a Varian Cary Eclipse Spectrometer, and all compounds were excited at $335 \mathrm{~nm}$. All compounds were prepared as described in the manuscript and used directly. For sample preparation, each analyte was weighed on an analytical balance and dissolved in the appropriate amount of spectroscopy-grade $\mathrm{CH}_{2} \mathrm{Cl}_{2}$ using a volumetric flask. All spectra are presented in the manuscript.

\begin{tabular}{|c|c|c|c|c|c|}
\hline Compound & $\begin{array}{c}\text { Concentration } \\
(\mu \mathrm{M})\end{array}$ & $\begin{array}{c}\text { Absorption } \\
\text { maximum, } \\
\lambda_{\max }(\mathrm{nm})\end{array}$ & $\begin{array}{c}\text { Absorption } \\
\text { onset, } \\
\lambda_{\text {onset }}(\mathrm{nm})^{\mathrm{a}}\end{array}$ & $\begin{array}{c}\text { Emission } \\
\text { Maximum, } \\
\lambda_{\max }(\mathrm{nm})\end{array}$ & $\begin{array}{c}\text { Photophysical } \\
\text { HOMO- } \\
\text { LUMO gap, } \\
\mathrm{E}_{\mathrm{g}}(\mathrm{eV})^{\mathrm{b}}\end{array}$ \\
\hline 1a & 16 & 324 & 445 & 446 & 2.79 \\
\hline 2-mon & 13 & 333 & 595 & ${\mathrm{~N} / \mathrm{A}^{\mathrm{c}}}^{2}$ & 2.08 \\
\hline 2-di & 16 & 339 & 604 & $\mathrm{~N}^{\mathrm{c}} \mathrm{A}^{\mathrm{c}}$ & 2.05 \\
\hline 3-mon & 15 & 319 & 471 & 533 & 2.63 \\
\hline 3-di & 14 & 315 & 471 & 517 & 2.63 \\
\hline
\end{tabular}

Table S6. Summary of relevant photophysical properties in $\mathrm{CH}_{2} \mathrm{Cl}_{2}$ solvent. (a) Defined as $\varepsilon=$ $1000 \mathrm{M}^{-1} \mathrm{~cm}^{-1}$; (b) Estimated from the absorption onset $\left(\lambda_{\text {onset }}\right)$ : $E_{g}=1240 / \lambda_{\text {onset }}$; (c) Nonfluorescent.

\section{Electrochemical characterization}

Cyclic and square wave voltammetry were performed on a BASi EC Epsilon potentiostat/galvanostat with a PWR-3 Power Module using dry, oxygen-free solvents, a glassy carbon working electrode, Pt counter electrode, and $\mathrm{Ag} / \mathrm{Ag}^{+}$reference electrode.

\begin{tabular}{|c|c|c|c|c|c|}
\hline Compound & $\begin{array}{l}\mathrm{E}_{1 / 2}^{\text {red }} v s . \\
\mathrm{Fc} / \mathrm{FC}^{+}(\mathrm{V})^{\mathrm{a}}\end{array}$ & $\begin{array}{c}\mathrm{E}_{1 / 2}{ }^{\mathrm{ox}} \mathrm{vs} \\
\mathrm{Fc} / \mathrm{Fc}^{+}(\mathrm{V})^{\mathrm{a}}\end{array}$ & $\begin{array}{c}\text { Electrochemical } \\
\text { HOMO_LUMO } \\
\text { gap, } \mathrm{E}_{\mathrm{g}}(\mathrm{eV})^{\mathrm{b}}\end{array}$ & $\begin{array}{l}\text { LUMO } \\
(\mathrm{eV})^{\mathrm{c}}\end{array}$ & $\begin{array}{l}\mathrm{HOMO} \\
(\mathrm{eV})^{\mathrm{d}}\end{array}$ \\
\hline $1 a$ & $\mathrm{~N} / \mathrm{A}$ & $0.66,0.75$ & $N / A$ & $-2.67^{e}$ & -5.46 \\
\hline 2-mon & $-1.15,-1.81$ & $0.79,0.98$ & 1.94 & -3.65 & -5.59 \\
\hline 2-di & $\begin{array}{c}-1.08,-1.22 \\
-1.81\end{array}$ & $\mathrm{~N} / \mathrm{A}$ & $\mathrm{N} / \mathrm{A}$ & -3.72 & $-5.77^{e}$ \\
\hline 3-mon & -1.84 & $0.73,0.97$ & 2.57 & -2.96 & -5.53 \\
\hline 3-di & $-1.85,-2.56$ & N/A & $\mathrm{N} / \mathrm{A}$ & -2.95 & $-5.58^{\mathrm{e}}$ \\
\hline
\end{tabular}

Table S7. Summary of relevant electrochemical properties. (a) Reported values are for $\mathrm{E}_{1 / 2}$ were measured with cyclic and square wave voltammetry in $\mathrm{CH}_{2} \mathrm{Cl}_{2}$ with $0.1 \mathrm{M}{ }^{n} \mathrm{Bu}_{4} \mathrm{NPF}_{6}$ as the supporting electrolyte. Compounds that displayed no events for a given window are marked with "N/A"; (b) $E_{g}=E_{1 / 2}{ }^{o x}-E_{1 / 2}{ }^{\text {red; }}$ (c) estimated using first reduction: $-\left(E_{1 / 2}{ }^{\text {red }}+4.80\right)$; (d) estimated using first oxidation: $-\left(E_{1 / 2}{ }^{\text {ox }}+4.80\right)$; (e) When $E_{1 / 2}^{\text {red }}$ or $E_{1 / 2}{ }^{\text {ox }}$ were not available, these values were calculated using a combination of the photophysical $E_{g}$ and the available HOMO or LUMO energy level. 


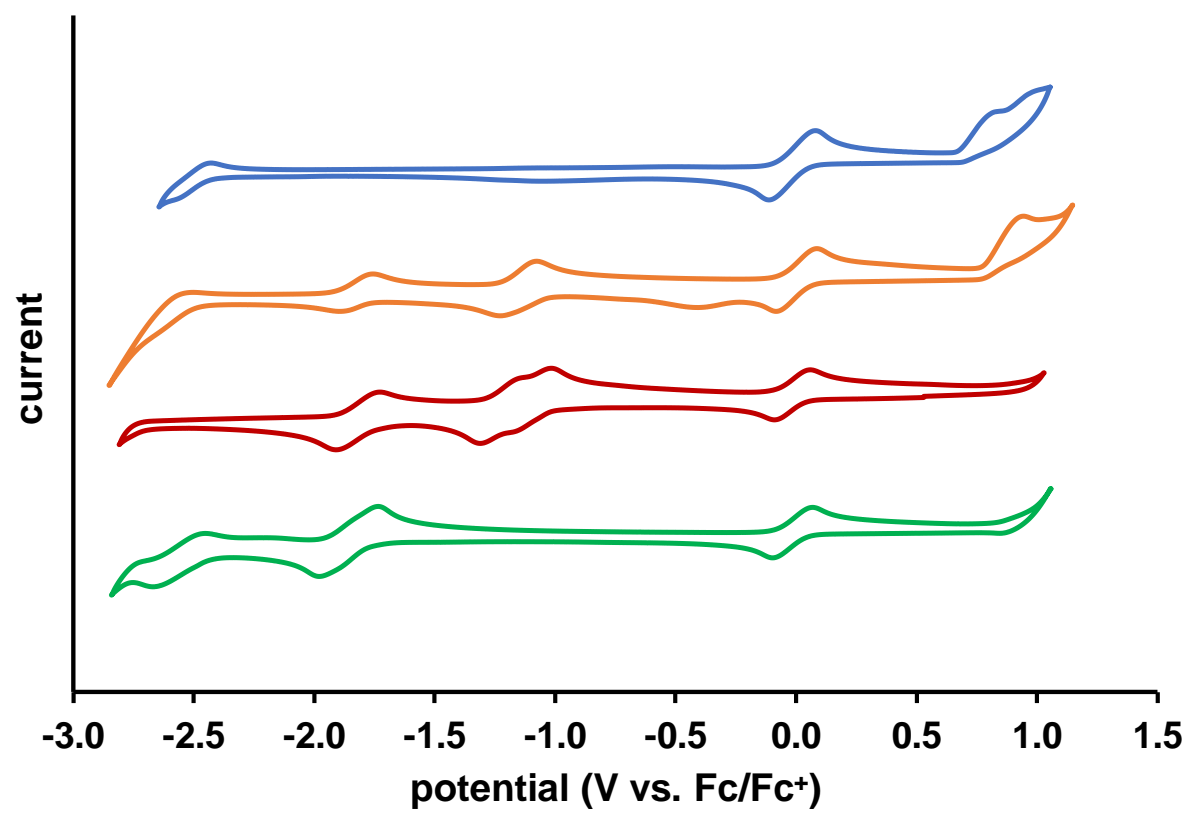

Figure S21. Cyclic voltammograms of compounds 1a, 2-mon, 2-di, 3-di (from top to bottom) in

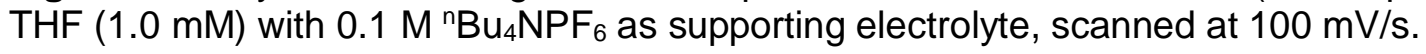

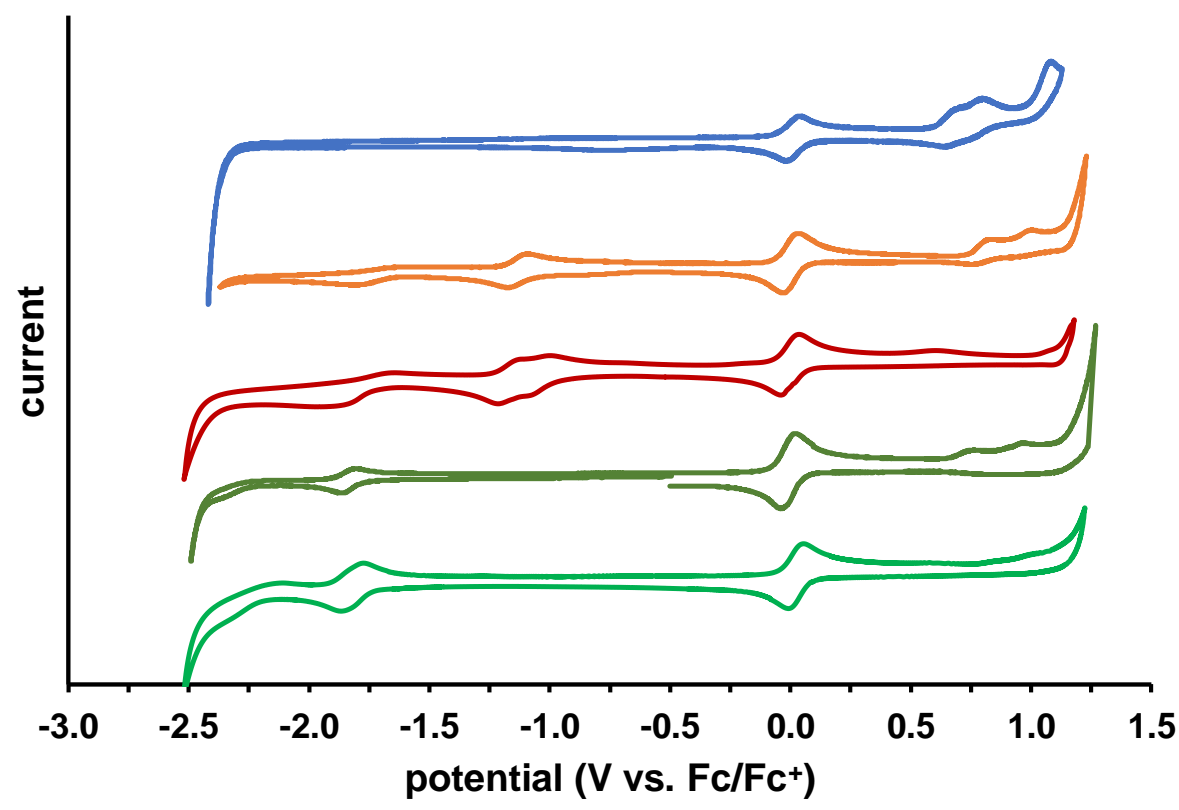

Figure 22. Cyclic voltammograms of compounds 1a, 2-mon, 2-di, 3-mon, 3-di (from top to bottom) in $\mathrm{CH}_{2} \mathrm{Cl}_{2}(1.0 \mathrm{mM})$ with $0.1 \mathrm{M}^{\mathrm{n}} \mathrm{Bu}_{4} \mathrm{NPF}_{6}$ as supporting electrolyte, scanned at $100 \mathrm{mV} / \mathrm{s}$. 


\section{Dynamic light scattering (DLS) characterization}

Samples were analyzed using a ZetaPALS Zeta Potential Analyzer by Brookhaven Instruments, equipped for DLS measurements. Samples were freshly prepared in $\mathrm{CDCl}_{3}$ and filtered through a $0.4 \mu \mathrm{m}$ Teflon filter directly into a quartz cuvette before analysis. The sample holder was maintained at $25^{\circ} \mathrm{C}$, and samples were irradiated with $659 \mathrm{~nm}$ light with the detector at $90^{\circ}$ from incident.

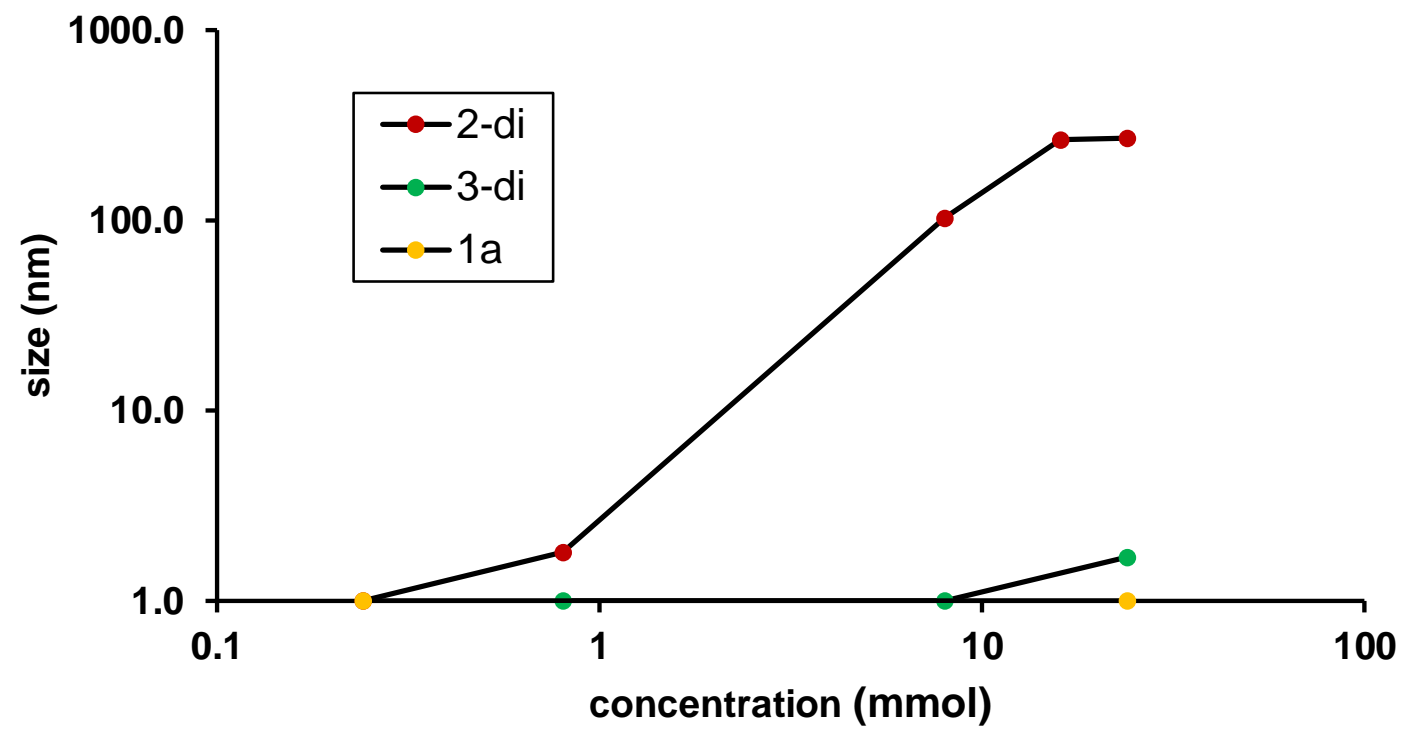

Figure S23. Particle size versus concentration for respective helicenes. Values were determined by taking the size values at maximum peak height in Figure S24.

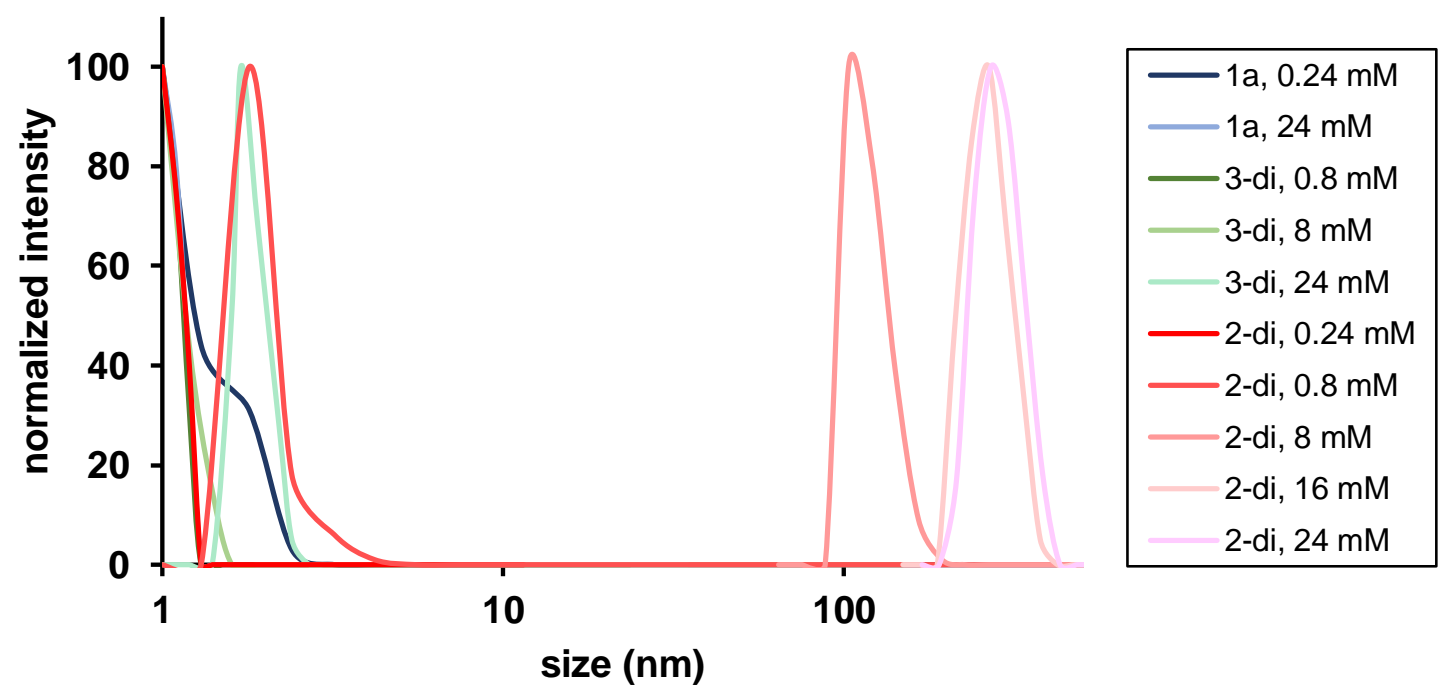

Figure S24. Raw multimodal distributions for helicenes 1a, 2-di, and 3-di, at various concentrations. 


\section{Estimation of helical pitch}

The pitch of a helix is defined as the height of one complete helical turn, measured parallel to the helical axis. ${ }^{9}$ The expanded helicenes reported herein have highly distorted structures and short lengths (approximately one helical turn), which necessitates an approach for pitch estimation that averages out local conformational effects. For this purpose, we previously used the HELFIT program, ${ }^{2,10}$ which employs a total least squares algorithm to estimate the parameters of a helix given a series of $3 D$ coordinates as the input. Here, the use of this program for expanded helicenes is described in more detail, using compound 1a as an example (Figure S25). The coordinates used herein are those defined by the centroids of the rings on the expanded helicene core. First, attempts were made to perform the least-squares fit using the coordinates for the entire set of centroids (i.e., all 11 of the centroids shown in Figure S25a). Unlike for the previouslyreported compounds, this gave an output that was physically unmeaningful. Thus, the centroids were broken up into two sets, inner and outer (red and blue, respectively, in Figure S25a), and each set independently gave an excellent fit. The values for the inner and outer centroids were then averaged.

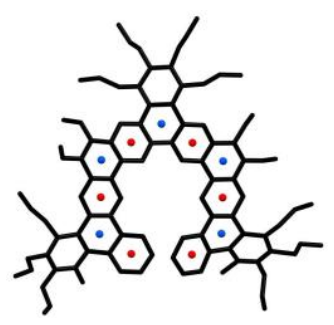

\begin{tabular}{|c|c|c|c|c|}
\cline { 2 - 5 } & \multicolumn{4}{|c|}{ HELFIT data for 1a } \\
\cline { 2 - 5 } & $\begin{array}{c}\text { All } \\
\text { centroids }\end{array}$ & $\begin{array}{c}\text { Inner } \\
\text { centroids }\end{array}$ & $\begin{array}{c}\text { Outer } \\
\text { centroids }\end{array}$ & $\begin{array}{c}\text { Average } \\
\text { of Inner \& } \\
\text { Outer }\end{array}$ \\
\hline Pitch $(\boldsymbol{A})$ & $\mathrm{N}^{\mathrm{ii}}$ & 3.24 & 4.17 & 3.71 \\
Data points (n) & 11 & 6 & 5 & 11 \\
Radius $(\mathbf{A})$ & $\mathrm{N}^{\mathrm{ii}}$ & 4.22 & 4.82 & 4.52 \\
RMSD/ $/(\mathbf{n}-\mathbf{1})^{\mathrm{i}}$ & 0.9 & 0.04 & 0.06 & N/A \\
\hline
\end{tabular}

Figure S25. Details of pitch calculation for compound 1a. The molecular structure on the left depicts the inner (red) and outer (blue) centroids used as input for HELFIT, and the compiled data on the right are the results of the HELFIT calculation for the noted centroids. (i) This value describes the regularity of the helix independent of length, and larger values indicate a larger error between the data points and the best fit helix. ${ }^{10}$ RMSD is the root-mean-square-distance of the data points to the best-fit helix and $n$ is the number of data points; (ii) these values were not physically meaningful were thus not included.

For the purposes of comparison, best-fit helices were estimated as described above from crystal structure data for all new compounds (1a, 2-mon, 2-di, 3-mon, 3-di), the DFT-optimized structures (2-mon-g, 3-mon-g, 3-di-g, Figure S27c), and all literature expanded helicenes for which crystal structures are available (S1, 1b-Dim, S2, Figure S26). ${ }^{2,11}$ The remaining values are summarized in Table S8.
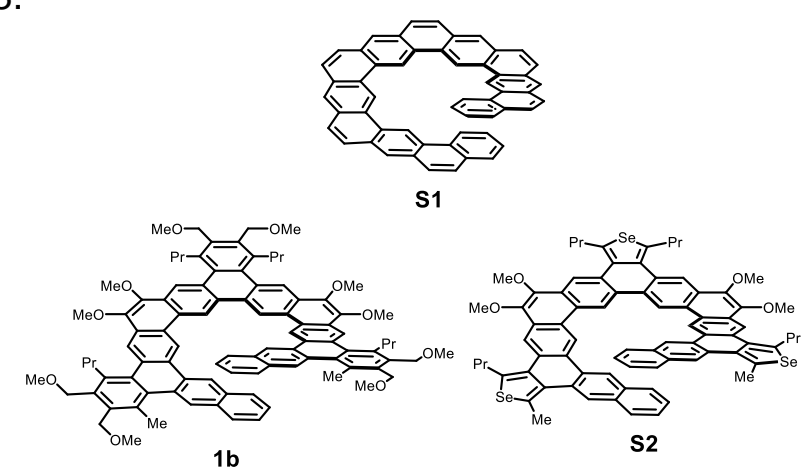

Figure S26. Structures of literature compounds used for comparison. 

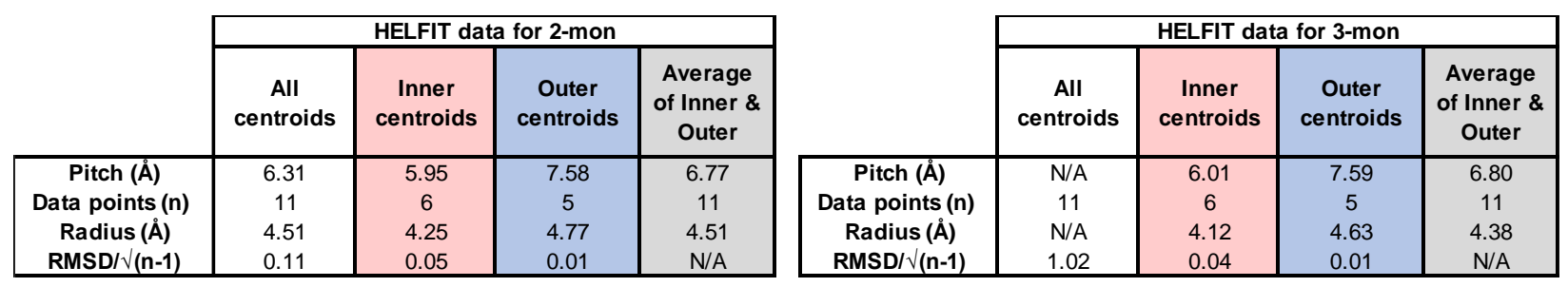

\begin{tabular}{|c|c|c|c|c|}
\cline { 2 - 5 } & \multicolumn{4}{|c|}{ HELFIT data for 2-di (1) ${ }^{*}$} \\
\cline { 2 - 5 } & $\begin{array}{c}\text { All } \\
\text { centroids }\end{array}$ & $\begin{array}{c}\text { Inner } \\
\text { centroids }\end{array}$ & $\begin{array}{c}\text { Outer } \\
\text { centroids }\end{array}$ & $\begin{array}{c}\text { Average } \\
\text { of Inner \& } \\
\text { Outer }\end{array}$ \\
\hline Pitch $(\AA)$ & N/A & 2.9 & 2.36 & 2.63 \\
Data points & 11 & 6 & 5 & 11 \\
Radius $(\AA)$ & N/A & 4.22 & 4.86 & 4.54 \\
RMSD/ $\sqrt{ }(\mathbf{n}-1)$ & 0.93 & 0.04 & 0.05 & N/A \\
\hline
\end{tabular}

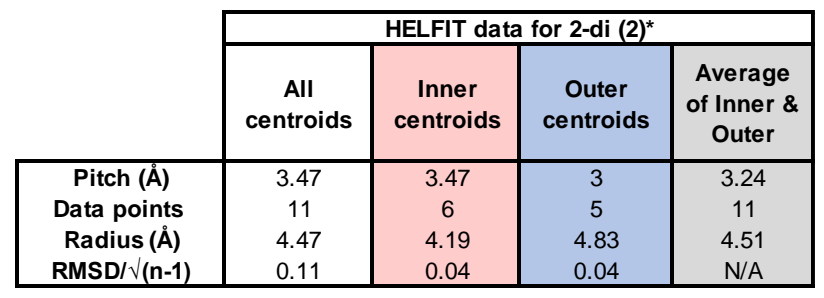

\begin{tabular}{|c|c|c|c|c|}
\hline & \multicolumn{4}{|c|}{ HELFIT data for 3-di } \\
\hline & $\begin{array}{c}\text { All } \\
\text { centroids }\end{array}$ & $\begin{array}{c}\text { Inner } \\
\text { centroids }\end{array}$ & $\begin{array}{l}\text { Outer } \\
\text { centroids }\end{array}$ & $\begin{array}{c}\text { Average } \\
\text { of Inner \& } \\
\text { Outer }\end{array}$ \\
\hline Pitch (Å) & 6.24 & 5.79 & 8.01 & 6.90 \\
\hline Data points & 11 & 6 & 5 & 11 \\
\hline Radius (Å) & 4.43 & 4.18 & 4.63 & 4.41 \\
\hline RMSD $/ \sqrt{ }(n-1)$ & 0.11 & 0.04 & 0.01 & $\mathrm{~N} / \mathrm{A}$ \\
\hline
\end{tabular}

\begin{tabular}{|c|c|c|c|c|}
\hline & \multicolumn{4}{|c|}{ HELFIT data for 2-mon-g } \\
\hline & $\begin{array}{c}\text { All } \\
\text { centroids }\end{array}$ & $\begin{array}{c}\text { Inner } \\
\text { centroids }\end{array}$ & $\begin{array}{c}\text { Outer } \\
\text { centroids }\end{array}$ & $\begin{array}{c}\text { Average } \\
\text { of Inner \& } \\
\text { Outer }\end{array}$ \\
\hline Pitch (£) & $\mathrm{N} / \mathrm{A}$ & 3.54 & 4.3 & 3.92 \\
\hline Data points & 11 & 6 & 5 & 11 \\
\hline Radius (Å) & $\mathrm{N} / \mathrm{A}$ & 4.34 & 4.98 & 4.66 \\
\hline $\operatorname{RMSD} / \sqrt{ }(\mathrm{n}-1)$ & 0.93 & 0.05 & 0.01 & $\mathrm{~N} / \mathrm{A}$ \\
\hline
\end{tabular}

\begin{tabular}{|c|c|c|c|c|}
\hline & \multicolumn{4}{|c|}{ HELFIT data for 3-mon-g } \\
\hline & $\begin{array}{c}\text { All } \\
\text { centroids }\end{array}$ & $\begin{array}{c}\text { Inner } \\
\text { centroids }\end{array}$ & $\begin{array}{c}\text { Outer } \\
\text { centroids }\end{array}$ & $\begin{array}{c}\text { Average } \\
\text { of Inner \& } \\
\text { Outer }\end{array}$ \\
\hline Pitch (Å) & $\mathrm{N} / \mathrm{A}$ & 3.49 & 4.33 & 3.91 \\
\hline Data points & 11 & 6 & 5 & 11 \\
\hline Radius (Å) & $\mathrm{N} / \mathrm{A}$ & 4.35 & 4.99 & 4.67 \\
\hline RMSD $/ \sqrt{ }(\mathrm{n}-1)$ & 0.93 & 0.05 & 0.01 & $\mathrm{~N} / \mathrm{A}$ \\
\hline
\end{tabular}

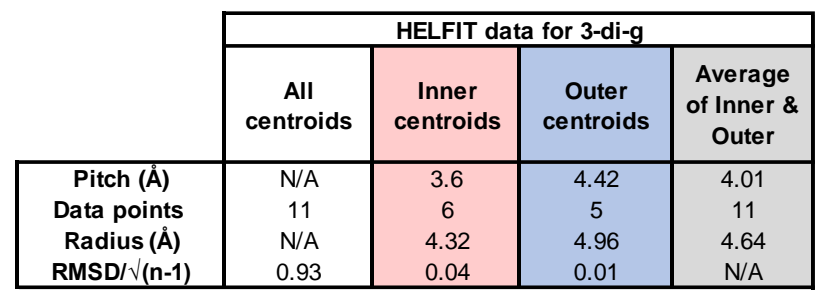

\begin{tabular}{|c|c|c|c|c|}
\hline & \multicolumn{4}{|c|}{ HELFIT data for S1 } \\
\hline & $\begin{array}{c}\text { All } \\
\text { centroids }\end{array}$ & $\begin{array}{c}\text { Inner } \\
\text { centroids }\end{array}$ & $\begin{array}{l}\text { Outer } \\
\text { centroids }\end{array}$ & $\begin{array}{c}\text { Average } \\
\text { of Inner \& } \\
\text { Outer }\end{array}$ \\
\hline Pitch $(\AA)$ & 3.23 & 2.81 & 3.39 & 3.10 \\
\hline Data points & 13 & 6 & 7 & 13 \\
\hline Radius (Å) & 4.63 & 4.29 & 4.91 & 4.60 \\
\hline $\operatorname{RMSD} / \sqrt{ }(\mathrm{n}-1)$ & 0.1 & 0.03 & 0.08 & N/A \\
\hline
\end{tabular}

\begin{tabular}{|c|c|c|c|c|}
\hline & \multicolumn{4}{|c|}{ HELFIT data for 1b-Dim } \\
\hline & $\begin{array}{c}\text { All } \\
\text { centroids }\end{array}$ & $\begin{array}{c}\text { Inner } \\
\text { centroids }\end{array}$ & $\begin{array}{c}\text { Outer } \\
\text { centroids }\end{array}$ & $\begin{array}{c}\text { Average } \\
\text { of Inner \& } \\
\text { Outer }\end{array}$ \\
\hline Pitch (Å) & 7.44 & 7.16 & 7.59 & 7.38 \\
\hline Data points & 13 & 6 & 7 & 13 \\
\hline Radius (A) & 4.5 & 4.18 & 4.78 & 4.48 \\
\hline RMSD $/ \sqrt{ }(n-1)$ & 0.09 & 0.04 & 0.03 & $\mathrm{~N} / \mathrm{A}$ \\
\hline
\end{tabular}

\begin{tabular}{|c|c|c|c|c|}
\hline & \multicolumn{4}{|c|}{ HELFIT data for S2 } \\
\hline & $\begin{array}{c}\text { All } \\
\text { centroids }\end{array}$ & $\begin{array}{c}\text { Inner } \\
\text { centroids }\end{array}$ & $\begin{array}{c}\text { Outer } \\
\text { centroids }\end{array}$ & $\begin{array}{c}\text { Average } \\
\text { of Inner \& } \\
\text { Outer }\end{array}$ \\
\hline Pitch $(\AA)$ & $\mathrm{N} / \mathrm{A}$ & 3.65 & 3.73 & 3.69 \\
\hline Data points & 13 & 7 & 6 & 13 \\
\hline Radius (Å) & $\mathrm{N} / \mathrm{A}$ & 4.39 & 5.05 & 4.72 \\
\hline RMSD $/ \sqrt{ }(\mathbf{n}-1)$ & 0.99 & 0.01 & 0.03 & N/A \\
\hline
\end{tabular}

Table S8. Compiled HELFIT data for relevant expanded helicenes. Data for $\mathbf{1 a}$ is presented in a model calculation in Figure 25. ${ }^{*}$ Compound 2-di crystallized with two symmetrically inequivalent geometries, pitches were calculated for both and found to be different. 


\section{DFT calculations}

Density functional theory (DFT) calculations were performed using the Gaussian 16 software package. ${ }^{12}$ Molecular mechanics calculations were used to generate starting geometries, and DFT optimization was carried out in $C_{1}$ symmetry in the gas-phase. Geometries were refined to meet standard convergence criteria, and confirmed to be local minima by normal mode frequency calculations. All DFT calculations were performed using the hybrid functional B3LYP, and the 6 $311 \mathrm{G}(2 \mathrm{~d}, \mathrm{p})$ basis set. This functional and basis set were chosen to maintain consistency with the only other published computational data on the structural flexibility of expanded helicenes. ${ }^{11}$ For all calculations, $n$-propyl groups were truncated to methyl groups, and (methoxy)methyl groups were removed (specific structures are described in the following paragraph). These groups are not expected to affect the strain energy significantly and were altered to allow for reasonable calculation times.

To approximate the energetic cost of the structural deformation observed in the crystal structure for 2-mon, 3-mon, and 3-di (Figure S27a), two calculations were performed on truncated versions of each compound. The first calculation was a standard, unconstrained optimization to simulate the gas-phase minimum energy structure (2-mon-g, 3-mon-g, and 3-di-g, Figure S27b). For the second calculation, two atoms on the terminal rings of the helicene were constrained to the distance observed in the crystal structure (2-mon-x, 3-mon-x, and 3-di-x, Figure S27c). Thermally corrected enthalpy values were then compared between the unconstrained and constrained optimized geometries to determine the enthalpic cost associated with the helical pitch elongation observed in the solid state.

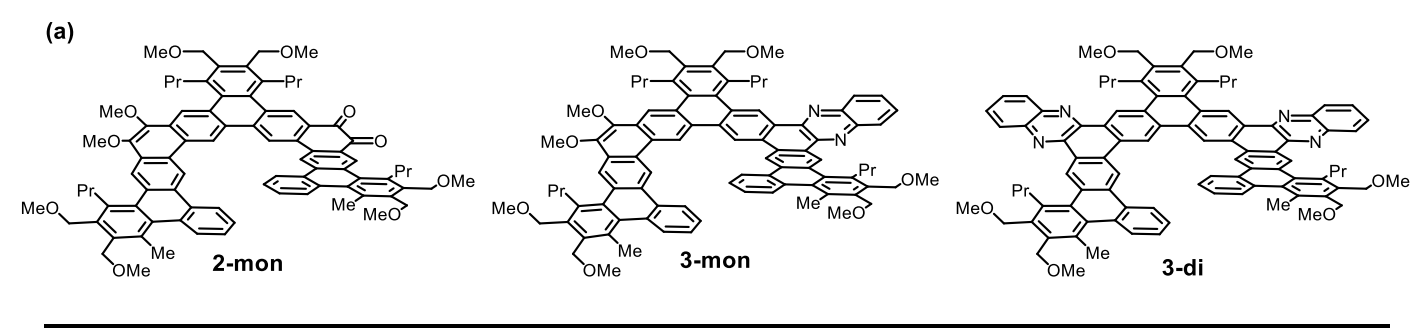

(b)
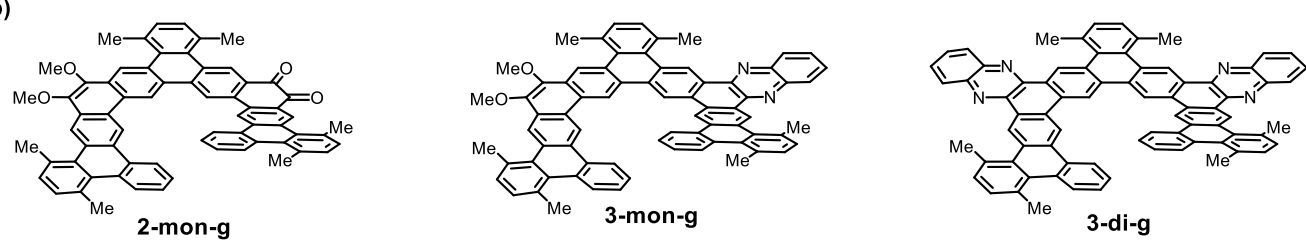

(c)
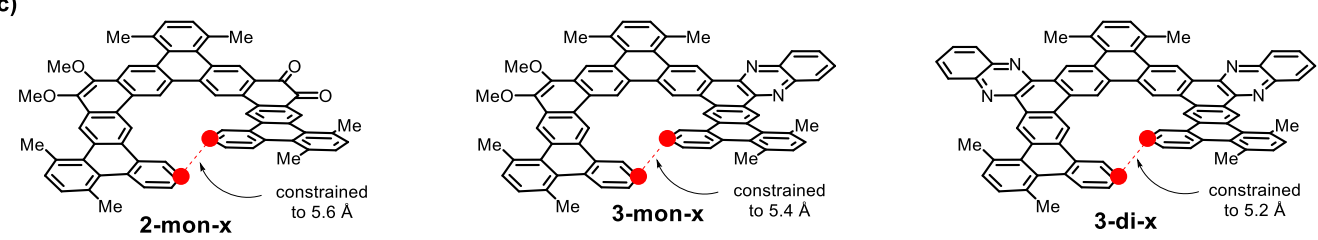

Figure S27. Details of the structures used for DFT calculations: (a) Full structures; (b) Truncated structures for unconstrained optimization; (c) Truncated structures for constrained optimization. 


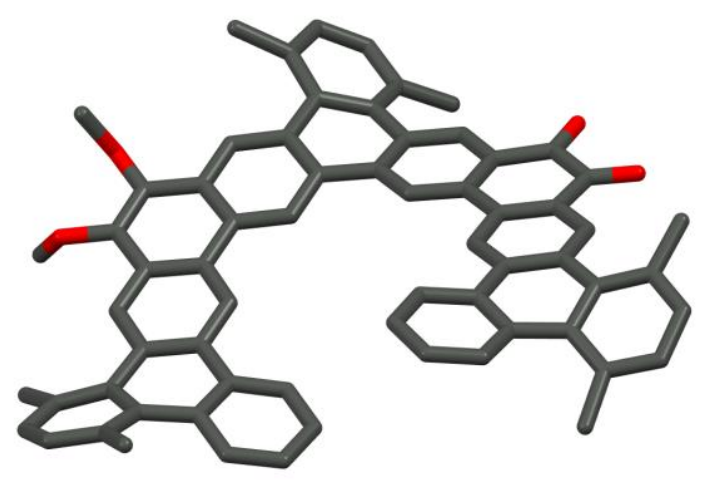

Figure S28. DFT calculated structure of 2-mon-g.

$\begin{array}{rrrrrrrr}\text { C } & 2.824 & 2.355 & -0.153 & \text { C } & 5.183 & 1.495 & -0.122 \\ \text { C } & 1.454 & 2.176 & -0.010 & \text { C } & 3.797 & 1.254 & 0.007 \\ \text { C } & 0.525 & 3.218 & -0.138 & \text { C } & 6.096 & 0.461 & 0.024 \\ \text { C } & 0.977 & 4.535 & -0.407 & \text { C } & 5.720 & -0.852 & 0.331 \\ \text { C } & 2.358 & 4.713 & -0.524 & \text { C } & 4.329 & -1.105 & 0.437 \\ \text { C } & 3.267 & 3.670 & -0.420 & \text { C } & 3.414 & -0.055 & 0.266 \\ \text { C } & -0.905 & 2.989 & 0.037 & \text { C } & 3.877 & -2.464 & 0.710 \\ \text { C } & -1.460 & 1.717 & -0.090 & \text { C } & 4.790 & -3.535 & 0.559 \\ \text { C } & -2.825 & 1.462 & 0.049 & \text { C } & 4.346 & -4.823 & 0.919 \\ \text { C } & -3.667 & 2.575 & 0.313 & \text { C } & 3.057 & -5.061 & 1.350 \\ \text { C } & -3.117 & 3.855 & 0.418 & \text { C } & 2.152 & -4.006 & 1.460 \\ \text { C } & -1.751 & 4.105 & 0.300 & \text { C } & 2.571 & -2.728 & 1.158 \\ \text { C } & -3.419 & 0.140 & -0.097 & \text { C } & -1.214 & 5.476 & 0.266 \\ \text { C } & -4.829 & 0.000 & -0.006 & \text { C } & 0.045 & 5.674 & -0.377 \\ \text { C } & -5.650 & 1.157 & 0.250 & \text { C } & -4.447 & -4.877 & -0.160 \\ \text { C } & -5.091 & 2.385 & 0.431 & \text { C } & -5.292 & -3.753 & -0.404 \\ \text { C } & -2.677 & -1.023 & -0.308 & \text { C } & 6.692 & -1.954 & 0.371 \\ \text { C } & -3.255 & -2.280 & -0.464 & \text { C } & 6.192 & -3.279 & 0.193 \\ \text { C } & -4.671 & -2.417 & -0.390 & \text { C } & 8.093 & -1.752 & 0.495 \\ \text { C } & -5.411 & -1.263 & -0.139 & \text { C } & 8.933 & -2.813 & 0.194 \\ \text { C } & -2.439 & -3.471 & -0.686 & \text { C } & 8.435 & -4.026 & -0.257 \\ \text { C } & -1.106 & -3.377 & -1.118 & \text { C } & 7.076 & -4.297 & -0.252 \\ \text { C } & -0.343 & -4.499 & -1.368 & \text { C } & -1.923 & 6.602 & 0.756 \\ \text { C } & -0.918 & -5.760 & -1.221 & \text { C } & -1.489 & 7.864 & 0.375 \\ \text { C } & -2.231 & -5.873 & -0.806 & \text { C } & -0.419 & 8.031 & -0.490 \\ \text { C } & -3.019 & -4.747 & -0.498 & \text { C } & 0.380 & 6.962 & -0.868 \\ \text { C } & 4.703 & 3.999 & -0.560 & \text { C } & -5.022 & -6.083 & 0.319 \\ \text { C } & 5.717 & 2.838 & -0.443 & \text { C } & -6.403 & -6.208 & 0.289\end{array}$




$\begin{array}{lrrrrrrr}\mathrm{C} & -7.206 & -5.205 & -0.230 & \mathrm{H} & 10.005 & -2.675 & 0.282 \\ \mathrm{C} & -6.687 & -3.963 & -0.565 & \mathrm{H} & 9.124 & -4.792 & -0.595 \\ \mathrm{C} & -3.081 & 6.547 & 1.730 & \mathrm{H} & -2.021 & 8.735 & 0.740 \\ \mathrm{C} & 1.505 & 7.259 & -1.837 & \mathrm{H} & -0.176 & 9.024 & -0.854 \\ \mathrm{C} & 8.751 & -0.487 & 1.003 & \mathrm{H} & -6.857 & -7.124 & 0.651 \\ \mathrm{C} & 6.649 & -5.640 & -0.810 & \mathrm{H} & -8.271 & -5.379 & -0.345 \\ \mathrm{C} & -7.658 & -2.959 & -1.150 & \mathrm{H} & -3.050 & 7.433 & 2.365 \\ \mathrm{C} & -4.249 & -7.225 & 0.946 & \mathrm{H} & -4.056 & 6.547 & 1.234 \\ \mathrm{O} & 5.112 & 5.122 & -0.760 & \mathrm{H} & -3.038 & 5.670 & 2.375 \\ \mathrm{O} & 6.895 & 3.062 & -0.610 & \mathrm{H} & 2.432 & 7.554 & -1.338 \\ \mathrm{O} & -5.865 & 3.496 & 0.677 & \mathrm{H} & 1.209 & 8.095 & -2.473 \\ \mathrm{C} & -6.465 & 3.535 & 1.980 & \mathrm{H} & 1.734 & 6.413 & -2.484 \\ \mathrm{O} & -7.008 & 0.965 & 0.354 & \mathrm{H} & 9.026 & 0.205 & 0.202 \\ \mathrm{C} & -7.783 & 1.535 & -0.711 & \mathrm{H} & 9.675 & -0.754 & 1.519 \\ \mathrm{H} & 1.078 & 1.200 & 0.258 & \mathrm{H} & 8.122 & 0.058 & 1.706 \\ \mathrm{H} & 2.789 & 5.690 & -0.657 & \mathrm{H} & 6.657 & -6.436 & -0.060 \\ \mathrm{H} & -0.806 & 0.900 & -0.356 & \mathrm{H} & 7.352 & -5.936 & -1.590 \\ \mathrm{H} & -3.814 & 4.664 & 0.532 & \mathrm{H} & 5.653 & -5.610 & -1.251 \\ \mathrm{H} & -1.600 & -0.953 & -0.316 & \mathrm{H} & -8.444 & -3.498 & -1.682 \\ \mathrm{H} & -6.472 & -1.315 & 0.020 & \mathrm{H} & -7.179 & -2.282 & -1.856 \\ \mathrm{H} & -0.671 & -2.404 & -1.302 & \mathrm{H} & -8.153 & -2.349 & -0.388 \\ \mathrm{H} & 0.682 & -4.396 & -1.703 & \mathrm{H} & -4.857 & -7.670 & 1.736 \\ \mathrm{H} & -0.351 & -6.652 & -1.459 & \mathrm{H} & -4.020 & -8.026 & 0.237 \\ \mathrm{H} & -2.668 & -6.857 & -0.772 & \mathrm{H} & -3.310 & -6.898 & 1.391 \\ \mathrm{H} & 7.123 & 0.724 & -0.154 & \mathrm{H} & -7.021 & 4.469 & 2.030 \\ \mathrm{H} & 2.363 & -0.292 & 0.308 & \mathrm{H} & -5.695 & 3.527 & 2.756 \\ \mathrm{H} & 5.038 & -5.648 & 0.913 & \mathrm{H} & -7.141 & 2.691 & 2.124 \\ \mathrm{H} & 2.761 & -6.065 & 1.628 & \mathrm{H} & -7.489 & 1.102 & -1.671 \\ \mathrm{H} & 1.141 & -4.180 & 1.805 & \mathrm{H} & -8.820 & 1.281 & -0.500 \\ \mathrm{H} & 1.882 & -1.909 & 1.312 & \mathrm{H} & -7.663 & 2.619 & -0.745\end{array}$

Electronic Energy: -2844.447121

Thermally Corrected Gibbs Free Energy: -2843.613809

Thermally Corrected Enthalpy: -2843.46627

Table S9. Cartesian coordinates of calculated 2-mon-g. 


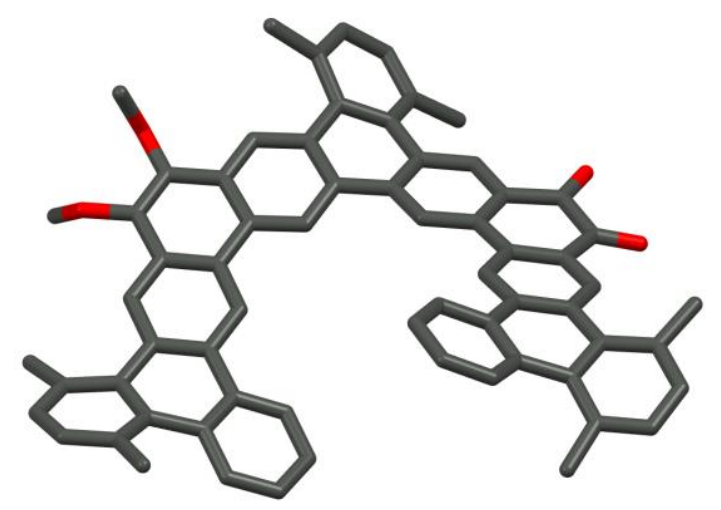

Figure S29. DFT calculated structure of 2-mon-x.

\begin{tabular}{|c|c|c|c|c|c|c|c|}
\hline C & -0.546 & -4.351 & -2.120 & C & -0.886 & 2.968 & 0.035 \\
\hline$C$ & -1.105 & -5.623 & -2.013 & $\mathrm{C}$ & -1.453 & 1.704 & -0.108 \\
\hline C & -2.356 & -5.780 & -1.448 & C & 0.061 & 5.644 & -0.412 \\
\hline$C$ & -3.093 & -4.689 & -0.948 & $\mathrm{C}$ & -1.166 & 5.452 & 0.296 \\
\hline C & -2.533 & -3.399 & -1.092 & C & 0.377 & 6.931 & -0.918 \\
\hline C & -1.264 & -3.259 & -1.677 & C & -1.420 & 7.842 & 0.421 \\
\hline$C$ & -4.469 & -4.853 & -0.451 & $\mathrm{C}$ & -0.395 & 8.004 & -0.498 \\
\hline$C$ & -4.981 & -6.096 & 0.003 & $\mathrm{C}$ & -1.842 & 6.583 & 0.821 \\
\hline$C$ & -6.355 & -6.232 & 0.132 & C & -2.951 & 6.532 & 1.850 \\
\hline$C$ & -7.215 & -5.201 & -0.212 & $\mathrm{C}$ & 1.453 & 7.225 & -1.941 \\
\hline C & -6.741 & -3.933 & -0.517 & $C$ & 0.986 & 4.500 & -0.484 \\
\hline C & -5.337 & -3.721 & -0.510 & C & 0.538 & 3.190 & -0.179 \\
\hline$C$ & -4.135 & -7.273 & 0.444 & $\mathrm{C}$ & 2.828 & 2.315 & -0.241 \\
\hline$C$ & -7.779 & -2.901 & -0.906 & $\mathrm{C}$ & 1.463 & 2.146 & -0.048 \\
\hline $\mathrm{C}$ & -4.717 & -2.384 & -0.470 & $\mathrm{C}$ & 4.688 & 3.905 & -0.870 \\
\hline$C$ & -3.314 & -2.237 & -0.677 & $\mathrm{C}$ & 2.360 & 4.660 & -0.686 \\
\hline C & -2.715 & -0.997 & -0.475 & C & 3.264 & 3.610 & -0.598 \\
\hline C & -4.826 & 0.004 & 0.081 & C & 3.807 & 1.225 & -0.039 \\
\hline C & -3.427 & 0.147 & -0.111 & $C$ & 5.170 & 1.419 & -0.351 \\
\hline$C$ & -5.424 & -1.249 & -0.077 & $\mathrm{C}$ & 5.675 & 2.715 & -0.859 \\
\hline$C$ & -5.618 & 1.147 & 0.457 & $\mathrm{C}$ & 3.451 & -0.023 & 0.451 \\
\hline C & -5.043 & 2.368 & 0.642 & $\mathrm{C}$ & 5.741 & -0.865 & 0.327 \\
\hline C & -3.631 & 2.560 & 0.433 & C & 4.375 & -1.058 & 0.660 \\
\hline$C$ & -6.284 & 3.464 & 2.335 & $\mathrm{C}$ & 6.693 & -1.985 & 0.381 \\
\hline C & -2.814 & 1.454 & 0.075 & $\mathrm{C}$ & 6.085 & 0.389 & -0.193 \\
\hline C & -7.818 & 1.560 & -0.322 & $\mathrm{C}$ & 8.861 & -2.951 & -0.020 \\
\hline C & -3.070 & 3.836 & 0.541 & C & 8.099 & -1.825 & 0.249 \\
\hline C & -1.712 & 4.085 & 0.351 & $\mathrm{C}$ & 8.279 & -4.197 & -0.198 \\
\hline
\end{tabular}




\begin{tabular}{|c|c|c|c|c|c|c|c|}
\hline$C$ & 8.856 & -0.528 & 0.448 & $\mathrm{H}$ & -6.947 & 2.614 & 2.507 \\
\hline$C$ & 6.152 & -3.303 & 0.486 & $\mathrm{H}$ & -8.837 & 1.299 & -0.039 \\
\hline$C$ & 6.935 & -4.409 & 0.065 & $\mathrm{H}$ & -7.602 & 1.160 & -1.316 \\
\hline$C$ & 6.399 & -5.801 & -0.204 & $\mathrm{H}$ & -7.699 & 2.644 & -0.326 \\
\hline$C$ & 2.739 & -2.479 & 1.904 & $\mathrm{H}$ & -3.757 & 4.642 & 0.717 \\
\hline$C$ & 2.370 & -3.674 & 2.484 & $\mathrm{H}$ & -0.813 & 0.893 & -0.422 \\
\hline$C$ & 3.236 & -4.764 & 2.408 & $\mathrm{H}$ & -1.929 & 8.716 & 0.812 \\
\hline$C$ & 4.439 & -4.646 & 1.741 & $\mathrm{H}$ & -0.165 & 8.995 & -0.872 \\
\hline$C$ & 4.826 & -3.450 & 1.104 & $\mathrm{H}$ & -2.879 & 5.657 & 2.496 \\
\hline$C$ & 3.957 & -2.339 & 1.216 & $\mathrm{H}$ & -3.949 & 6.534 & 1.402 \\
\hline 0 & -6.965 & 0.952 & 0.660 & $\mathrm{H}$ & -2.887 & 7.421 & 2.481 \\
\hline 0 & -5.795 & 3.469 & 0.986 & $\mathrm{H}$ & 1.637 & 6.383 & -2.607 \\
\hline 0 & 5.104 & 5.016 & -1.114 & $\mathrm{H}$ & 2.409 & 7.504 & -1.488 \\
\hline 0 & 6.814 & 2.885 & -1.235 & $\mathrm{H}$ & 1.136 & 8.071 & -2.553 \\
\hline $\mathrm{H}$ & 0.427 & -4.214 & -2.576 & $\mathrm{H}$ & 1.089 & 1.180 & 0.256 \\
\hline $\mathrm{H}$ & -0.577 & -6.488 & -2.397 & $\mathrm{H}$ & 2.792 & 5.625 & -0.883 \\
\hline $\mathrm{H}$ & -2.791 & -6.765 & -1.445 & $\mathrm{H}$ & 2.414 & -0.223 & 0.671 \\
\hline $\mathrm{H}$ & -0.851 & -2.271 & -1.827 & $\mathrm{H}$ & 7.084 & 0.606 & -0.525 \\
\hline $\mathrm{H}$ & -6.762 & -7.175 & 0.478 & $\mathrm{H}$ & 9.935 & -2.844 & -0.127 \\
\hline $\mathrm{H}$ & -8.286 & -5.375 & -0.210 & $\mathrm{H}$ & 8.891 & -5.031 & -0.523 \\
\hline $\mathrm{H}$ & -3.146 & -6.970 & 0.786 & $\mathrm{H}$ & 8.374 & 0.131 & 1.170 \\
\hline $\mathrm{H}$ & -4.000 & -8.021 & -0.343 & $\mathrm{H}$ & 8.990 & 0.036 & -0.478 \\
\hline $\mathrm{H}$ & -4.637 & -7.777 & 1.273 & $\mathrm{H}$ & 9.854 & -0.759 & 0.824 \\
\hline $\mathrm{H}$ & -8.629 & -3.412 & -1.363 & $\mathrm{H}$ & 6.952 & -6.236 & -1.038 \\
\hline $\mathrm{H}$ & -7.396 & -2.179 & -1.627 & $\mathrm{H}$ & 5.343 & -5.796 & -0.470 \\
\hline $\mathrm{H}$ & -8.169 & -2.342 & -0.050 & $\mathrm{H}$ & 6.525 & -6.479 & 0.645 \\
\hline $\mathrm{H}$ & -1.643 & -0.927 & -0.583 & $\mathrm{H}$ & 2.092 & -1.622 & 2.026 \\
\hline $\mathrm{H}$ & -6.467 & -1.309 & 0.169 & $\mathrm{H}$ & 1.431 & -3.753 & 3.017 \\
\hline $\mathrm{H}$ & -6.834 & 4.395 & 2.462 & $\mathrm{H}$ & 2.983 & -5.698 & 2.896 \\
\hline $\mathrm{H}$ & -5.452 & 3.432 & 3.044 & $\mathrm{H}$ & 5.115 & -5.483 & 1.761 \\
\hline
\end{tabular}

Electronic Energy: -2844.445891

Thermally Corrected Gibbs Free Energy: -2843.631993

Thermally Corrected Enthalpy: -2843.47129

Table S10. Cartesian coordinates of calculated 2-mon-x. 


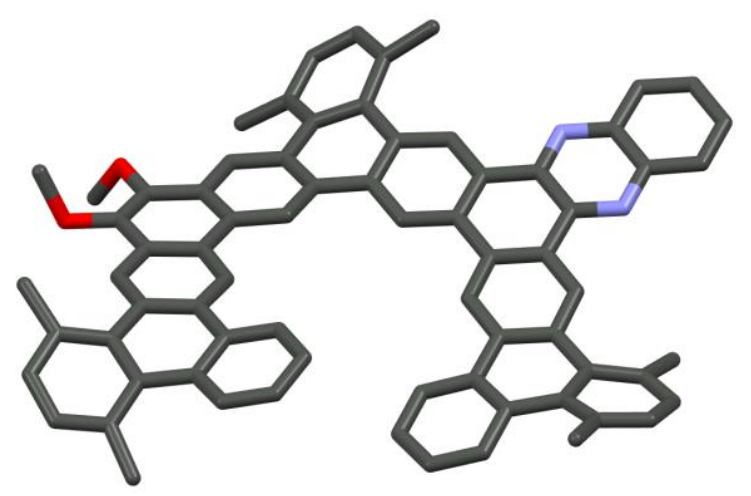

Figure S30. DFT calculated structure of 3-mon-g.

\begin{tabular}{|c|c|c|c|c|c|c|c|}
\hline C & -1.514 & -1.890 & -0.025 & C & 2.120 & 3.850 & 0.758 \\
\hline C & -2.889 & -2.108 & 0.088 & C & 2.643 & 5.156 & 0.605 \\
\hline C & -3.312 & -3.435 & 0.365 & C & 1.790 & 6.238 & 0.904 \\
\hline C & -2.364 & -4.452 & 0.504 & $C$ & 0.473 & 6.054 & 1.277 \\
\hline C & -0.993 & -4.228 & 0.412 & C & -0.045 & 4.765 & 1.388 \\
\hline C & -0.566 & -2.897 & 0.139 & C & 0.780 & 3.685 & 1.147 \\
\hline C & -3.890 & -1.067 & -0.098 & C & -4.171 & 2.652 & -0.759 \\
\hline C & -5.266 & -1.409 & -0.029 & C & -2.874 & 3.006 & -1.167 \\
\hline C & -5.655 & -2.769 & 0.244 & C & -2.528 & 4.315 & -1.433 \\
\hline C & -4.718 & -3.734 & 0.459 & C & -3.498 & 5.310 & -1.327 \\
\hline C & -3.579 & 0.274 & -0.325 & C & -4.782 & 4.981 & -0.937 \\
\hline C & -4.543 & 1.260 & -0.521 & C & -5.151 & 3.661 & -0.613 \\
\hline C & -5.923 & 0.913 & -0.470 & C & -6.546 & 3.308 & -0.299 \\
\hline C & -6.237 & -0.419 & -0.201 & C & -6.958 & 1.960 & -0.527 \\
\hline C & 0.863 & -2.633 & -0.005 & C & -0.024 & -5.339 & 0.409 \\
\hline$C$ & 1.741 & -3.723 & -0.243 & C & 1.241 & -5.109 & -0.212 \\
\hline$C$ & 3.104 & -3.436 & -0.326 & C & -0.322 & -6.634 & 0.904 \\
\hline C & 3.617 & -2.147 & -0.221 & C & 0.515 & -7.681 & 0.547 \\
\hline C & 2.746 & -1.058 & 0.013 & C & 1.592 & -7.487 & -0.304 \\
\hline C & 1.386 & -1.345 & 0.125 & C & 1.993 & -6.215 & -0.684 \\
\hline C & 5.062 & -1.933 & -0.317 & C & -7.504 & 4.258 & 0.141 \\
\hline C & 5.596 & -0.608 & -0.158 & C & -8.845 & 3.909 & 0.088 \\
\hline$C$ & 4.688 & 0.511 & 0.100 & C & -9.252 & 2.685 & -0.417 \\
\hline$C$ & 3.292 & 0.297 & 0.171 & C & -8.338 & 1.685 & -0.713 \\
\hline C & 5.202 & 1.795 & 0.251 & C & 4.961 & 4.273 & 0.552 \\
\hline C & 4.404 & 2.912 & 0.504 & C & 4.070 & 5.361 & 0.308 \\
\hline C & 2.999 & 2.705 & 0.547 & C & 6.342 & 4.542 & 0.749 \\
\hline C & 2.489 & 1.417 & 0.373 & C & 6.809 & 5.813 & 0.450 \\
\hline
\end{tabular}




\begin{tabular}{|c|c|c|c|c|c|c|c|}
\hline$C$ & 5.972 & 6.789 & -0.068 & $\mathrm{H}$ & -5.525 & 5.760 & -0.935 \\
\hline$C$ & 4.600 & 6.602 & -0.134 & $\mathrm{H}$ & 0.298 & -8.678 & 0.916 \\
\hline 0 & -5.079 & -5.037 & 0.719 & $\mathrm{H}$ & 2.158 & -8.344 & -0.654 \\
\hline$C$ & -5.652 & -5.253 & 2.017 & $\mathrm{H}$ & -9.590 & 4.625 & 0.421 \\
\hline 0 & -7.000 & -3.046 & 0.327 & $\mathrm{H}$ & -10.310 & 2.487 & -0.552 \\
\hline$C$ & -7.517 & -3.854 & -0.740 & $\mathrm{H}$ & 7.862 & 6.034 & 0.593 \\
\hline$C$ & -1.448 & -6.960 & 1.863 & $\mathrm{H}$ & 6.392 & 7.732 & -0.401 \\
\hline$C$ & 3.165 & -6.124 & -1.638 & $\mathrm{H}$ & -6.575 & -4.683 & 2.136 \\
\hline$C$ & 7.344 & 3.570 & 1.336 & $\mathrm{H}$ & -5.863 & -6.319 & 2.082 \\
\hline$C$ & 3.790 & 7.721 & -0.758 & $\mathrm{H}$ & -4.942 & -4.973 & 2.800 \\
\hline C & -7.175 & 5.605 & 0.751 & $\mathrm{H}$ & -7.371 & -3.357 & -1.703 \\
\hline C & -8.901 & 0.401 & -1.287 & $\mathrm{H}$ & -8.583 & -3.965 & -0.547 \\
\hline $\mathrm{N}$ & 5.853 & -2.965 & -0.548 & $\mathrm{H}$ & -7.038 & -4.834 & -0.755 \\
\hline $\mathrm{C}$ & 7.181 & -2.744 & -0.631 & $\mathrm{H}$ & -2.357 & -7.295 & 1.355 \\
\hline C & 7.711 & -1.428 & -0.474 & $\mathrm{H}$ & -1.714 & -6.113 & 2.494 \\
\hline $\mathrm{N}$ & 6.894 & -0.380 & -0.237 & $\mathrm{H}$ & -1.131 & -7.778 & 2.514 \\
\hline C & 8.066 & -3.822 & -0.878 & $\mathrm{H}$ & 4.131 & -6.094 & -1.127 \\
\hline C & 9.414 & -3.597 & -0.964 & $\mathrm{H}$ & 3.109 & -5.249 & -2.284 \\
\hline $\mathrm{C}$ & 9.940 & -2.291 & -0.808 & $\mathrm{H}$ & 3.171 & -7.011 & -2.275 \\
\hline$C$ & 9.111 & -1.228 & -0.568 & $\mathrm{H}$ & 7.877 & 2.993 & 0.575 \\
\hline $\mathrm{H}$ & -1.167 & -0.905 & -0.299 & $\mathrm{H}$ & 6.882 & 2.860 & 2.021 \\
\hline $\mathrm{H}$ & -2.750 & -5.447 & 0.625 & $\mathrm{H}$ & 8.096 & 4.133 & 1.892 \\
\hline $\mathrm{H}$ & -2.541 & 0.571 & -0.317 & $\mathrm{H}$ & 3.502 & 8.493 & -0.039 \\
\hline $\mathrm{H}$ & -7.256 & -0.724 & -0.058 & $\mathrm{H}$ & 2.882 & 7.360 & -1.239 \\
\hline $\mathrm{H}$ & 3.829 & -4.223 & -0.429 & $\mathrm{H}$ & 4.399 & 8.212 & -1.519 \\
\hline $\mathrm{H}$ & 0.704 & -0.546 & 0.372 & $\mathrm{H}$ & -6.191 & 5.623 & 1.217 \\
\hline $\mathrm{H}$ & 6.264 & 1.892 & 0.119 & $\mathrm{H}$ & -7.915 & 5.834 & 1.521 \\
\hline $\mathrm{H}$ & 1.417 & 1.293 & 0.363 & $\mathrm{H}$ & -7.214 & 6.424 & 0.026 \\
\hline $\mathrm{H}$ & 2.178 & 7.242 & 0.896 & $\mathrm{H}$ & -9.810 & 0.634 & -1.845 \\
\hline $\mathrm{H}$ & -0.144 & 6.913 & 1.508 & $\mathrm{H}$ & -9.178 & -0.326 & -0.518 \\
\hline $\mathrm{H}$ & -1.073 & 4.607 & 1.688 & $\mathrm{H}$ & -8.205 & -0.087 & -1.969 \\
\hline $\mathrm{H}$ & 0.387 & 2.690 & 1.302 & $\mathrm{H}$ & 7.641 & -4.812 & -0.994 \\
\hline $\mathrm{H}$ & -2.132 & 2.234 & -1.318 & $\mathrm{H}$ & 10.090 & -4.423 & -1.153 \\
\hline $\mathrm{H}$ & -1.521 & 4.558 & -1.747 & $\mathrm{H}$ & 11.010 & -2.138 & -0.880 \\
\hline $\mathrm{H}$ & -3.259 & 6.337 & -1.578 & $\mathrm{H}$ & 9.491 & -0.222 & -0.446 \\
\hline
\end{tabular}

Electronic Energy: -3034.629832

Thermally Corrected Gibbs Free Energy: -3033.716155

Thermally Corrected Enthalpy: -3033.561714

Table S11. Cartesian coordinates of calculated 3-mon-g. 


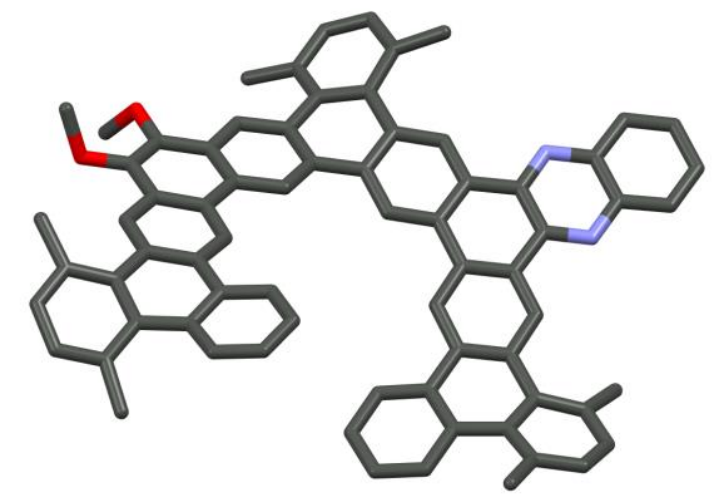

Figure S31. DFT calculated structure of calculated 3-mon-x.

\begin{tabular}{|c|c|c|c|c|c|c|c|}
\hline C & 3.678 & 7.735 & -0.227 & C & 7.106 & -2.750 & -0.948 \\
\hline C & 4.566 & 6.588 & 0.212 & C & 7.968 & -3.825 & -1.280 \\
\hline C & 5.935 & 6.785 & 0.112 & C & 9.304 & -3.598 & -1.471 \\
\hline C & 6.833 & 5.786 & 0.454 & C & 9.842 & -2.294 & -1.341 \\
\hline 0 & -7.014 & -2.929 & 0.592 & C & 3.059 & -3.443 & -0.366 \\
\hline C & 6.410 & 4.494 & 0.730 & C & 1.699 & -3.723 & -0.223 \\
\hline 0 & -5.105 & -4.922 & 1.023 & C & 0.839 & -2.624 & 0.035 \\
\hline C & 5.017 & 4.222 & 0.693 & C & 1.374 & -1.344 & 0.172 \\
\hline C & 4.101 & 5.316 & 0.635 & C & 1.186 & -5.103 & -0.156 \\
\hline C & 7.484 & 3.500 & 1.119 & C & 1.911 & -6.226 & -0.629 \\
\hline C & 2.723 & 5.081 & 1.099 & C & 3.055 & -6.166 & -1.620 \\
\hline C & 1.924 & 6.131 & 1.594 & C & 1.512 & -7.487 & -0.210 \\
\hline C & 0.670 & 5.908 & 2.127 & $\mathrm{~N}$ & 6.855 & -0.393 & -0.493 \\
\hline C & 0.167 & 4.611 & 2.204 & $\mathrm{~N}$ & 5.788 & -2.973 & -0.765 \\
\hline C & 0.943 & 3.559 & 1.764 & C & -6.990 & 5.766 & 0.181 \\
\hline$C$ & 2.220 & 3.765 & 1.213 & C & -7.395 & 4.368 & -0.241 \\
\hline$C$ & 3.063 & 2.647 & 0.805 & C & -8.742 & 4.054 & -0.150 \\
\hline C & 4.456 & 2.862 & 0.624 & C & -9.211 & 2.792 & -0.479 \\
\hline C & 5.221 & 1.761 & 0.232 & C & -8.342 & 1.740 & -0.730 \\
\hline C & 4.688 & 0.487 & 0.058 & C & -6.946 & 1.994 & -0.682 \\
\hline C & 3.301 & 0.273 & 0.230 & C & -6.494 & 3.348 & -0.640 \\
\hline C & 2.529 & 1.375 & 0.588 & C & -8.977 & 0.418 & -1.110 \\
\hline C & 3.583 & -2.156 & -0.271 & C & -5.122 & 3.627 & -1.098 \\
\hline$C$ & 5.019 & -1.944 & -0.456 & C & -4.762 & 4.886 & -1.616 \\
\hline C & 5.567 & -0.623 & -0.313 & C & -3.509 & 5.131 & -2.144 \\
\hline C & 2.734 & -1.068 & 0.032 & C & -2.564 & 4.108 & -2.192 \\
\hline C & 9.036 & -1.235 & -1.020 & C & -2.904 & 2.853 & -1.729 \\
\hline C & 7.649 & -1.436 & -0.815 & C & -4.170 & 2.586 & -1.182 \\
\hline
\end{tabular}




$\begin{array}{rrrrrrrr}\mathrm{C} & -4.547 & 1.244 & -0.749 & \mathrm{H} & 7.535 & -4.813 & -1.375 \\ \mathrm{C} & -5.928 & 0.932 & -0.583 & \mathrm{H} & 9.963 & -4.420 & -1.725 \\ \mathrm{C} & -6.243 & -0.362 & -0.173 & \mathrm{H} & 10.903 & -2.139 & -1.498 \\ \mathrm{C} & -5.277 & -1.350 & 0.041 & \mathrm{H} & 3.775 & -4.230 & -0.513 \\ \mathrm{C} & -3.901 & -1.035 & -0.110 & \mathrm{H} & 0.701 & -0.544 & 0.443 \\ \mathrm{C} & -3.586 & 0.270 & -0.491 & \mathrm{H} & 2.983 & -5.307 & -2.287 \\ \mathrm{C} & -3.332 & -3.371 & 0.502 & \mathrm{H} & 3.038 & -7.069 & -2.232 \\ \mathrm{C} & -2.905 & -2.068 & 0.134 & \mathrm{H} & 4.036 & -6.127 & -1.138 \\ \mathrm{C} & -1.529 & -1.866 & -0.003 & \mathrm{H} & 2.059 & -8.355 & -0.561 \\ \mathrm{C} & -0.590 & -2.874 & 0.196 & \mathrm{H} & -7.656 & 6.099 & 0.980 \\ \mathrm{C} & -1.022 & -4.191 & 0.519 & \mathrm{H} & -7.077 & 6.498 & -0.627 \\ \mathrm{C} & -2.392 & -4.394 & 0.662 & \mathrm{H} & -5.969 & 5.808 & 0.557 \\ \mathrm{C} & -4.737 & -3.644 & 0.667 & \mathrm{H} & -9.445 & 4.822 & 0.155 \\ \mathrm{C} & -5.670 & -2.680 & 0.431 & \mathrm{H} & -10.279 & 2.610 & -0.508 \\ \mathrm{C} & -7.590 & -3.792 & -0.400 & \mathrm{H} & -9.932 & 0.615 & -1.602 \\ \mathrm{C} & -5.614 & -5.049 & 2.358 & \mathrm{H} & -9.192 & -0.217 & -0.246 \\ \mathrm{C} & -0.061 & -5.310 & 0.512 & \mathrm{H} & -8.358 & -0.157 & -1.798 \\ \mathrm{C} & -0.354 & -6.592 & 1.041 & \mathrm{H} & -5.493 & 5.676 & -1.659 \\ \mathrm{C} & -1.453 & -6.889 & 2.040 & \mathrm{H} & -3.277 & 6.110 & -2.544 \\ \mathrm{C} & 0.462 & -7.654 & 0.678 & \mathrm{H} & -1.583 & 4.284 & -2.616 \\ \mathrm{H} & 4.177 & 8.278 & -1.033 & \mathrm{H} & -2.188 & 2.050 & -1.834 \\ \mathrm{H} & 3.491 & 8.460 & 0.571 & \mathrm{H} & -7.257 & -0.639 & 0.044 \\ \mathrm{H} & 2.713 & 7.396 & -0.600 & \mathrm{H} & -2.546 & 0.549 & -0.570 \\ \mathrm{H} & 6.307 & 7.751 & -0.211 & \mathrm{H} & -1.174 & -0.897 & -0.322 \\ \mathrm{H} & 7.894 & 6.007 & 0.475 & \mathrm{H} & -2.788 & -5.376 & 0.842 \\ \mathrm{H} & 8.307 & 4.039 & 1.594 & \mathrm{H} & -8.648 & -3.872 & -0.154 \\ \mathrm{H} & 7.906 & 2.967 & 0.262 & \mathrm{H} & -7.126 & -4.779 & -0.376 \\ \mathrm{H} & 7.124 & 2.752 & 1.824 & \mathrm{H} & -7.480 & -3.356 & -1.397 \\ \mathrm{H} & 2.313 & 7.135 & 1.615 & \mathrm{H} & -5.837 & -6.106 & 2.499 \\ \mathrm{H} & 0.093 & 6.742 & 2.509 & \mathrm{H} & -6.522 & -4.457 & 2.487 \\ \mathrm{H} & -0.811 & 4.423 & 2.632 & \mathrm{H} & -4.862 & -4.735 & 3.087 \\ \mathrm{H} & 0.572 & 2.552 & 1.890 & \mathrm{H} & -1.688 & -6.029 & 2.665 \\ \mathrm{H} & 6.267 & 1.862 & 0.012 & \mathrm{H} & -1.126 & -7.701 & 2.693 \\ \mathrm{H} & 1.461 & 1.253 & 0.683 & \mathrm{H} & -2.382 & -7.220 & 1.565 \\ \mathrm{H} & 9.425 & -0.229 & -0.915 & \mathrm{H} & 0.248 & -8.641 & 1.073 \\ & & & & & & \end{array}$

Electronic Energy: -3034.628702

Thermally Corrected Gibbs Free Energy: -3033.732856

Thermally Corrected Enthalpy: -3033.566814

Table S12. Cartesian coordinates of calculated 3-mon-x. 


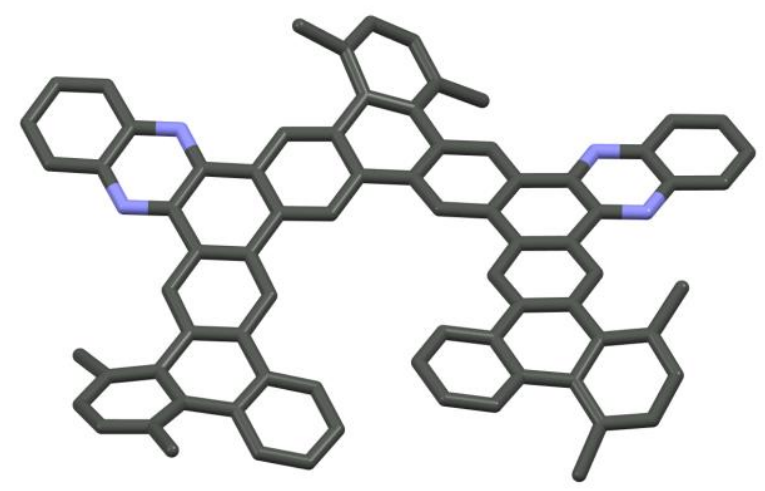

Figure S32. DFT calculated structure of 3-di-g.

\begin{tabular}{|c|c|c|c|c|c|c|c|}
\hline C & -2.865 & -1.374 & 0.007 & C & -3.647 & -0.144 & 0.195 \\
\hline C & -1.474 & -1.409 & 0.097 & C & -5.797 & 0.979 & 0.310 \\
\hline C & -0.727 & -2.577 & -0.060 & C & -5.214 & 2.219 & 0.577 \\
\hline C & -1.394 & -3.808 & -0.300 & C & -3.795 & 2.270 & 0.614 \\
\hline C & -2.787 & -3.773 & -0.365 & C & -3.060 & 1.099 & 0.414 \\
\hline C & -3.525 & -2.600 & -0.234 & C & -3.137 & 3.552 & 0.844 \\
\hline C & 0.727 & -2.577 & 0.060 & C & -3.888 & 4.744 & 0.712 \\
\hline C & 1.474 & -1.409 & -0.097 & C & -3.245 & 5.957 & 1.029 \\
\hline C & 2.865 & -1.374 & -0.007 & C & -1.916 & 6.008 & 1.400 \\
\hline C & 3.525 & -2.600 & 0.234 & C & -1.173 & 4.833 & 1.491 \\
\hline C & 2.787 & -3.773 & 0.365 & C & -1.788 & 3.626 & 1.231 \\
\hline C & 1.394 & -3.808 & 0.301 & C & 0.649 & -5.079 & 0.297 \\
\hline C & 3.647 & -0.144 & -0.195 & C & -0.649 & -5.079 & -0.297 \\
\hline C & 5.059 & -0.187 & -0.136 & C & 5.330 & 4.691 & -0.419 \\
\hline C & 5.750 & -1.449 & 0.132 & C & 6.009 & 3.456 & -0.648 \\
\hline$C$ & 4.986 & -2.652 & 0.313 & C & -6.009 & 3.456 & 0.647 \\
\hline C & 3.059 & 1.100 & -0.414 & C & -5.330 & 4.691 & 0.419 \\
\hline C & 3.794 & 2.270 & -0.613 & C & -7.415 & 3.468 & 0.850 \\
\hline C & 5.214 & 2.220 & -0.577 & C & -8.106 & 4.637 & 0.571 \\
\hline C & 5.797 & 0.979 & -0.310 & C & -7.460 & 5.756 & 0.067 \\
\hline C & 3.137 & 3.552 & -0.844 & C & -6.077 & 5.822 & -0.003 \\
\hline C & 1.788 & 3.626 & -1.230 & C & 1.194 & -6.300 & 0.769 \\
\hline C & 1.173 & 4.833 & -1.490 & C & 0.557 & -7.480 & 0.413 \\
\hline C & 1.916 & 6.009 & -1.398 & C & -0.557 & -7.480 & -0.412 \\
\hline C & 3.245 & 5.957 & -1.028 & C & -1.194 & -6.301 & -0.768 \\
\hline C & 3.888 & 4.744 & -0.712 & C & 6.078 & 5.822 & 0.002 \\
\hline C & -4.986 & -2.652 & -0.313 & C & 7.461 & 5.756 & -0.068 \\
\hline C & -5.750 & -1.449 & -0.132 & C & 8.106 & 4.637 & -0.572 \\
\hline C & -5.059 & -0.187 & 0.136 & C & 7.414 & 3.468 & -0.851 \\
\hline
\end{tabular}




$\begin{array}{rrrrrrrr}\mathrm{C} & 2.384 & -6.421 & 1.698 & \mathrm{H} & -1.464 & 6.962 & 1.645 \\ \mathrm{C} & -2.384 & -6.421 & -1.697 & \mathrm{H} & -0.132 & 4.860 & 1.789 \\ \mathrm{C} & -8.222 & 2.322 & 1.422 & \mathrm{H} & -1.222 & 2.716 & 1.371 \\ \mathrm{C} & -5.486 & 7.078 & -0.610 & \mathrm{H} & -9.180 & 4.661 & 0.718 \\ \mathrm{C} & 8.222 & 2.321 & -1.423 & \mathrm{H} & -8.046 & 6.612 & -0.251 \\ \mathrm{C} & 5.487 & 7.078 & 0.610 & \mathrm{H} & 0.962 & -8.423 & 0.764 \\ \mathrm{~N} & -5.577 & -3.809 & -0.551 & \mathrm{H} & -0.962 & -8.423 & -0.762 \\ \mathrm{~N} & -7.070 & -1.461 & -0.199 & \mathrm{H} & 8.046 & 6.612 & 0.250 \\ \mathrm{C} & -6.925 & -3.833 & -0.621 & \mathrm{H} & 9.180 & 4.661 & -0.719 \\ \mathrm{C} & -7.684 & -2.637 & -0.443 & \mathrm{H} & 2.501 & -5.548 & 2.339 \\ \mathrm{C} & -7.600 & -5.052 & -0.874 & \mathrm{H} & 3.328 & -6.568 & 1.167 \\ \mathrm{C} & -8.967 & -5.076 & -0.946 & \mathrm{H} & 2.242 & -7.292 & 2.340 \\ \mathrm{C} & -9.721 & -3.889 & -0.771 & \mathrm{H} & -2.242 & -7.292 & -2.339 \\ \mathrm{C} & -9.097 & -2.695 & -0.524 & \mathrm{H} & -2.501 & -5.548 & -2.339 \\ \mathrm{~N} & 7.070 & -1.461 & 0.198 & \mathrm{H} & -3.328 & -6.568 & -1.166 \\ \mathrm{C} & 7.684 & -2.637 & 0.442 & \mathrm{H} & -7.638 & 1.697 & 2.097 \\ \mathrm{C} & 6.925 & -3.833 & 0.620 & \mathrm{H} & -8.644 & 1.669 & 0.653 \\ \mathrm{~N} & 5.577 & -3.809 & 0.551 & \mathrm{H} & -9.062 & 2.731 & 1.987 \\ \mathrm{C} & 9.097 & -2.695 & 0.524 & \mathrm{H} & -5.340 & 7.879 & 0.121 \\ \mathrm{C} & 9.721 & -3.889 & 0.770 & \mathrm{H} & -6.176 & 7.463 & -1.363 \\ \mathrm{C} & 8.967 & -5.076 & 0.946 & \mathrm{H} & -4.529 & 6.895 & -1.096 \\ \mathrm{C} & 7.600 & -5.052 & 0.873 & \mathrm{H} & 9.062 & 2.731 & -1.988 \\ \mathrm{H} & -0.946 & -0.501 & 0.345 & \mathrm{H} & 7.637 & 1.697 & -2.098 \\ \mathrm{H} & -3.359 & -4.677 & -0.471 & \mathrm{H} & 8.644 & 1.668 & -0.655 \\ \mathrm{H} & 0.946 & -0.501 & -0.345 & \mathrm{H} & 4.530 & 6.895 & 1.096 \\ \mathrm{H} & 3.359 & -4.677 & 0.471 & \mathrm{H} & 6.177 & 7.463 & 1.363 \\ \mathrm{H} & 1.983 & 1.174 & -0.399 & \mathrm{H} & 5.340 & 7.879 & -0.121 \\ \mathrm{H} & 6.860 & 0.883 & -0.185 & \mathrm{H} & -7.004 & -5.946 & -1.005 \\ \mathrm{H} & 1.221 & 2.716 & -1.370 & \mathrm{H} & -9.483 & -6.008 & -1.140 \\ \mathrm{H} & 0.132 & 4.860 & -1.788 & \mathrm{H} & -10.802 & -3.933 & -0.833 \\ \mathrm{H} & 1.463 & 6.962 & -1.644 & \mathrm{H} & -9.653 & -1.776 & -0.387 \\ \mathrm{H} & 3.809 & 6.874 & -1.037 & \mathrm{H} & 9.653 & -1.776 & 0.386 \\ \mathrm{H} & -6.860 & 0.883 & 0.185 & \mathrm{H} & 10.802 & -3.933 & 0.832 \\ \mathrm{H} & -1.983 & 1.174 & 0.400 & \mathrm{H} & 9.484 & -6.008 & 1.139 \\ \mathrm{H} & -3.809 & 6.874 & 1.038 & \mathrm{H} & 7.004 & -5.946 & 1.005 \\ & & & & & & & \end{array}$

Electronic Energy: -3144.978381

Thermally Corrected Gibbs Free Energy: -3144.059044

Thermally Corrected Enthalpy: -3143.905532

Table S13. Cartesian coordinates of calculated 3-di-g. 


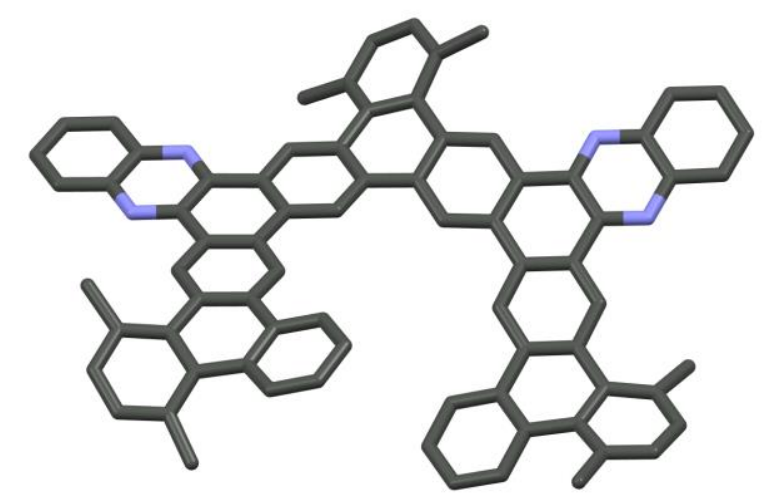

Figure S33. DFT calculated structure of 3-di-x.

$\begin{array}{rrrrrrrr}\text { C } & -0.341 & 5.305 & 7.146 & \text { C } & -1.979 & 9.558 & -3.769 \\ \text { C } & 0.024 & 6.001 & 5.851 & \text { C } & -0.647 & 2.739 & -3.748 \\ \text { C } & -0.152 & 7.376 & 5.810 & \text { C } & -0.435 & 1.360 & -3.792 \\ \text { C } & 0.119 & 8.104 & 4.662 & \text { C } & -0.116 & 0.720 & -2.566 \\ \text { C } & 0.395 & 7.478 & 3.456 & \text { C } & 0.014 & 1.477 & -1.401 \\ \text { C } & 0.435 & 6.059 & 3.430 & \text { C } & -0.362 & 0.616 & -5.062 \\ \text { C } & 0.450 & 5.345 & 4.667 & \text { C } & -0.885 & 1.111 & -6.284 \\ \text { C } & 0.703 & 8.382 & 2.280 & \text { C } & -1.926 & 2.203 & -6.405 \\ \text { C } & 0.989 & 3.975 & 4.661 & \text { C } & -0.467 & 0.512 & -7.464 \\ \text { C } & 1.549 & 3.399 & 5.818 & \text { N } & -0.934 & 6.998 & -1.401 \\ \text { C } & 2.151 & 2.156 & 5.801 & \text { N } & -1.187 & 5.484 & -3.749 \\ \text { C } & 2.234 & 1.440 & 4.609 & \text { C } & 0.341 & -5.305 & 7.146 \\ \text { C } & 1.731 & 1.997 & 3.452 & \text { C } & -0.024 & -6.001 & 5.851 \\ \text { C } & 1.109 & 3.257 & 3.449 & \text { C } & 0.152 & -7.376 & 5.810 \\ \text { C } & 0.632 & 3.869 & 2.214 & \text { C } & -0.119 & -8.104 & 4.662 \\ \text { C } & 0.375 & 5.267 & 2.190 & \text { C } & -0.395 & -7.478 & 3.456 \\ \text { C } & -0.080 & 5.805 & 0.984 & \text { C } & -0.435 & -6.059 & 3.430 \\ \text { C } & -0.247 & 5.049 & -0.173 & \text { C } & -0.450 & -5.345 & 4.667 \\ \text { C } & 0.000 & 3.658 & -0.148 & \text { C } & -0.703 & -8.382 & 2.280 \\ \text { C } & -0.558 & 3.484 & -2.575 & \text { C } & -0.989 & -3.975 & 4.661 \\ \text { C } & -0.819 & 4.924 & -2.610 & \text { C } & -1.549 & -3.399 & 5.818 \\ \text { C } & -0.685 & 5.700 & -1.408 & \text { C } & -2.151 & -2.156 & 5.801 \\ \text { C } & 0.421 & 3.110 & 1.061 & \text { C } & -2.234 & -1.440 & 4.609 \\ \text { C } & -1.596 & 8.967 & -2.595 & \text { C } & -1.731 & -1.997 & 3.452 \\ \text { C } & -1.316 & 7.579 & -2.557 & \text { C } & -1.109 & -3.257 & 3.449 \\ \text { C } & -0.192 & 2.855 & -1.363 & \text { C } & -0.632 & -3.869 & 2.214 \\ \text { C } & -1.440 & 6.809 & -3.753 & \text { C } & -0.375 & -5.267 & 2.190 \\ \text { C } & -1.838 & 7.451 & -4.953 & \text { C } & 0.080 & -5.805 & 0.984 \\ \text { C } & -2.100 & 8.795 & -4.956 & \text { C } & 0.247 & -5.049 & -0.173\end{array}$




\begin{tabular}{|c|c|c|c|c|c|c|c|}
\hline C & 0.000 & -3.658 & -0.148 & $\mathrm{H}$ & 0.576 & 2.044 & 1.124 \\
\hline C & 0.558 & -3.484 & -2.575 & $\mathrm{H}$ & -1.496 & 9.531 & -1.675 \\
\hline C & 0.819 & -4.924 & -2.610 & $\mathrm{H}$ & -1.925 & 6.847 & -5.848 \\
\hline C & 0.685 & -5.700 & -1.408 & $\mathrm{H}$ & -2.404 & 9.285 & -5.873 \\
\hline C & -0.421 & -3.110 & 1.061 & $\mathrm{H}$ & -2.193 & 10.620 & -3.797 \\
\hline C & 1.596 & -8.967 & -2.595 & $\mathrm{H}$ & -0.845 & 3.298 & -4.644 \\
\hline C & 1.316 & -7.579 & -2.557 & $\mathrm{H}$ & 0.331 & 0.969 & -0.503 \\
\hline C & 0.192 & -2.855 & -1.363 & $\mathrm{H}$ & -2.581 & 2.253 & -5.536 \\
\hline C & 1.440 & -6.809 & -3.753 & $\mathrm{H}$ & -2.547 & 2.002 & -7.280 \\
\hline C & 1.838 & -7.451 & -4.953 & $\mathrm{H}$ & -1.490 & 3.196 & -6.546 \\
\hline C & 2.100 & -8.795 & -4.956 & $\mathrm{H}$ & -0.855 & 0.881 & -8.407 \\
\hline C & 1.979 & -9.558 & -3.769 & $\mathrm{H}$ & 1.165 & -5.845 & 7.617 \\
\hline C & 0.647 & -2.739 & -3.748 & $\mathrm{H}$ & -0.479 & -5.290 & 7.869 \\
\hline C & 0.435 & -1.360 & -3.792 & $\mathrm{H}$ & 0.663 & -4.276 & 6.989 \\
\hline C & 0.116 & -0.720 & -2.566 & $\mathrm{H}$ & 0.479 & -7.893 & 6.706 \\
\hline C & -0.014 & -1.477 & -1.401 & $\mathrm{H}$ & -0.081 & -9.187 & 4.696 \\
\hline C & 0.362 & -0.616 & -5.062 & $\mathrm{H}$ & -1.137 & -9.311 & 2.656 \\
\hline C & 0.885 & -1.111 & -6.284 & $\mathrm{H}$ & 0.187 & -8.657 & 1.707 \\
\hline C & 1.926 & -2.203 & -6.405 & $\mathrm{H}$ & -1.415 & -7.937 & 1.587 \\
\hline C & 0.467 & -0.512 & -7.464 & $\mathrm{H}$ & -1.565 & -3.957 & 6.739 \\
\hline$N$ & 0.934 & -6.998 & -1.401 & $\mathrm{H}$ & -2.581 & -1.756 & 6.711 \\
\hline$N$ & 1.187 & -5.484 & -3.749 & $\mathrm{H}$ & -2.716 & -0.471 & 4.581 \\
\hline $\mathrm{H}$ & -1.165 & 5.845 & 7.617 & $\mathrm{H}$ & -1.861 & -1.463 & 2.520 \\
\hline $\mathrm{H}$ & 0.479 & 5.290 & 7.869 & $\mathrm{H}$ & 0.358 & -6.840 & 0.908 \\
\hline $\mathrm{H}$ & -0.663 & 4.276 & 6.989 & $\mathrm{H}$ & -0.576 & -2.044 & 1.124 \\
\hline $\mathrm{H}$ & -0.479 & 7.893 & 6.706 & $\mathrm{H}$ & 1.496 & -9.531 & -1.675 \\
\hline $\mathrm{H}$ & 0.081 & 9.187 & 4.696 & $\mathrm{H}$ & 1.925 & -6.847 & -5.848 \\
\hline $\mathrm{H}$ & 1.137 & 9.311 & 2.656 & $\mathrm{H}$ & 2.404 & -9.285 & -5.873 \\
\hline $\mathrm{H}$ & -0.187 & 8.657 & 1.707 & $\mathrm{H}$ & 2.193 & -10.620 & -3.797 \\
\hline $\mathrm{H}$ & 1.415 & 7.937 & 1.587 & $\mathrm{H}$ & 0.845 & -3.298 & -4.644 \\
\hline $\mathrm{H}$ & 1.565 & 3.957 & 6.739 & $\mathrm{H}$ & -0.331 & -0.969 & -0.503 \\
\hline $\mathrm{H}$ & 2.581 & 1.756 & 6.711 & $\mathrm{H}$ & 2.581 & -2.253 & -5.536 \\
\hline $\mathrm{H}$ & 2.716 & 0.471 & 4.581 & $\mathrm{H}$ & 2.547 & -2.002 & -7.280 \\
\hline $\mathrm{H}$ & 1.861 & 1.463 & 2.520 & $\mathrm{H}$ & 1.490 & -3.196 & -6.546 \\
\hline $\mathrm{H}$ & -0.358 & 6.840 & 0.908 & $\mathrm{H}$ & 0.855 & -0.881 & -8.407 \\
\hline
\end{tabular}

Electronic Energy: -3144.977184

Thermally Corrected Gibbs Free Energy: -3144.070185

Thermally Corrected Enthalpy: -3143.911525

Table S14. Cartesian coordinates of calculated 3-di-x. 


\section{Powder X-ray diffraction (PXRD) data}

To verify substance uniformity, experimental PXRD data of bulk material was compared to predicted PXRD data derived from single-crystal structures. Predicted PXRD spectra were obtained in Mercury using the MicroED structures obtained for the respective helicenes. Experimental diffraction data were collected with $0.02^{\circ}$ steps using a Bruker AXS D8 Advance diffractometer equipped with $\mathrm{Cu}-\mathrm{Ka}$ radiation $\left(\lambda_{\text {avg }}=1.5418 \AA\right.$ ) , a Göbel mirror, a Lynxeye linear position-sensitive director, and mounting the following optics: fixed divergence slit $(0.6 \mathrm{~mm})$, receiving slit $(3 \mathrm{~mm})$, and secondary beam Soller slits $\left(2.5^{\circ}\right)$. The generator was set at $40 \mathrm{kV}$ and $40 \mathrm{~mA}$. Samples were loaded on zero background sample holders for measurement.
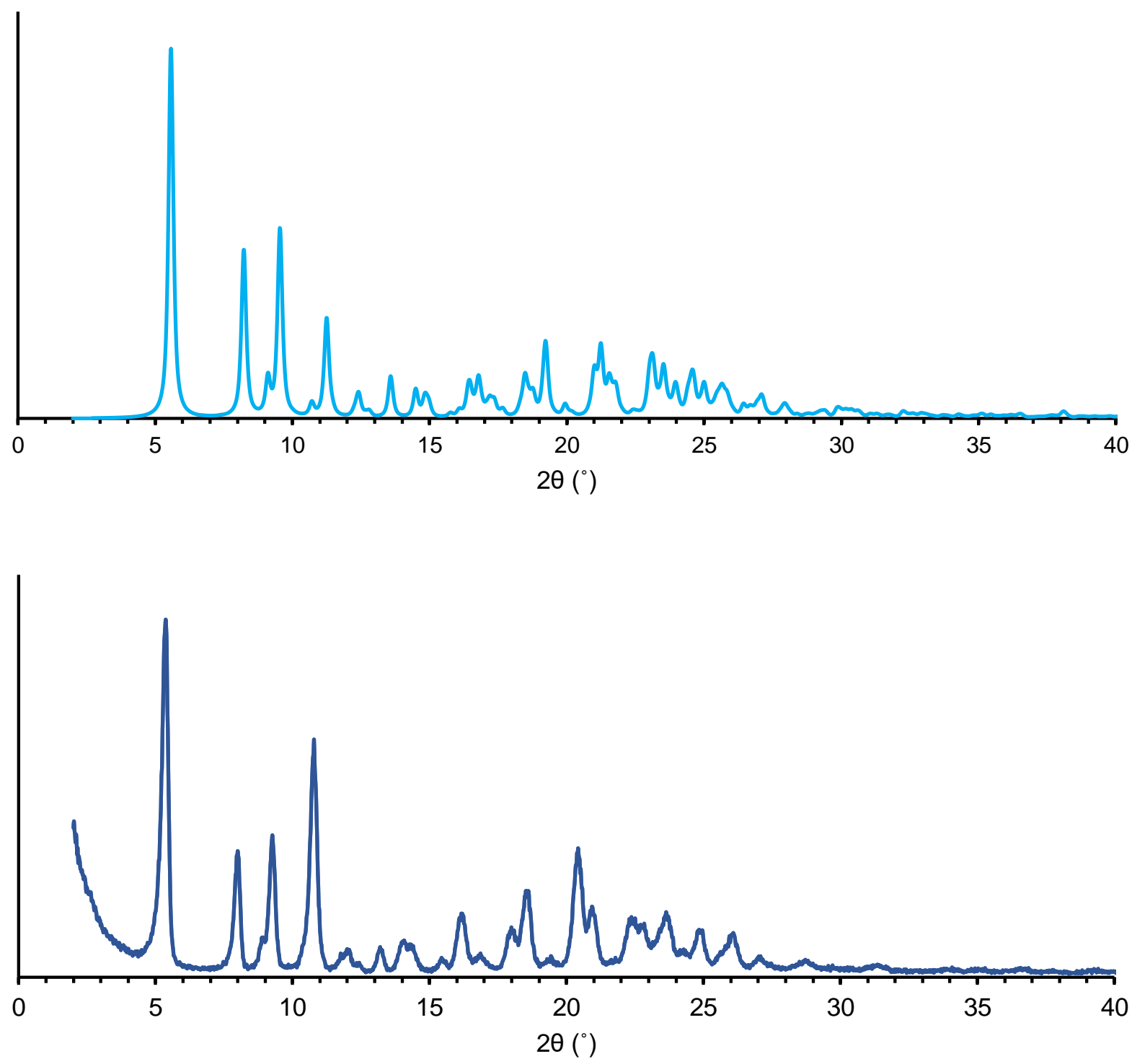

Figure S34. Comparison of the predicted (light blue) and experimental (blue) powder X-ray diffraction patterns for $\mathbf{1 a}$. 

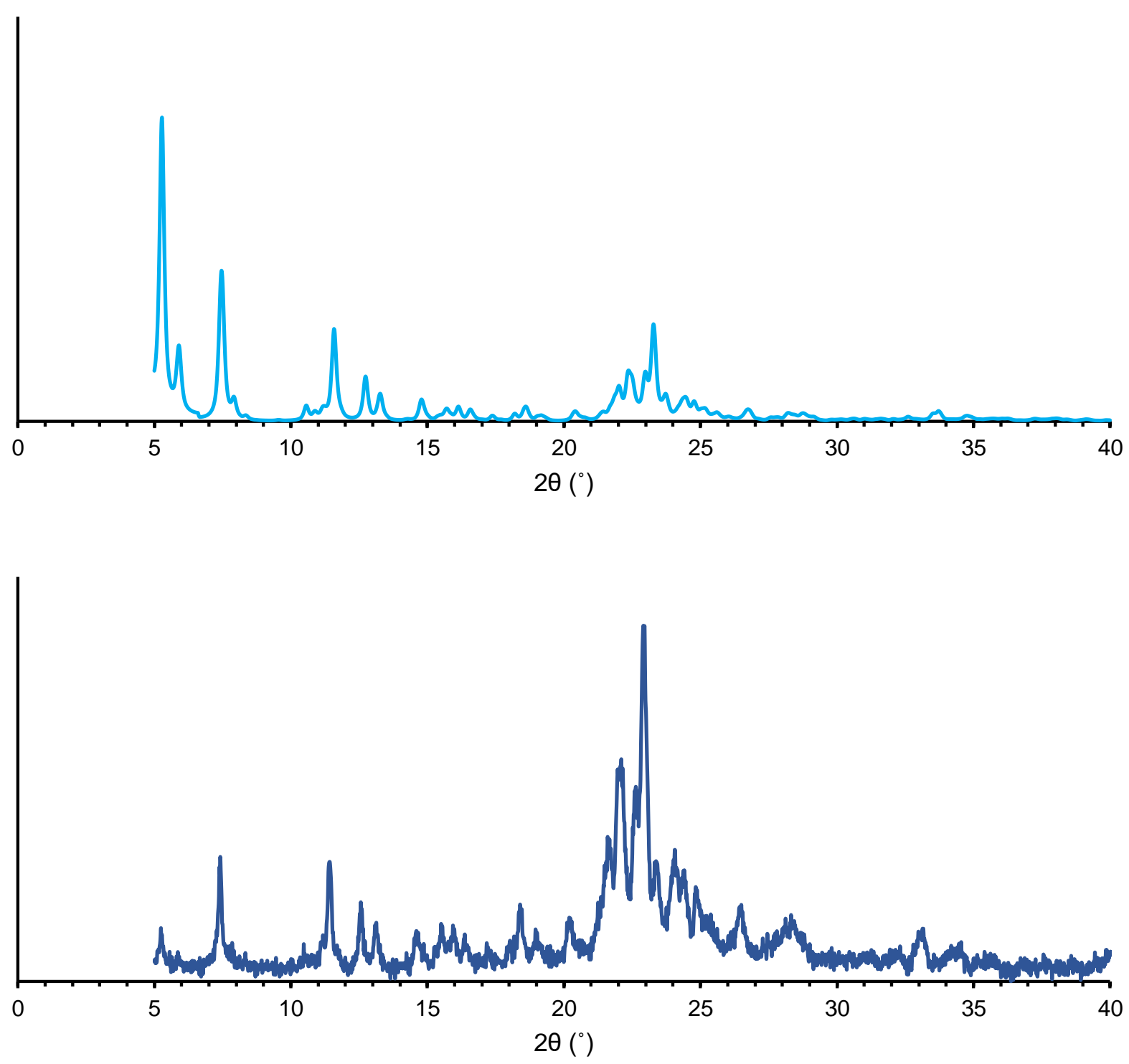

Figure S35. Comparison of the predicted (light blue) and experimental (blue) powder X-ray diffraction patterns for 2-mon. 

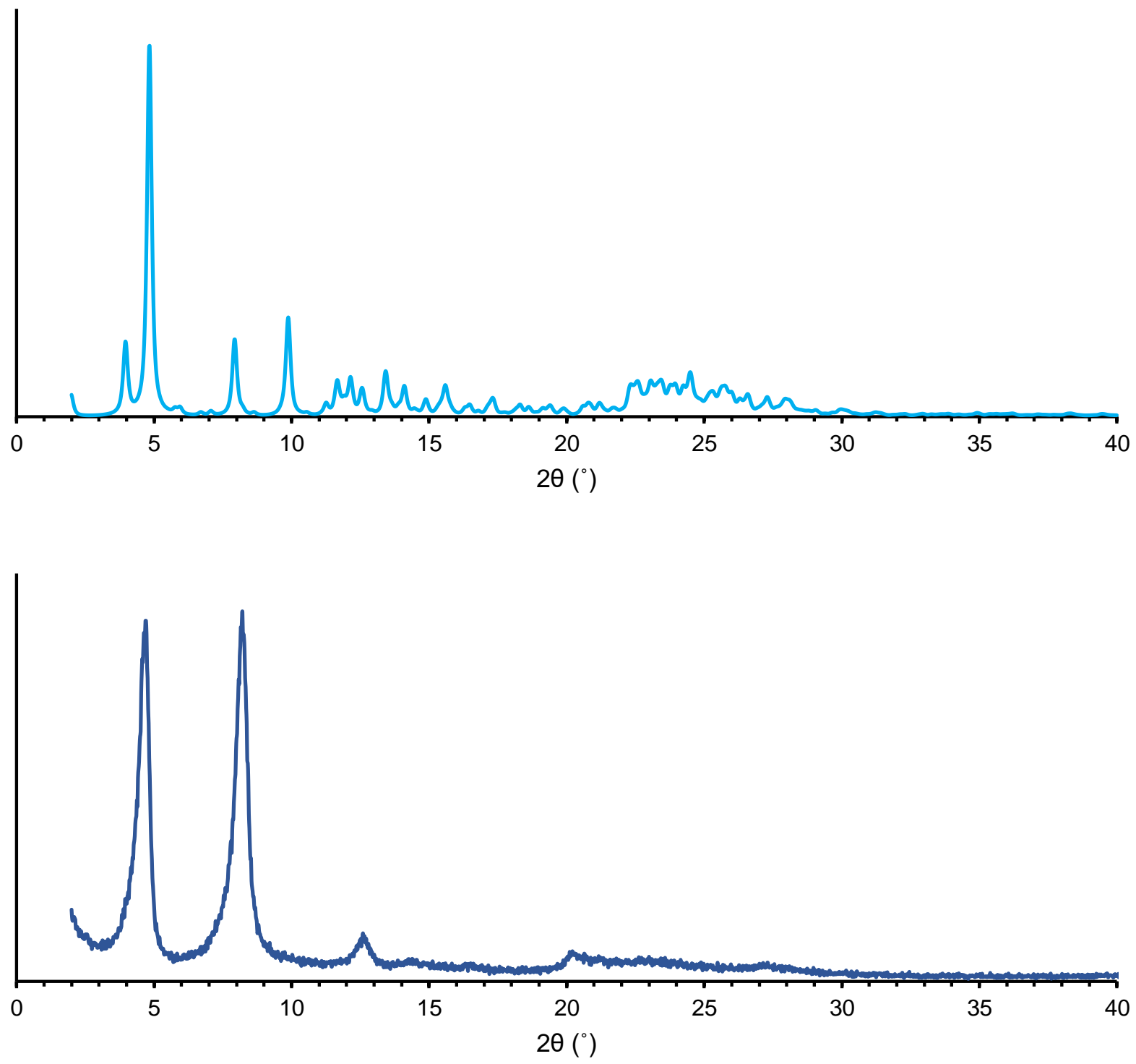

Figure S36. Comparison of the predicted (light blue) and experimental (blue) powder X-ray diffraction patterns for 2-di. We note that the two peaks at low angles line up with those at approximately $5^{\circ}$ and $8^{\circ}$ in the predicted pattern, though it does exhibit poor crystallinity that obscures observation of many other peaks. However, the peaks that are represented are consistent with preferred orientation along the $\left(\begin{array}{lll}0 & 0 & 1\end{array}\right)$ direction, in line with the needle-like crystal habit observed in the microED sample in Figure S14. 

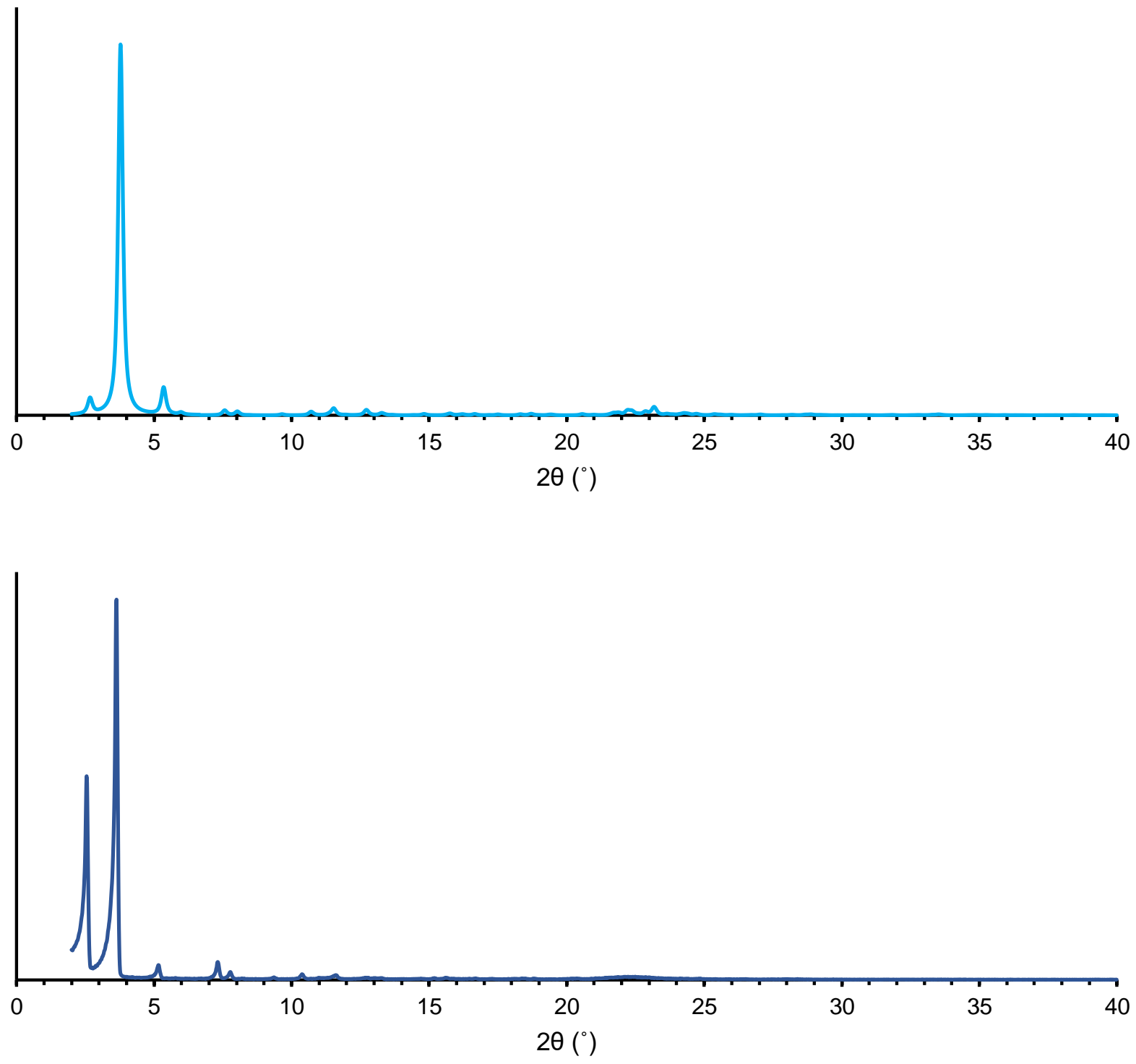

Figure S37. Comparison of the predicted (light blue) and experimental (blue) powder X-ray diffraction patterns for 3-mon. 

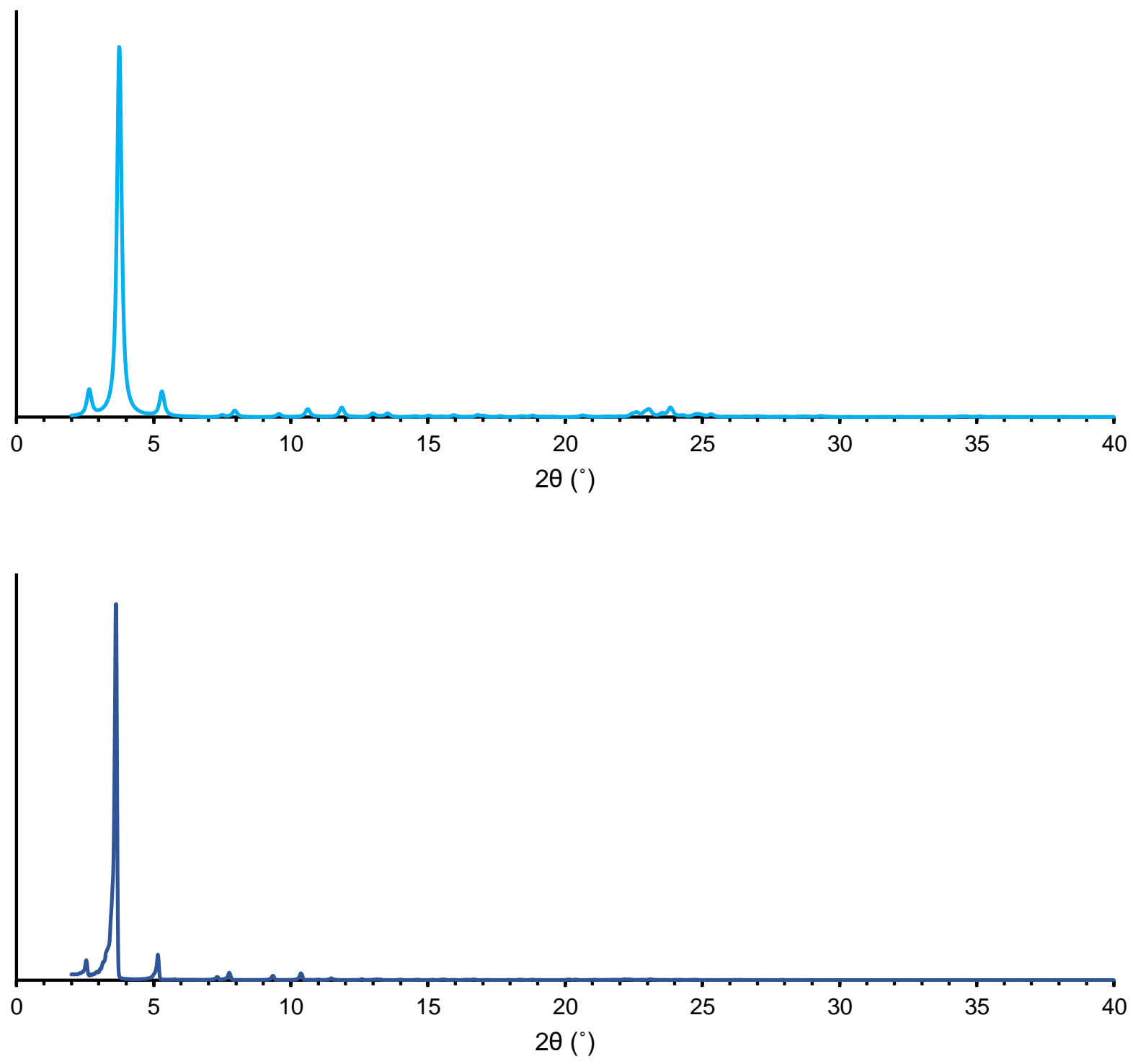

Figure S38. Comparison of the predicted (light blue) and experimental (blue) powder X-ray diffraction patterns for 3-di. 


\section{References}

(1) Armarego, W. L. F.; Chai, C. L. L. Chapter 5 - Purification of Inorganic and Metal-Organic Chemicals: (Including Organic Compounds of B, Bi, P, Se, Si, and Ammonium and Metal Salts of Organic Acids). In Purification of Laboratory Chemicals (Sixth Edition); ButterworthHeinemann: Oxford, 2009; pp 445-576. https://doi.org/10.1016/B978-1-85617-5678.50013-5.

(2) Kiel, G. R.; Patel, S. C.; Smith, P. W.; Levine, D. S.; Tilley, T. D. Expanded Helicenes: A General Synthetic Strategy and Remarkable Supramolecular and Solid-State Behavior. J. Am. Chem. Soc. 2017, 139 (51), 18456-18459. https://doi.org/10.1021/jacs.7b10902.

(3) Nannenga, B. L.; Shi, D.; Leslie, A. G. W.; Gonen, T. High-Resolution Structure Determination by Continuous-Rotation Data Collection in MicroED. Nat. Methods 2014, 11 (9), 927-930. https://doi.org/10.1038/nmeth.3043.

(4) Kabsch, W. Integration, Scaling, Space-Group Assignment and Post-Refinement. Acta $\begin{array}{llllll}\text { Crystallogr. } D & \text { Biol. } & \text { Crystallogr. 2010, } 66 & \text { (2), } & \text { 133-144. }\end{array}$ https://doi.org/10.1107/S0907444909047374.

(5) Sheldrick, G. M. SHELXT - Integrated Space-Group and Crystal-Structure Determination. Acta Crystallogr. Sect. Found. Adv. 2015, 71 (1), 3-8. https://doi.org/10.1107/S2053273314026370.

(6) Schneider, T. R.; Sheldrick, G. M. Substructure Solution with SHELXD. Acta Crystallogr. D Biol. Crystallogr. 2002, 58 (10), 1772-1779. https://doi.org/10.1107/S0907444902011678.

(7) Sheldrick, G. M. Crystal Structure Refinement with SHELXL. Acta Crystallogr. Sect. C Struct. Chem. 2015, 71 (1), 3-8. https://doi.org/10.1107/S2053229614024218.

(8) Hübschle, C. B.; Sheldrick, G. M.; Dittrich, B. ShelXle: A Qt Graphical User Interface for SHELXL. J. Appl. Crystallogr. 2011, 44 (6), 1281-1284. https://doi.org/10.1107/S0021889811043202.

(9) Calio, F.; Alessandro Lazzari. Elements of Mathematics with Numerical Applications, 2nd ed.; Società Editrice Esculapio, 2017.

(10) Enkhbayar, P.; Damdinsuren, S.; Osaki, M.; Matsushima, N. HELFIT: Helix Fitting by a Total Least Squares Method. Comput. Biol. Chem. 2008, 32 (4), 307-310. https://doi.org/10.1016/j.compbiolchem.2008.03.012.

(11) Nakakuki, Y.; Hirose, T.; Matsuda, K. Synthesis of a Helical Analogue of Kekulene: A Flexible $\pi$-Expanded Helicene with Large Helical Diameter Acting as a Soft Molecular Spring. J. Am. Chem. Soc. 2018, $140 \quad$ (45), 15461-15469. https://doi.org/10.1021/jacs.8b09825.

(12) Gaussian 16, Revision C.01, Frisch, M. J.; Trucks, G. W.; Schlegel, H. B.; Scuseria, G. E.; Robb, M. A.; Cheeseman, J. R.; Scalmani, G.; Barone, V.; Petersson, G. A.; Nakatsuji, H.; Li, X.; Caricato, M.; Marenich, A. V.; Bloino, J.; Janesko, B. G.; Gomperts, R.; Mennucci, B.; Hratchian, H. P.; Ortiz, J. V.; Izmaylov, A. F.; Sonnenberg, J. L.; Williams-Young, D.; Ding, F.; Lipparini, F.; Egidi, F.; Goings, J.; Peng, B.; Petrone, A.; Henderson, T.; Ranasinghe, D.; Zakrzewski, V. G.; Gao, J.; Rega, N.; Zheng, G.; Liang, W.; Hada, M.; Ehara, M.; Toyota, K.; Fukuda, R.; Hasegawa, J.; Ishida, M.; Nakajima, T.; Honda, Y.; Kitao, O.; Nakai, H.; Vreven, T.; Throssell, K.; Montgomery, J. A., Jr.; Peralta, J. E.; Ogliaro, F.; Bearpark, M. J.; Heyd, J. J.; Brothers, E. N.; Kudin, K. N.; Staroverov, V. N.; Keith, T. A.; Kobayashi, R.; Normand, J.; Raghavachari, K.; Rendell, A. P.; Burant, J. C.; lyengar, S. S.; Tomasi, J.; Cossi, M.; Millam, J. M.; Klene, M.; Adamo, C.; Cammi, R.; Ochterski, J. W.; Martin, R. L.; Morokuma, K.; Farkas, O.; Foresman, J. B.; Fox, D. J. Gaussian, Inc., Wallingford CT, 2016. 